\title{
Total Synthesis of Naphterpin and Marinone Natural Products
}

Lauren A. M. Murray, Thomas Fallon, Christopher J. Sumby and Jonathan H. George*

Department of Chemistry, University of Adelaide, Adelaide, SA 5005, Australia.

\section{Supporting Information}

\section{Table of Contents}

1. General methods 2

2. Experimental procedures 3

3. NMR spectra 20

4. Tables of NMR data 62

5. Molecular modelling and NMR calculations 66

6. Single crystal X-ray data $\quad 71$ 


\section{General methods}

All chemicals used were purchased from commercial suppliers and used as received. All reactions were performed under an inert atmosphere of $\mathrm{N}_{2}$. All organic extracts were dried over anhydrous magnesium sulfate. Thin layer chromatography was performed using aluminium sheets coated with silica gel $F_{254}$. Visualization was aided by viewing under a UV lamp and staining with ceric ammonium molybdate followed by heating. All $\mathrm{R}_{\mathrm{f}}$ values were measured to the nearest 0.05 . Flash column chromatography was performed using 40-63 micron grade silica gel. Melting points were recorded on a Reichart Thermovar Kofler microscope apparatus and are uncorrected. Infrared spectra were recorded using an FT-IR spectrometer as the neat compounds. High field NMR spectra were recorded using both a $500 \mathrm{MHz}$ spectrometer $\left({ }^{1} \mathrm{H}\right.$ at $500 \mathrm{MHz},{ }^{13} \mathrm{C}$ at $125 \mathrm{MHz}$ ) and $600 \mathrm{MHz}$ spectrometer $\left({ }^{1} \mathrm{H}\right.$ at $600 \mathrm{MHz},{ }^{13} \mathrm{C}$ at $\left.150 \mathrm{MHz}\right)$ as indicated. The solvent used for spectra was $\mathrm{CDCl}_{3}$ unless otherwise specified. ${ }^{1} \mathrm{H}$ chemical shifts are reported in ppm on the $\delta$-scale relative to TMS $(\delta$ $0.0)$ and ${ }^{13} \mathrm{C}$ NMR are reported in ppm relative to $\mathrm{CDCl}_{3}(\delta 77.16)$. Multiplicities are reported as (br) broad, (s) singlet, (d) doublet, (t) triplet, (q) quartet, (quin) quintet, (sext) sextet, (hept) heptet and (m) multiplet. All $J$-values were rounded to the nearest $0.1 \mathrm{~Hz}$. ESI high-resolution mass spectra were recorded on an ESI-TOF mass spectrometer. 


\section{Experimental procedures}

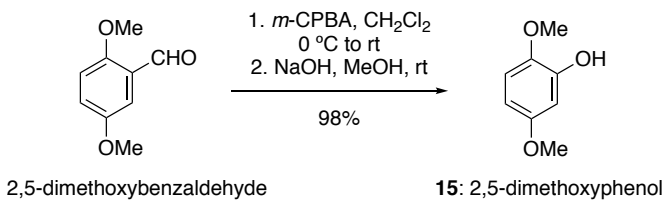

A solution of 2,5-dimethoxybenzaldehyde $(5.00 \mathrm{~g}, 30.1 \mathrm{mmol})$ in $\mathrm{CH}_{2} \mathrm{Cl}_{2}(10 \mathrm{~mL})$ was added dropwise over 15 minutes to a solution of $m$-CPBA $(7.00 \mathrm{~g}, 31.6 \mathrm{mmol})$ in $\mathrm{CH}_{2} \mathrm{Cl}_{2}(40 \mathrm{~mL})$ at $0{ }^{\circ} \mathrm{C}$. The mixture was then warmed to room temperature and stirred for $16 \mathrm{~h}$. The reaction mixture was quenched with saturated $\mathrm{NaHCO}_{3}$ solution $(100 \mathrm{~mL})$. The organic layer was separated, and washed with saturated $\mathrm{NaHCO}_{3}$ solution $(4 \times 100 \mathrm{~mL})$ and saturated $\mathrm{Na}_{2} \mathrm{~S}_{2} \mathrm{O}_{3}$ solution $(100 \mathrm{~mL})$. The organic layer was then dried over anhydrous $\mathrm{MgSO}_{4}$, filtered and concentrated in vacuo. The resulting yellow residue was dissolved in $\mathrm{MeOH}$ and stirred with $10 \% \mathrm{NaOH}(60 \mathrm{~mL})$ for $3 \mathrm{~h}$. The mixture was acidified to $\mathrm{pH} 1$ with $6 \mathrm{M}$ hydrochloric acid and extracted with dichloromethane $(3 \times 50 \mathrm{~mL})$. The combined organic layers were dried over anhydrous $\mathrm{MgSO}_{4}$, filtered and concentrated in vacuo. The residue was purified by flash chromatography (petrol/EtOAc 2:1) to yield 15 (4.56 g, 98\%) as a yellow oil. Data for $\mathbf{1 5}$ matched that previously obtained in the literature. ${ }^{1}$

\section{Data for 15:}

$\mathbf{R}_{\mathbf{f}}=0.30\left(\mathrm{CH}_{2} \mathrm{Cl}_{2}\right)$

IR (neat): $\mathrm{cm}^{-1} 3429,3001,2938,2837,1597,1506,1465,1440,1282 \mathrm{~cm}^{-1}$.

${ }^{1}$ H NMR (500 MHz, CDCl $): 6.76(\mathrm{~d}, J=8.8 \mathrm{~Hz}, 1 \mathrm{H}), 6.56(\mathrm{~d}, J=2.9 \mathrm{~Hz}, 1 \mathrm{H}), 6.37(\mathrm{dd}, J=8.8$, $2.9 \mathrm{~Hz}, 1 \mathrm{H}), 5.76(\mathrm{~s}, 1 \mathrm{H}), 3.82(\mathrm{~s}, 3 \mathrm{H}), 3.73(\mathrm{~s}, 3 \mathrm{H})$.

${ }^{13}$ C NMR (125 MHz, CDCl $): \delta 154.7,146.6,141.1,111.7,104.4,101.9,56.7,55.7$.

${ }^{1}$ Wriede, U.; Fernandez, M.; West, K. F.; Harcourt, D.; Moore, H. W. J. Org. Chem. 1987, 52, 4485. 


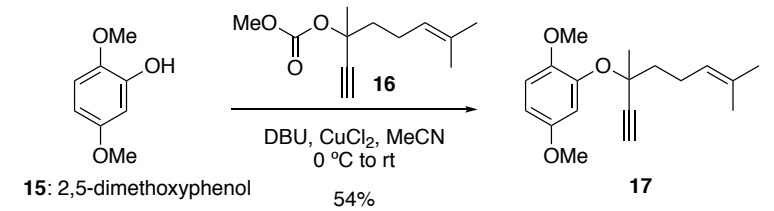

To a solution of $15(250 \mathrm{mg}, 1.62 \mathrm{mmol})$ in $\mathrm{MeCN}(50 \mathrm{~mL})$ at $0{ }^{\circ} \mathrm{C}$ was added DBU $(0.32 \mathrm{~mL}, 2.11$ $\mathrm{mmol})$ dropwise, $\mathrm{CuCl}_{2}(2 \mathrm{mg}, 0.016 \mathrm{mmol})$ and $\mathbf{1 6}^{2}(410 \mathrm{mg}, 1.95 \mathrm{mmol})$. The reaction mixture was stirred at $0{ }^{\circ} \mathrm{C}$ for $8 \mathrm{~h}$ prior to being warmed to $\mathrm{rt}$ and stirred for a further $16 \mathrm{~h}$. The mixture was quenched with saturated $\mathrm{NH}_{4} \mathrm{Cl}$ solution $(50 \mathrm{~mL})$ and extracted with EtOAc $(3 \times 40 \mathrm{~mL})$. The combined organic extracts were washed with saturated brine $(100 \mathrm{~mL})$, dried over anhydrous $\mathrm{MgSO}_{4}$, filtered and concentrated in vacuo. The residue was purified by flash chromatography on $\mathrm{SiO}_{2}(9: 1$ $\rightarrow 4: 1$, petrol/EtOAc gradient elution) to give 17 (252 $\mathrm{mg}, 54 \%)$ as a colourless oil.

\section{Data for 17:}

$\mathbf{R}_{\mathbf{f}}=0.55(4: 1$ petrol/EtOAc)

IR (neat): 3284, 2934, 2834, 1609, 1589, 1503, 1442, $1260 \mathrm{~cm}^{-1}$.

${ }^{1} \mathbf{H}$ NMR (500 MHz, CDCl $): \delta 7.10(\mathrm{~d}, J=3.0 \mathrm{~Hz}, 1 \mathrm{H}), 6.81(\mathrm{~d}, J=8.8 \mathrm{~Hz}, 1 \mathrm{H}), 6.56(\mathrm{dd}, J=8.9$, $3.0 \mathrm{~Hz}, 1 \mathrm{H}), 5.16(\mathrm{t}, J=7.2,1 \mathrm{H}), 3.77(\mathrm{~s}, 3 \mathrm{H}), 3.76(\mathrm{~s}, 3 \mathrm{H}), 2.57(\mathrm{~s}, 1 \mathrm{H}), 2.38-2.22(\mathrm{~m}, 2 \mathrm{H}), 1.99$ (ddd, $J=13.4,11.4,5.2 \mathrm{~Hz}, 1 \mathrm{H}), 1.87$ (ddd, $J=13.4,11.7,5.3 \mathrm{~Hz}, 1 \mathrm{H}), 1.69$ (s, 3H), 1.64 (s, 3H), $1.58(\mathrm{~s}, 3 \mathrm{H})$.

${ }^{13}$ C NMR (125 MHz, $\left.\mathbf{C D C l}_{3}\right): \delta 153.7,147.4,145.8,132.1,123.9,113.6,110.3,107.9,85.6,77.1$, $74.9,56.9,55.8,42.5,26.7,25.8,23.4,17.8$.

HRMS (ESI): calculated for $\mathrm{C}_{18} \mathrm{H}_{24} \mathrm{NaO}_{3} 311.1623[\mathrm{M}+\mathrm{Na}]^{+}$, found 311.1623 .

${ }^{2}$ Prepared according to: (a) Tsuji, J.; Sugiura, T.; Minami, I. Synthesis 1987, 603. (b) Gassner, C.; Hesse, R.; Schmidt, A. W.; Knolker, H.-J. Org. Biomol. Chem. 2014, 12, 6490. 


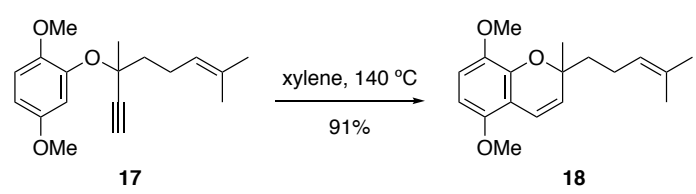

A solution of $17(4.30 \mathrm{~g}, 14.91 \mathrm{mmol})$ in xylene $(300 \mathrm{~mL})$ was stirred at $140^{\circ} \mathrm{C}$ for $3 \mathrm{~h}$. The mixture was cooled to room temperature and concentrated in vacuo. The residue was purified by flash chromatography on $\mathrm{SiO}_{2}(9: 1$, petrol/EtOAc) to give $\mathbf{1 8}(3.90 \mathrm{~g}, 91 \%)$ as a yellow oil.

\section{Data for 18:}

$\mathbf{R}_{\mathbf{f}}=0.55(4: 1 \mathrm{petrol} /$ EtOAc $)$

IR (neat): 2967, 2836, 1491, 1464, 1439, $1386 \mathrm{~cm}^{-1}$.

${ }^{1}$ H NMR (500 MHz, CDCl $): \delta 6.69(\mathrm{t}, J=9.5 \mathrm{~Hz}, 2 \mathrm{H}), 6.29(\mathrm{~d}, J=8.8 \mathrm{~Hz}, 1 \mathrm{H}), 5.55(\mathrm{~d}, J=10.1$ $\mathrm{Hz}, 1 \mathrm{H}), 5.09$ (t, $J=7.1 \mathrm{~Hz}, 1 \mathrm{H}), 3.80$ (s, 3H), 3.77 (s, 3H), 2.12 (q, $J=7.9 \mathrm{~Hz}, 2 \mathrm{H}), 1.86-1.77$ (m, 1H), 1.71 - 1.63 (overlapping m, 1H), 1.65 (s, 3H), 1.56 (s, 3H), 1.43 (s, 3H).

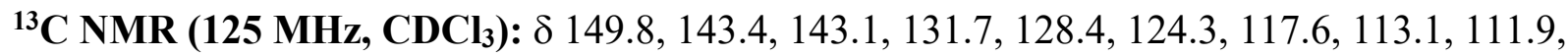
$101.7,78.4,57.3,56.0,40.9,26.3,25.8,22.9,17.7$.

HRMS (ESI): calculated for $\mathrm{C}_{18} \mathrm{H}_{24} \mathrm{NaO}_{3} 311.1623[\mathrm{M}+\mathrm{Na}]^{+}$, found 311.1623. 


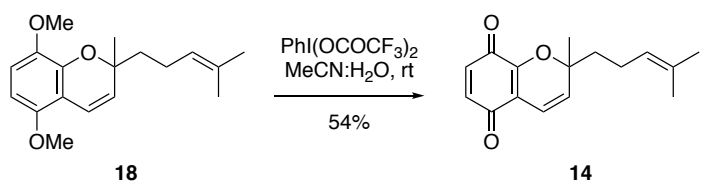

To a solution of $18(2.09 \mathrm{~g}, 7.25 \mathrm{mmol})$ in $\mathrm{MeCN}: \mathrm{H}_{2} \mathrm{O}(120 \mathrm{~mL}, 2: 1 \mathrm{v} / \mathrm{v})$ at room temperature was added $\mathrm{PhI}\left(\mathrm{OCOCF}_{3}\right)_{2}(6.86 \mathrm{~g}, 15.9 \mathrm{mmol})$. The reaction mixture was stirred at room temperature for $5 \mathrm{~min}$. The mixture was quenched with saturated $\mathrm{NaHCO}_{3}$ solution $(50 \mathrm{~mL})$ and extracted with EtOAc $(3 \times 100 \mathrm{~mL})$. The combined organic extracts were washed with $\mathrm{H}_{2} \mathrm{O}(100 \mathrm{~mL})$ and saturated brine (100 mL), dried over anhydrous $\mathrm{MgSO}_{4}$, filtered and concentrated in vacuo. The residue was purified by flash chromatography on $\mathrm{SiO}_{2}(20: 1 \rightarrow 9: 1$, petrol/EtOAc gradient elution) to give $14(1.01 \mathrm{~g}$, $54 \%)$ as a dark red oil.

\section{Data for 14:}

$\mathbf{R}_{\mathbf{f}}=0.40(4: 1 \mathrm{petrol} / \mathrm{EtOAc})$

IR (neat): $\mathrm{cm}^{-1}$ 2971, 2926, 1673, 1646, 1608, 1575, 1448, 1413, 1376, 1335, $1312 \mathrm{~cm}^{-1}$.

${ }^{1}$ H NMR (500 MHz, CDCl $): \delta 6.63(\mathrm{~s}, 2 \mathrm{H}), 6.47(\mathrm{~d}, J=10.0 \mathrm{~Hz}, 1 \mathrm{H}), 5.58(\mathrm{~d}, J=10.1 \mathrm{~Hz}, 1 \mathrm{H})$, $5.07(\mathrm{t}, J=7.1 \mathrm{~Hz}, 1 \mathrm{H}), 2.09(\mathrm{~m}, 2 \mathrm{H}), 1.96-1.87$ (m, 1H), $1.69-1.62$ (overlapping $\mathrm{m}, 1 \mathrm{H}), 1.65$ $(\mathrm{s}, 3 \mathrm{H}), 1.56(\mathrm{~s}, 3 \mathrm{H}), 1.48(\mathrm{~s}, 3 \mathrm{H})$.

${ }^{13}$ C NMR (125 MHz, $\left.\mathbf{C D C l}_{3}\right): \delta 184.4,181.6,150.9,135.9,134.7,132.4,129.3,123.5,115.7$, $115.4,83.3,41.6,27.6,25.7,22.7,17.8$.

HRMS (ESI): calculated for $\mathrm{C}_{16} \mathrm{H}_{19} \mathrm{O}_{3} 259.1334[\mathrm{M}+\mathrm{H}]^{+}$, found 259.1331 . 


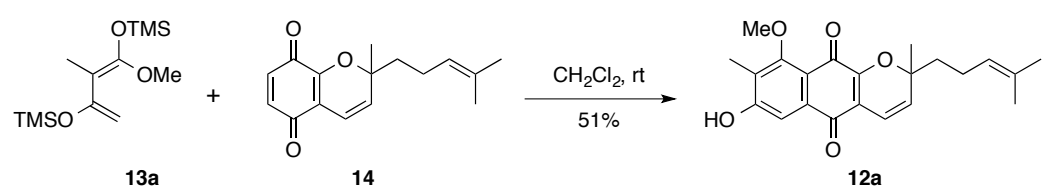

To a solution of $\mathbf{1 4}(916 \mathrm{mg}, 3.55 \mathrm{mmol})$ in $\mathrm{CH}_{2} \mathrm{Cl}_{2}(20 \mathrm{~mL})$ at room temperature was added $\mathbf{1 3 a}^{3}$ $(1.27 \mathrm{~g}, 4.61 \mathrm{mmol})$. The reaction mixture was stirred at room temperature for $2 \mathrm{~h}$. A second portion of 13a (681 mg, $2.48 \mathrm{mmol})$ was then added and the reaction was stirred at room temperature for a further $30 \mathrm{~min}$. The mixture was quenched with $1 \mathrm{M} \mathrm{HCl}(20 \mathrm{~mL})$ and stirred at room temperature for $30 \mathrm{~min}$. The mixture was extracted with EtOAc $(3 \times 40 \mathrm{~mL})$. The combined organic extracts were washed with saturated brine $(150 \mathrm{~mL})$, dried over anhydrous $\mathrm{MgSO}_{4}$, filtered and concentrated in vacuo. The residue was purified by flash chromatography on $\mathrm{SiO}_{2}(20: 1 \rightarrow 9: 1$, petrol/EtOAc gradient elution) to give 12a (667 $\mathrm{mg}, 51 \%)$ as a dark orange solid.

\section{Data for 12a:}

$\mathbf{R}_{\mathbf{f}}=0.20(4: 1 \mathrm{petrol} / \mathrm{EtOAc})$

$\mathbf{M p}=132-134^{\circ} \mathrm{C}$

IR (neat): 1362, 2928, 1625, 1573, 1436, 1332, 1281, $1248 \mathrm{~cm}^{-1}$.

${ }^{1}$ H NMR (500 MHz, CDCl$): \delta 8.65(\mathrm{~s}, 1 \mathrm{H}), 7.72(\mathrm{~s}, 1 \mathrm{H}), 6.60(\mathrm{~d}, J=10.1 \mathrm{~Hz}, 1 \mathrm{H}), 5.60(\mathrm{~d}, J=$ $10.1 \mathrm{~Hz}, 1 \mathrm{H}), 5.05(\mathrm{t}, J=7.0 \mathrm{~Hz}, 1 \mathrm{H}), 3.89(\mathrm{~s}, 3 \mathrm{H}), 2.25$ (s, 3H), $2.15-2.07(\mathrm{~m}, 2 \mathrm{H}), 1.96-1.89$ (m, 1H), $1.72-1.64(\mathrm{~m}, 1 \mathrm{H}), 1.62(\mathrm{~s}, 3 \mathrm{H}), 1.54(\mathrm{~s}, 3 \mathrm{H}), 1.50(\mathrm{~s}, 3 \mathrm{H})$.

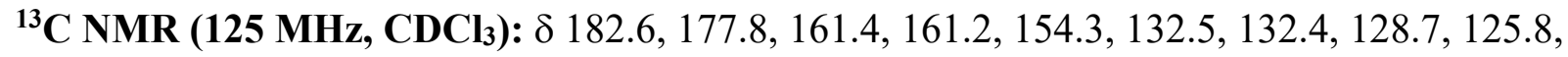
$123.5,116.8,115.7,115.5,110.6,83.8,61.3,41.7,27.7,25.7,22.8,17.8,9.0$.

HRMS (ESI): calculated for $\mathrm{C}_{22} \mathrm{H}_{25} \mathrm{O}_{5} 369.1702[\mathrm{M}+\mathrm{H}]^{+}$, found 369.1700 .

\footnotetext{
${ }^{3}$ Prepared according to: Nawrat, C. C.; Lewis, W.; Moody, C. J. J. Org. Chem. 2011, 76, 7872.
} 


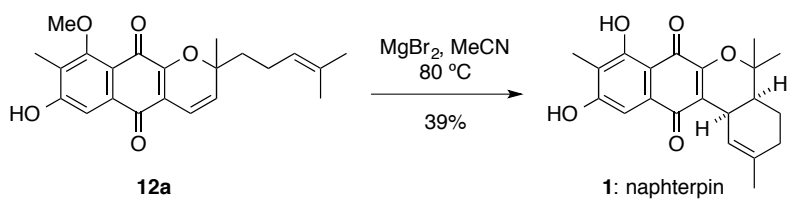

To a solution of $12 \mathrm{a}(500 \mathrm{mg}, 1.36 \mathrm{mmol})$ in $\mathrm{MeCN}(40 \mathrm{~mL})$ at room temperature was added $\mathrm{MgBr}_{2}$ $(1.25 \mathrm{~g}, 6.79 \mathrm{mmol})$. The reaction was stirred at $80^{\circ} \mathrm{C}$ for $16 \mathrm{~h}$. The mixture was cooled to room temperature, quenched with $1 \mathrm{M} \mathrm{HCl}(50 \mathrm{~mL})$ and extracted with EtOAc $(3 \times 50 \mathrm{~mL})$. The combined organic extracts were washed with saturated brine $(100 \mathrm{~mL})$, dried over anhydrous $\mathrm{MgSO}_{4}$, filtered and concentrated in vacuo. The residue was purified by flash chromatography on $\mathrm{SiO}_{2}(9: 1 \rightarrow 4: 1$, petrol/EtOAc gradient elution) to give crude $\mathbf{1}$ as a brown oil. Trituration from $\mathrm{CH}_{2} \mathrm{Cl}_{2}$ gave pure $\mathbf{1}$ (190 mg, 39\%) as an orange amorphous solid.

\section{Data for 1:}

$\mathbf{R}_{\mathbf{f}}=0.60(1: 1 \mathrm{petrol} / \mathrm{EtOAc})$

IR (neat): 1362, 2928, 1625, 1573, 1436, 1332, 1281, $1248 \mathrm{~cm}^{-1}$.

${ }^{1}$ H NMR (500 MHz, CDCl $): \delta 12.29(\mathrm{~s}, 1 \mathrm{H}), 7.18(\mathrm{~s}, 1 \mathrm{H}), 6.45(\mathrm{~s}, 1 \mathrm{H}), 6.06(\mathrm{~d}, J=5.2 \mathrm{~Hz}, 1 \mathrm{H})$, $3.51-3.46(\mathrm{~m}, 1 \mathrm{H}), 2.18(\mathrm{~s}, 3 \mathrm{H}), 2.08-1.91(\mathrm{~m}, 3 \mathrm{H}), 1.77(\mathrm{ddd}, J=12.1,6.2,2.8 \mathrm{~Hz}, 1 \mathrm{H}), 1.68$ $(\mathrm{s}, 3 \mathrm{H}), 1.56(\mathrm{~s}, 3 \mathrm{H}), 1.34(\mathrm{~s}, 3 \mathrm{H}), 1.34-1.27(\mathrm{~m}, 1 \mathrm{H})$.

${ }^{13}$ C NMR (125 MHz, CDCl 3$): \delta 184.2,183.5,162.5,160.7,153.2,136.2,131.8,123.7,120.3$, $116.8,108.5,107.9,80.7,39.9,31.3,29.8,25.8,25.2,23.7,20.5,7.9$.

HRMS (ESI): calculated for $\mathrm{C}_{21} \mathrm{H}_{23} \mathrm{O}_{5} 355.1545[\mathrm{M}+\mathrm{H}]^{+}$, found 355.1543 . 

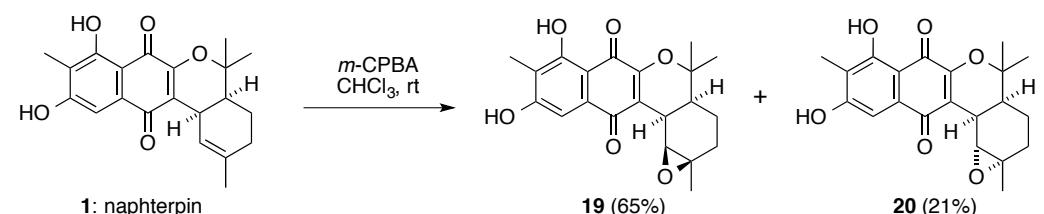

To a solution of $1(75 \mathrm{mg}, 0.21 \mathrm{mmol})$ in $\mathrm{CHCl}_{3}(8 \mathrm{~mL})$ at room temperature was added $m$-CPBA $(77 \%, 57 \mathrm{mg}, 0.26 \mathrm{mmol})$. The reaction was stirred at room temperature for $1 \mathrm{~h}$. The mixture was diluted with saturated $\mathrm{NaHCO}_{3}$ solution $(15 \mathrm{~mL})$ and extracted with $\mathrm{CHCl}_{3}(3 \times 10 \mathrm{~mL})$. The combined organic extracts were washed with saturated $\mathrm{Na}_{2} \mathrm{~S}_{2} \mathrm{O}_{3}$ solution $(40 \mathrm{~mL})$ and saturated brine $(40 \mathrm{~mL})$, dried over anhydrous $\mathrm{MgSO}_{4}$, filtered and concentrated in vacuo. The residue was purified by flash chromatography on $\mathrm{SiO}_{2}(4: 1 \rightarrow 1: 1$, petrol/EtOAc gradient elution) to give $19(51 \mathrm{mg}, 65 \%)$ as a fine yellow powder and $\mathbf{2 0}(16 \mathrm{mg}, 21 \%)$ as a fine orange powder.

\section{Data for 19:}

$\mathbf{R}_{\mathbf{f}}=0.30(1: 1 \mathrm{petrol} / \mathrm{EtOAc})$

IR (neat): 3276, 2963, 2924, 2854, 1633, 1591, 1434, 1377, 1325, $1289 \mathrm{~cm}^{-1}$.

${ }^{1}$ H NMR (600 MHz, CDCl $): \delta 12.16(\mathrm{~s}, 1 \mathrm{H}), 8.42(\mathrm{~s}, 1 \mathrm{H}), 6.89(\mathrm{~s}, 1 \mathrm{H}), 4.17(\mathrm{~d}, J=5.5 \mathrm{~Hz}, 1 \mathrm{H})$, $3.51(\mathrm{t}, J=6.2 \mathrm{~Hz}, 1 \mathrm{H}), 2.18-2.14(\mathrm{~m}, 1 \mathrm{H}), 1.95(\mathrm{~s}, 3 \mathrm{H}), 1.78(\mathrm{ddd}, J=14.9,12.9,5.0 \mathrm{~Hz}, 1 \mathrm{H})$, $1.69-1.63(\mathrm{~m}, 1 \mathrm{H}), 1.58(\mathrm{ddd}, J=12.9,7.1,2.6 \mathrm{~Hz}, 1 \mathrm{H}), 1.53(\mathrm{~s}, 3 \mathrm{H}), 1.45(\mathrm{~s}, 3 \mathrm{H}), 1.33-1.27(\mathrm{~m}$, $1 \mathrm{H}), 1.30(\mathrm{~s}, 3 \mathrm{H})$.

${ }^{13}$ C NMR (150 MHz, CDCl $)$ ): $\delta$ 183.9, 182.4, 162.5, 161.7, 155.0, 130.9, 120.0, 117.3, 108.5, 107.8, $79.6,62.2,62.1,39.2,30.2,30.1,26.2,24.7,22.9,17.5,7.7$.

HRMS (ESI): calculated for $\mathrm{C}_{21} \mathrm{H}_{23} \mathrm{O}_{6} 371.1495[\mathrm{M}+\mathrm{H}]^{+}$, found 371.1492.

\section{Data for 20:}

$\mathbf{R}_{\mathbf{f}}=0.20(1: 1 \mathrm{petrol} / \mathrm{EtOAc})$

IR (neat): 3346, 2940, 1629, 1587, 1564, 1435, 1330, $1249 \mathrm{~cm}^{-1}$.

${ }^{1}$ H NMR (600 MHz, CDCl $): \delta 12.22(\mathrm{~s}, 1 \mathrm{H}), 7.61(\mathrm{~s}, 1 \mathrm{H}), 7.22(\mathrm{~s}, 1 \mathrm{H}), 3.84(\mathrm{~s}, 1 \mathrm{H}), 3.47$ (dd, $J=$ 5.9, $1.9 \mathrm{~Hz}, 1 \mathrm{H}), 2.16(\mathrm{~s}, 3 \mathrm{H}), 1.99-1.92(\mathrm{~m}, 1 \mathrm{H}), 1.89(\mathrm{td}, J=6.0,3.0 \mathrm{~Hz}, 1 \mathrm{H}), 1.86-1.79$ (m, $1 \mathrm{H}), 1.76-1.70(\mathrm{~m}, 1 \mathrm{H}), 1.46(\mathrm{~s}, 3 \mathrm{H}), 1.41(\mathrm{~s}, 3 \mathrm{H}), 1.34-1.26$ (overlapping m, $1 \mathrm{H}), 1.30$ (s, 3H).

${ }^{13}$ C NMR (150 MHz, $\left.\mathbf{C D C l}_{3}\right): \delta 184.2,182.7,162.8,161.6,155.7,131.4,119.8,117.4,108.4$, $108.3,81.4,61.3,59.2,36.3,32.0,27.1,25.8,25.1,23.8,19.1,8.0$.

HRMS (ESI): calculated for $\mathrm{C}_{21} \mathrm{H}_{23} \mathrm{O}_{6} 371.1495[\mathrm{M}+\mathrm{H}]^{+}$, found 371.1492 . 


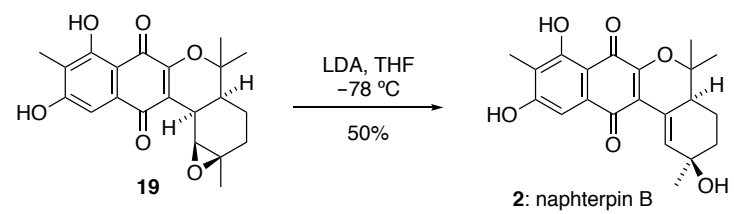

To a solution of diisopropylamine $(0.05 \mathrm{~mL}, 0.38 \mathrm{mmol})$ in THF $(2 \mathrm{~mL})$ at $0{ }^{\circ} \mathrm{C}$ was added $n$-BuLi (1.6 $\mathrm{M}$ in cyclohexane, $0.24 \mathrm{~mL}, 0.38 \mathrm{mmol}$ ). The reaction was cooled to $-78^{\circ} \mathrm{C}$ and a solution of 19 $(14 \mathrm{mg}, 0.04 \mathrm{mmol})$ in THF $(1 \mathrm{~mL})$ was added dropwise to the reaction. The reaction was stirred at $-78^{\circ} \mathrm{C}$ for $1.5 \mathrm{~h}$. The mixture was quenched with saturated $\mathrm{NH}_{4} \mathrm{Cl}$ solution $(5 \mathrm{~mL})$ and extracted with EtOAc $(3 \times 5 \mathrm{~mL})$. The combined organic extracts were washed with saturated brine $(15 \mathrm{~mL})$, dried over anhydrous $\mathrm{MgSO}_{4}$, filtered and concentrated in vacuo. The residue was purified by flash chromatography on $\mathrm{SiO}_{2}(4: 1 \rightarrow 1: 1$, petrol/EtOAc gradient elution) to give $\mathbf{2}(7 \mathrm{mg}, 50 \%)$ as a fine red-orange powder.

\section{Data for 2:}

$\mathbf{R}_{\mathbf{f}}=0.20(1: 1 \mathrm{petrol} / \mathrm{EtOAc})$

IR (neat): 3371, 2971, 1622, 1559, 1437, 1325, $1248 \mathrm{~cm}^{-1}$.

${ }^{1}$ H NMR (600 MHz, CDCl $)$ ): $\delta 12.24$ (s, 1H), 8.17 (s, 1H), 7.46 (s, 1H), 7.20 (s, 1H), 2.35 - 2.28 (m, 1H), $2.12(\mathrm{~s}, 3 \mathrm{H}), 1.98-1.92(\mathrm{~m}, 1 \mathrm{H}), 1.83-1.79(\mathrm{~m}, 1 \mathrm{H}), 1.64-1.58(\mathrm{~m}, 2 \mathrm{H}), 1.57(\mathrm{~s}, 3 \mathrm{H})$, $1.49(\mathrm{~s}, 3 \mathrm{H}), 1.16(\mathrm{~s}, 3 \mathrm{H})$.

${ }^{13}$ C NMR (150 MHz, $\left.\mathbf{C D C l}_{3}\right): \delta 183.4,182.5,162.3,161.6,154.5,135.5,131.6,127.1,117.0$, $116.3,108.5,108.0,82.3,68.4,42.5,35.8,30.7,26.7,20.2,19.5,7.8$.

HRMS (ESI): calculated for $\mathrm{C}_{21} \mathrm{H}_{21} \mathrm{O}_{6} 369.1338[\mathrm{M}-\mathrm{H}]^{-}$, found 369.1339 . 


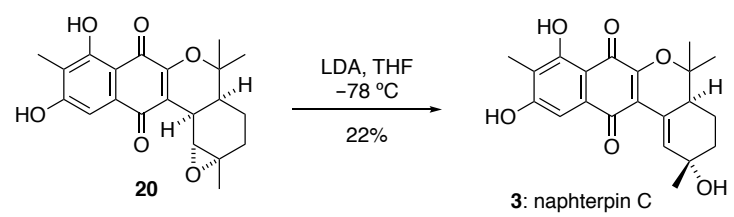

To a solution of diisopropylamine $(0.09 \mathrm{~mL}, 0.61 \mathrm{mmol})$ in THF $(3 \mathrm{~mL})$ at $0{ }^{\circ} \mathrm{C}$ was added $n$-BuLi (2.5 $\mathrm{M}$ in hexanes, $0.24 \mathrm{~mL}, 0.61 \mathrm{mmol}$ ). The reaction was stirred at $0{ }^{\circ} \mathrm{C}$ for $5 \mathrm{~min}$ prior to being cooled to $-78{ }^{\circ} \mathrm{C}$. A solution of $\mathbf{2 0}(45 \mathrm{mg}, 0.12 \mathrm{mmol})$ in THF (2 mL) was added dropwise to the reaction. The reaction was stirred at $-78^{\circ} \mathrm{C}$ for $2 \mathrm{~h}$. The mixture was quenched with saturated $\mathrm{NH}_{4} \mathrm{Cl}$ solution $(10 \mathrm{~mL})$ and extracted with EtOAc $(3 \times 10 \mathrm{~mL})$. The combined organic extracts were washed with saturated brine $(30 \mathrm{~mL})$, dried over anhydrous $\mathrm{MgSO}_{4}$, filtered and concentrated in vacuo. The residue was purified by flash chromatography on $\mathrm{SiO}_{2}(4: 1 \rightarrow 1: 1$, petrol/EtOAc gradient elution) to give 3 (10 $\mathrm{mg}, 22 \%)$ as a fine orange powder.

\section{Data for 3:}

$\mathbf{R}_{\mathbf{f}}=0.20$ (1:1 petrol/EtOAc)

IR (neat): 3451, 2925, 1633, 1597, 1436, 1325, 1288, 1247, $1076 \mathrm{~cm}^{-1}$.

${ }^{1}$ H-NMR (600 MHz, acetone-d 6 ): $\delta 12.43(\mathrm{~s}, 1 \mathrm{H}), 7.45$ (t, $\left.J=1.8 \mathrm{~Hz}, 1 \mathrm{H}\right), 7.15$ (s, 1H), 2.42 (ddd, $J=11.1,5.9,2.1 \mathrm{~Hz}, 1 \mathrm{H}), 2.11(\mathrm{~s}, 3 \mathrm{H}), 2.01-1.93(\mathrm{~m}, 1 \mathrm{H}), 1.89$ (dtd, $J=12.6,4.2,3.8,1.5$ $\mathrm{Hz}, 1 \mathrm{H}), 1.76(\mathrm{td}, J=13.4,12.9,3.4 \mathrm{~Hz}, 1 \mathrm{H}), 1.51(\mathrm{~s}, 3 \mathrm{H}), 1.43-1.36(\mathrm{~m}, 1 \mathrm{H}), 1.32(\mathrm{~s}, 3 \mathrm{H}), 1.18$ $(\mathrm{s}, 3 \mathrm{H})$.

${ }^{13}$ C-NMR (150 MHz, acetone-d $\left.{ }_{6}\right): \delta 183.8,183.4,163.4,162.7,162.4,154.4,140.9,133.0,124.6$, $117.3,116.9,116.8,108.1,108.0,82.2,43.4,37.9,26.6,23.7,19.6,7.9$.

${ }^{1}$ H-NMR (600 MHz, DMSO-d 6 ): $\delta 12.30$ (s, 1H), 7.31 (t, $J=1.7$ Hz, 1H), 7.07 (s, 1H), 4.80 (s, 1H), 2.35 (ddd, $J=11.1,6.0,2.0 \mathrm{~Hz}, 1 \mathrm{H}), 2.01$ (s, 3H), $1.88-1.83(\mathrm{~m}, 1 \mathrm{H}), 1.78-1.73(\mathrm{~m}, 1 \mathrm{H})$, $1.66-1.60(\mathrm{~m}, 1 \mathrm{H}), 1.45(\mathrm{~s}, 3 \mathrm{H}), 1.31-1.26(\mathrm{~m}, 1 \mathrm{H}), 1.22(\mathrm{~s}, 3 \mathrm{H}), 1.09$ (s, 3H).

${ }^{13}$ C-NMR (150 MHz, DMSO-d $)$ ): $\delta$ 182.6, 182.1, 162.9, 161.1, 153.1, 140.0, 131.4, 122.9, 115.9, 115.4, 107.4, 107.0, 81.5, 68.0, 41.9, 36.7, 28.9, 26.0, 22.1, 19.1, 7.8.

HRMS (ESI): calculated for $\mathrm{C}_{21} \mathrm{H}_{21} \mathrm{O}_{6} 369.1344$ [M-H] $]^{-}$, found 369.1338 . 


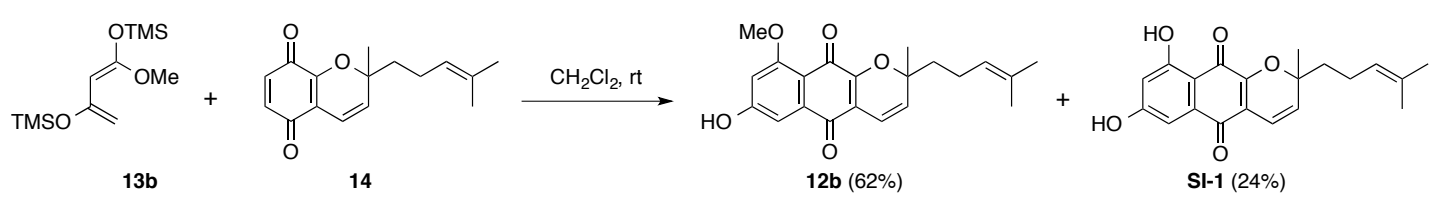

To a solution of $14(132 \mathrm{mg}, 0.51 \mathrm{mmol})$ in $\mathrm{CH}_{2} \mathrm{Cl}_{2}(5 \mathrm{~mL})$ at room temperature was added $\mathbf{1 3 \mathbf { b } ^ { 4 }}$ (182 $\mathrm{mg}, 0.66 \mathrm{mmol})$. The reaction mixture was stirred at room temperature for $2 \mathrm{~h}$. The mixture was quenched with $1 \mathrm{M} \mathrm{HCl}(10 \mathrm{~mL})$ and stirred at room temperature for $1 \mathrm{~h}$. The mixture was extracted with EtOAc $(3 \times 5 \mathrm{~mL})$. The combined organic extracts were washed with saturated brine $(20 \mathrm{~mL})$, dried over anhydrous $\mathrm{MgSO}_{4}$, filtered and concentrated in vacuo. The residue was purified by flash chromatography on $\mathrm{SiO}_{2}(20: 1 \rightarrow 9: 1$, petrol/EtOAc gradient elution) to give $\mathbf{1 2 b}(112 \mathrm{mg}, 62 \%)$ as a red solid and SI-1 (41 mg, 24\%) as a red oil.

\section{Data for 12b:}

$\mathbf{R}_{\mathbf{f}}=0.10(3: 2 \mathrm{petrol} / \mathrm{EtOAc})$

IR (neat): $\mathrm{cm}^{-1} 3237,2932,1640,1584,1438,1415,1347,1216 \mathrm{~cm}^{-1}$.

${ }^{1}$ H NMR (500 MHz, CDCl $): \delta 7.41(\mathrm{~d}, J=2.4 \mathrm{~Hz}, 1 \mathrm{H}), 6.76(\mathrm{~d}, J=2.4 \mathrm{~Hz}, 1 \mathrm{H}), 6.59(\mathrm{~d}, J=10.1$ $\mathrm{Hz}, 1 \mathrm{H}), 5.57$ (d, $J=10.1 \mathrm{~Hz}, 1 \mathrm{H}), 5.04(\mathrm{t}, J=6.3 \mathrm{~Hz}, 1 \mathrm{H}), 3.93(\mathrm{~s}, 3 \mathrm{H}), 2.16-2.01(\mathrm{~m}, 2 \mathrm{H}), 1.95$ $-1.85(\mathrm{~m}, 1 \mathrm{H}), 1.70-1.62(\mathrm{~m}, 1 \mathrm{H}), 1.62(\mathrm{~s}, 3 \mathrm{H}), 1.53(\mathrm{~s}, 3 \mathrm{H}), 1.48(\mathrm{~s}, 3 \mathrm{H})$.

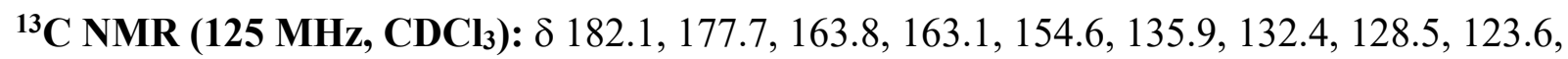
$115.8,115.4,112.6,108.0,103.9,83.7,56.5,41.7,27.6,25.7,22.8,17.8$.

HRMS (ESI): calculated for $\mathrm{C}_{21} \mathrm{H}_{23} \mathrm{O}_{5} 355.1545[\mathrm{M}+\mathrm{H}]^{+}$, found 355.1541 .

\section{Data for SI-1:}

$\mathbf{R}_{\mathbf{f}}=0.50$ (3:2 petrol/EtOAc)

IR (neat): $\mathrm{cm}^{-1} 3287,2969,2925,2855,1614,1575,1452,1400,1317,1225 \mathrm{~cm}^{-1}$.

${ }^{1}$ H NMR (500 MHz, CDCl $): \delta 12.01(\mathrm{~s}, 1 \mathrm{H}), 7.26(\mathrm{~d}, J=2.2 \mathrm{~Hz}, 1 \mathrm{H}), 6.62(\mathrm{~d}, J=10.1 \mathrm{~Hz}, 1 \mathrm{H})$, $6.60(\mathrm{~d}, J=2.2 \mathrm{~Hz}, 1 \mathrm{H}), 5.63(\mathrm{~d}, J=10.1 \mathrm{~Hz}, 1 \mathrm{H}), 5.07$ (t, $J=7.0 \mathrm{~Hz}, 1 \mathrm{H}), 2.10(\mathrm{q}, J=7.6 \mathrm{~Hz}$, 2H), $1.97-1.90(\mathrm{~m}, 1 \mathrm{H}), 1.70-1.65(\mathrm{~m}, 1 \mathrm{H}), 1.63(\mathrm{~s}, 3 \mathrm{H}), 1.55(\mathrm{~s}, 3 \mathrm{H}), 1.51(\mathrm{~s}, 3 \mathrm{H})$.

${ }^{13}$ C NMR (125 MHz, $\left.\mathbf{C D C l}_{3}\right): \delta 182.6,181.5,164.8,164.1,153.8,133.7,132.6,129.5,123.5$, $117.5,115.8,109.4,109.2,108.0,84.0,41.8,27.8,25.7,22.8,17.8$.

HRMS (ESI): calculated for $\mathrm{C}_{20} \mathrm{H}_{21} \mathrm{O}_{5} 341.1389[\mathrm{M}+\mathrm{H}]^{+}$, found 341.1387 .

\footnotetext{
${ }^{4}$ Prepared according to: Barker, D.; Brimble, M. A.; Do, P.; Turner, P. Tetrahedron 2003, 59, 2441.
} 


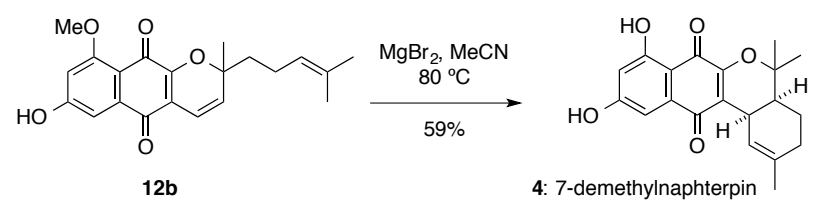

To a solution of $\mathbf{1 2 b}$ (35 mg, $0.10 \mathrm{mmol})$ in $\mathrm{MeCN}(4 \mathrm{~mL})$ at room temperature was added $\mathrm{MgBr}_{2}$ (91 mg, $0.50 \mathrm{mmol}$ ). The reaction was stirred at $80^{\circ} \mathrm{C}$ for $14 \mathrm{~h}$. The mixture was cooled to room temperature, quenched with $1 \mathrm{M} \mathrm{HCl}(10 \mathrm{~mL})$ and extracted with EtOAc $(3 \times 10 \mathrm{~mL})$. The combined organic extracts were washed with saturated brine $(30 \mathrm{~mL})$, dried over anhydrous $\mathrm{MgSO}_{4}$, filtered and concentrated in vacuo. The residue was purified by flash chromatography on $\mathrm{SiO}_{2}(9: 1 \rightarrow 4: 1$, petrol/EtOAc gradient elution) to give 4 (20 mg, 59\%) as an orange solid.

\section{Data for 4:}

$\mathbf{R}_{\mathbf{f}}=0.60(1: 1, \mathrm{petrol} / \mathrm{EtOAc})$

$\mathbf{M p}=255-257^{\circ} \mathrm{C}$

IR (neat): 3344, 2921, 1625, 1600, 1572, 1458, 1335, 1285, 1220, 1159, $1126 \mathrm{~cm}^{-1}$.

${ }^{1}$ H NMR (500 MHz, CDCl$): \delta 11.91(s, 1 H), 7.19(\mathrm{~d}, J=2.3 \mathrm{~Hz}, 1 \mathrm{H}), 6.89$ (s, 1H), 6.56 (d, $J=$ $2.4 \mathrm{~Hz}, 1 \mathrm{H}), 6.03$ (d, $J=3.8 \mathrm{~Hz}, 1 \mathrm{H}), 3.49$ (t, $J=5.9 \mathrm{~Hz}, 1 \mathrm{H}), 1.99(\mathrm{dd}, J=20.7,6.8 \mathrm{~Hz}, 2 \mathrm{H}), 1.93$ (dd, $J=5.6,2.2 \mathrm{~Hz}, 1 \mathrm{H}), 1.77(\mathrm{ddd}, J=12.1,6.2,2.8 \mathrm{~Hz}, 1 \mathrm{H}), 1.67$ (s, 3H), 1.55 (s, 3H), 1.34 (s, $3 \mathrm{H}), 1.33-1.28(\mathrm{~m}, 1 \mathrm{H})$.

${ }^{13}$ C NMR (125 MHz, $\left.\mathbf{C D C l}_{3}\right): \delta$ 184.1, 183.2, 164.6, 153.4, 136.3, 135.0, 123.9, 120.1, 108.9, $108.8,107.4,80.9,39.9,31.3,29.9,29.8,25.8,25.2,23.7,20.5$.

HRMS (ESI): calculated for $\mathrm{C}_{20} \mathrm{H}_{21} \mathrm{O}_{5} 341.1389[\mathrm{M}+\mathrm{H}]^{+}$, found 341.1388. 


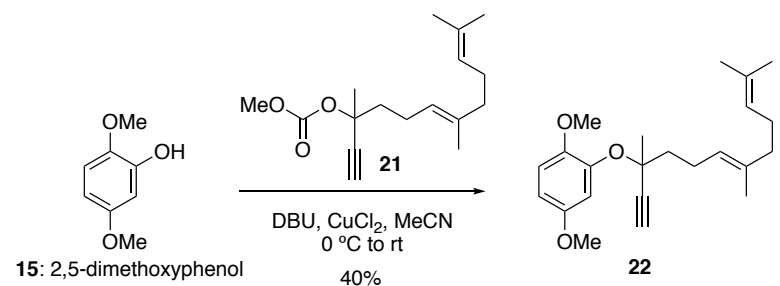

To a solution of $15(2.07 \mathrm{~g}, 13.4 \mathrm{mmol})$ in $\mathrm{MeCN}(200 \mathrm{~mL})$ at $0{ }^{\circ} \mathrm{C}$ was added DBU $(2.17 \mathrm{~mL}, 17.5$ mmol) dropwise $\mathrm{CuCl}_{2}(18 \mathrm{mg}, 0.013 \mathrm{mmol})$ and $21^{5}(4.48 \mathrm{~g}, 16.1 \mathrm{mmol})$. The reaction mixture was stirred at $0{ }^{\circ} \mathrm{C}$ for $8 \mathrm{~h}$ prior to being warmed to $\mathrm{rt}$ and stirred for a further $16 \mathrm{~h}$. The mixture was quenched with saturated $\mathrm{NH}_{4} \mathrm{Cl}$ solution $(200 \mathrm{~mL})$ and extracted with EtOAc $(3 \times 150 \mathrm{~mL})$. The combined organic extracts were washed with saturated brine $(300 \mathrm{~mL})$, dried over anhydrous $\mathrm{MgSO}_{4}$, filtered and concentrated in vacuo. The residue was purified by flash chromatography on $\mathrm{SiO}_{2}(20: 1$, petrol/EtOAc gradient elution) to give $22(1.89 \mathrm{~g}, 40 \%)$ as a pale yellow oil.

\section{Data for 22:}

$\mathbf{R}_{\mathbf{f}}=0.55$ (4:1, petrol/EtOAc)

IR (neat): 3286, 2932, 1504, 1443, 1260, $1223 \mathrm{~cm}^{-1}$.

${ }^{1}$ H NMR (600 MHz, CDCl 3$): \delta 7.10(\mathrm{~d}, J=3.0 \mathrm{~Hz}, 1 \mathrm{H}), 6.81(\mathrm{~d}, J=8.8 \mathrm{~Hz}, 1 \mathrm{H}), 6.56(\mathrm{dd}, J=$ 8.8f, $3.0 \mathrm{~Hz}, 1 \mathrm{H}), 5.17$ (tq, $J=7.1,1.3 \mathrm{~Hz}, 1 \mathrm{H}), 5.10$ (t, $J=6.9 \mathrm{~Hz}, 1 \mathrm{H}), 3.77$ (s, 3H), 3.76 (s, 3H), $2.58(\mathrm{~s}, 1 \mathrm{H}), 2.39-2.23(\mathrm{~m}, 2 \mathrm{H}), 2.10-2.04(\mathrm{~m}, 2 \mathrm{H}), 2.01-1.96(\mathrm{~m}, 3 \mathrm{H}), 1.88(\mathrm{ddd}, J=13.5$, 11.8, 5.1 Hz, 1H), $1.68(\mathrm{~s}, 3 \mathrm{H}), 1.63(\mathrm{~s}, 3 \mathrm{H}), 1.60(\mathrm{~s}, 3 \mathrm{H}), 1.58(\mathrm{~s}, 3 \mathrm{H})$.

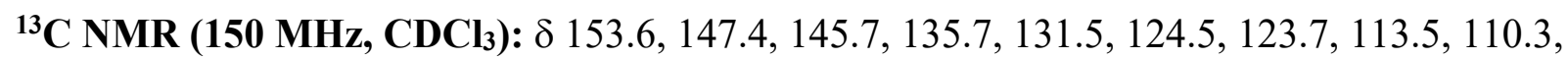
$107.8,85.5,77.1,75.0,56.9,55.8,42.5,39.8,26.8,26.7,25.8,23.3,17.8,16.1$.

HRMS (ESI): calculated for $\mathrm{C}_{23} \mathrm{H}_{33} \mathrm{O}_{3} 357.2430[\mathrm{M}+\mathrm{H}]^{+}$, found 357.2416.

5 Prepared from trans,trans-geranylacetone according to: (a) Tsuji, J.; Sugiura, T.; Minami, I. Synthesis 1987, 603. (b) Gassner, C.; Hesse, R.; Schmidt, A. W.; Knolker, H.-J. Org. Biomol. Chem. 2014, 12, 6490 . 


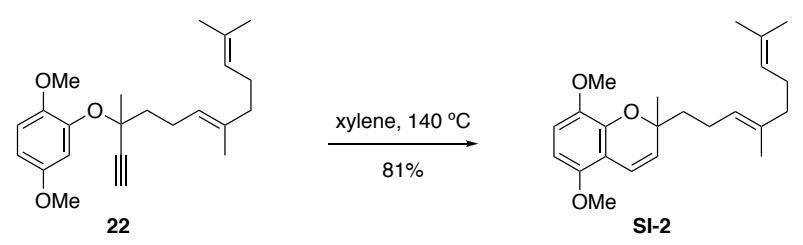

A solution of $22(200 \mathrm{mg}, 0.56 \mathrm{mmol})$ in xylene $(15 \mathrm{~mL})$ was stirred at $140{ }^{\circ} \mathrm{C}$ for $2 \mathrm{~h}$. The mixture was cooled to room temperature and concentrated in vacuo. The residue was purified by flash chromatography on $\mathrm{SiO}_{2}(20: 1$, petrol/EtOAc) to give SI-2 (162 g, 81\%) as a yellow oil.

\section{Data for SI-2:}

$\mathbf{R}_{\mathbf{f}}=0.50$ (4:1, petrol/EtOAc)

IR (neat): 2967, 2925, 2836, 1491, 1463, 1439, $1385 \mathrm{~cm}^{-1}$.

${ }^{1}$ H NMR (600 MHz, CDCl $): \delta 6.69(\mathrm{dd}, J=12.4,9.5 \mathrm{~Hz}, 2 \mathrm{H}), 6.30(\mathrm{~d}, J=8.9 \mathrm{~Hz}, 1 \mathrm{H}), 5.56(\mathrm{~d}, J$ $=10.1 \mathrm{~Hz}, 1 \mathrm{H}), 5.10(\mathrm{tq}, J=7.1,1.3 \mathrm{~Hz}, 1 \mathrm{H}), 5.07(\mathrm{t}, J=7.0 \mathrm{~Hz}, 1 \mathrm{H}), 3.81(\mathrm{~s}, 3 \mathrm{H}), 3.78(\mathrm{~s}, 3 \mathrm{H})$, $2.16-2.11(\mathrm{~m}, 2 \mathrm{H}), 2.06-2.01(\mathrm{~m}, 2 \mathrm{H}), 1.96-1.92(\mathrm{~m}, 2 \mathrm{H}), 1.86-1.80(\mathrm{~m}, 1 \mathrm{H}), 1.71-1.63$ (overlapping m, 1H), $1.67(\mathrm{~s}, 3 \mathrm{H}), 1.58(\mathrm{~s}, 3 \mathrm{H}), 1.56(\mathrm{~s}, 3 \mathrm{H}), 1.44(\mathrm{~s}, 3 \mathrm{H})$.

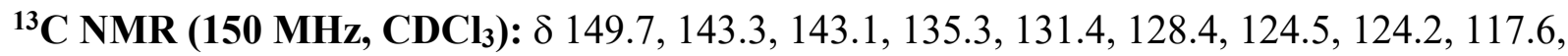
$113.0,111.8,101.6,78.5,57.2,56.0,40.8,39.8,26.8,26.3,25.8,22.8,17.8,16.0$.

HRMS (ESI): calculated for $\mathrm{C}_{23} \mathrm{H}_{33} \mathrm{O}_{3} 357.2430[\mathrm{M}+\mathrm{H}]^{+}$, found 357.2408. 


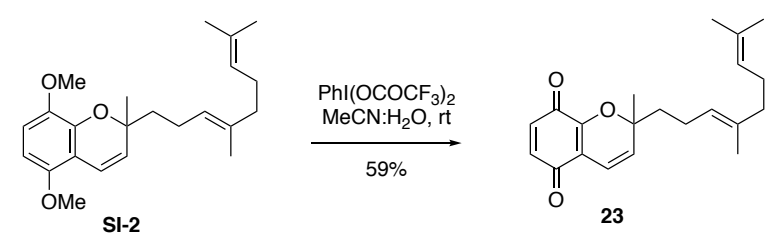

To a solution of SI-2 (1.32 g, $3.70 \mathrm{mmol})$ in $\mathrm{MeCN}: \mathrm{H}_{2} \mathrm{O}(90 \mathrm{~mL}, 2: 1 \mathrm{v} / \mathrm{v})$ at room temperature was added $\mathrm{PhI}\left(\mathrm{OCOCF}_{3}\right)_{2}(3.50 \mathrm{~g}, 8.15 \mathrm{mmol})$. The reaction mixture was stirred at room temperature for 5 min. The mixture was quenched with saturated $\mathrm{NaHCO}_{3}$ solution $(100 \mathrm{~mL})$ and extracted with $\mathrm{Et}_{2} \mathrm{O}$ $(3 \times 80 \mathrm{~mL})$. The combined organic extracts were washed with $\mathrm{H}_{2} \mathrm{O}(200 \mathrm{~mL})$ and saturated brine (200 mL), dried over anhydrous $\mathrm{MgSO}_{4}$, filtered and concentrated in vacuo. The residue was purified by flash chromatography on $\mathrm{SiO}_{2}(20: 1 \rightarrow 9: 1$, petrol/EtOAc gradient elution) to give 23 (707 mg, $59 \%)$ as a dark red oil.

\section{Data for 23:}

$\mathbf{R}_{\mathbf{f}}=0.45$ (4:1, petrol/EtOAc)

IR (neat): 2970, 2917, 1674, 1647, 1575, 1447, 1413, $1335 \mathrm{~cm}^{-1}$.

${ }^{1}$ H NMR (600 MHz, CDCl $): \delta 6.64(\mathrm{~s}, 2 \mathrm{H}), 6.47(\mathrm{~d}, J=10.1 \mathrm{~Hz}, 1 \mathrm{H}), 5.59$ (d, $\left.J=10.1 \mathrm{~Hz}, 1 \mathrm{H}\right)$, $5.12-5.04(\mathrm{~m}, 2 \mathrm{H}), 2.14-2.06(\mathrm{~m}, 2 \mathrm{H}), 2.07-2.00(\mathrm{~m}, 2 \mathrm{H}), 1.97-1.88(\mathrm{~m}, 3 \mathrm{H}), 1.71-1.63$ (overlapping m, 1H), 1.67 (s, 3H), 1.59 (s, 3H), 1.56 (s, 3H), 1.48 (s, 3H).

${ }^{13}$ C NMR (150 MHz, $\left.\mathbf{C D C l}_{3}\right): \delta 184.4,181.6,150.9,136.1,136.0,134.7,131.5,129.3,124.3$, $123.3,115.8,115.4,83.3,41.6,39.7,27.6,26.7,25.8,22.6,17.8,16.1$.

HRMS (ESI): calculated for $\mathrm{C}_{21} \mathrm{H}_{27} \mathrm{O}_{3} 327.1960[\mathrm{M}+\mathrm{H}]^{+}$, found 327.1948 . 


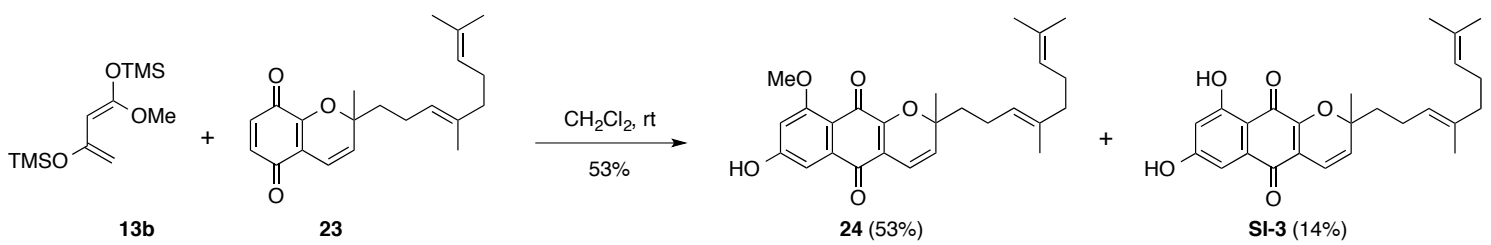

To a solution of $23(597 \mathrm{mg}, 1.83 \mathrm{mmol})$ in $\mathrm{CH}_{2} \mathrm{Cl}_{2}(12 \mathrm{~mL})$ at room temperature was added 13b $(619 \mathrm{mg}, 2.38 \mathrm{mmol})$. The reaction mixture was stirred at room temperature for $20 \mathrm{~min}$. The mixture was quenched with $1 \mathrm{M} \mathrm{HCl}(10 \mathrm{~mL})$ and stirred at room temperature for $1 \mathrm{~h}$. The mixture was extracted with EtOAc $(3 \times 30 \mathrm{~mL})$. The combined organic extracts were washed with saturated brine $(100 \mathrm{~mL})$, dried over anhydrous $\mathrm{MgSO}_{4}$, filtered and concentrated in vacuo. The residue was purified by flash chromatography on $\mathrm{SiO}_{2}(9: 1 \rightarrow 1: 1$, petrol/EtOAc gradient elution) to give $\mathbf{2 4}$ (408 mg, 53 $\%$ ) as an amorphous red solid and SI-3 (106 mg, $14 \%$ ) as a red oil.

\section{Data for 24:}

$\mathbf{R}_{\mathbf{f}}=0.10$ (4:1, petrol/EtOAc)

IR (neat): 3252, 2970, 2925, 1628, 1597, 1568, 1439, 1349, $1222 \mathrm{~cm}^{-1}$.

${ }^{1}$ H NMR (600 MHz, CDCl $): \delta 7.45$ (br s, $\left.1 \mathrm{H}\right), 7.38(\mathrm{~d}, J=2.3 \mathrm{~Hz}, 1 \mathrm{H}), 6.72(\mathrm{~d}, J=2.4 \mathrm{~Hz}, 1 \mathrm{H})$, $6.60(\mathrm{~d}, J=10.0 \mathrm{~Hz}, 1 \mathrm{H}), 5.58(\mathrm{~d}, J=10.0 \mathrm{~Hz}, 1 \mathrm{H}), 5.11-5.03(\mathrm{~m}, 2 \mathrm{H}), 3.95(\mathrm{~s}, 3 \mathrm{H}), 2.17-2.06$ (m, 2H), $2.05-1.99(\mathrm{~m}, 2 \mathrm{H}), 1.96-1.89(\mathrm{~m}, 3 \mathrm{H}), 1.71-1.67(\mathrm{~m}, 1 \mathrm{H}), 1.66(\mathrm{~s}, 3 \mathrm{H}), 1.58(\mathrm{~s}, 3 \mathrm{H})$, $1.55(\mathrm{~s}, 3 \mathrm{H}), 1.48(\mathrm{~s}, 3 \mathrm{H})$.

${ }^{13}$ C NMR (150 MHz, $\left.\mathbf{C D C l}_{3}\right): \delta 182.1,177.7,164.0,163.1,154.6,136.0,135.8,131.5,128.6$, $124.4,123.3,115.7,115.4,112.4,108.1,103.9,83.7,56.4,41.7,39.7,27.6,26.7,25.8,22.6,17.8$, 16.1 .

HRMS (ESI): calculated for $\mathrm{C}_{26} \mathrm{H}_{29} \mathrm{O}_{5} 421.2015$ [M-H] $]^{-}$, found 421.2014 .

\section{Data for SI-3:}

$\mathbf{R}_{\mathbf{f}}=0.30$ (4:1, petrol/EtOAc)

IR (neat): 3312, 2969, 2924, 1617, 1577, 1453, 1399, 1318, $1226 \mathrm{~cm}^{-1}$.

${ }^{1}$ H NMR (600 MHz, $\left.\mathbf{C D C l}_{3}\right): \delta 12.04(\mathrm{~s}, 1 \mathrm{H}), 7.23(\mathrm{~d}, J=2.4 \mathrm{~Hz}, 1 \mathrm{H}), 6.83(\mathrm{~s}, 1 \mathrm{H}), 6.63(\mathrm{~d}, J=$ $10.1 \mathrm{~Hz}, 1 \mathrm{H}), 6.59(\mathrm{~d}, J=2.5 \mathrm{~Hz}, 1 \mathrm{H}), 5.65(\mathrm{~d}, J=10.1 \mathrm{~Hz}, 1 \mathrm{H}), 5.09$ (t, $J=7.0 \mathrm{~Hz}, 1 \mathrm{H}), 5.06(\mathrm{t}, J$ $=6.9 \mathrm{~Hz}, 1 \mathrm{H}), 2.17-2.09(\mathrm{~m}, 2 \mathrm{H}), 2.06-1.99(\mathrm{~m}, 2 \mathrm{H}), 1.98-1.91(\mathrm{~m}, 3 \mathrm{H}), 1.74-1.68(\mathrm{~m}, 1 \mathrm{H})$, $1.67(\mathrm{~s}, 3 \mathrm{H}), 1.58(\mathrm{~s}, 3 \mathrm{H}), 1.56(\mathrm{~s}, 3 \mathrm{H}), 1.52(\mathrm{~s}, 3 \mathrm{H})$.

${ }^{13}$ C NMR (150 MHz, $\left.\mathbf{C D C l}_{3}\right): \delta 182.7,181.3,164.7,163.5,153.6,136.2,133.8,131.6,129.6$, 124.3, 123.2, 117.6, 115.8, 109.4, 109.0, 107.9, 83.9, 41.7, 39.7, 27.7, 26.7, 25.8, 22.7, 17.8, 16.2.

HRMS (ESI): calculated for $\mathrm{C}_{25} \mathrm{H}_{29} \mathrm{O}_{5} 409.2015[\mathrm{M}+\mathrm{H}]^{+}$, found 409.2007. 

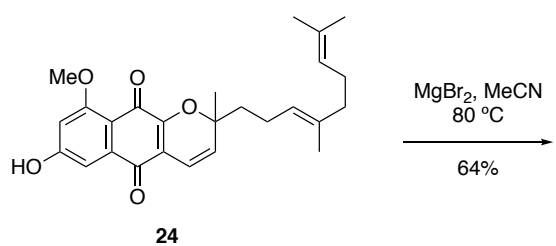

24

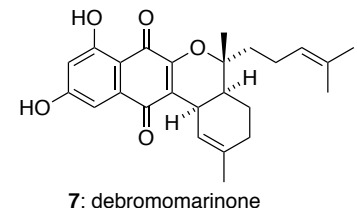

To a solution of $24(212 \mathrm{mg}, 0.50 \mathrm{mmol})$ in $\mathrm{MeCN}(20 \mathrm{~mL})$ at room temperature was added $\mathrm{MgBr}_{2}$ (462 mg, $2.51 \mathrm{mmol}$ ). The reaction was stirred at $80{ }^{\circ} \mathrm{C}$ for $14 \mathrm{~h}$. The mixture was cooled to room temperature, quenched with $1 \mathrm{M} \mathrm{HCl}(50 \mathrm{~mL})$ and extracted with EtOAc $(3 \times 30 \mathrm{~mL})$. The combined organic extracts were washed with saturated brine $(90 \mathrm{~mL})$, dried over anhydrous $\mathrm{MgSO}_{4}$, filtered and concentrated in vacuo. The residue was purified by flash chromatography on $\mathrm{SiO}_{2}(8: 1$, petrol/EtOAc) to give 7 (130 mg, $64 \%$ ) as an amorphous orange solid.

\section{Data for 7:}

$\mathbf{R}_{\mathbf{f}}=0.30$ (3:1, petrol/EtOAc)

IR (neat): 3300, 2969, 2929, 1630, 1583, 1324, 1221, 1160, 1007, $769 \mathrm{~cm}^{-1}$.

${ }^{1}$ H NMR (500 MHz, CDCl 3$): \delta 11.90(\mathrm{~s}, 1 \mathrm{H}), 7.22(\mathrm{~d}, J=2.3 \mathrm{~Hz}, 1 \mathrm{H}), 6.57(\mathrm{~d}, J=2.3 \mathrm{~Hz}, 1 \mathrm{H})$, $6.03(\mathrm{~d}, J=4.3 \mathrm{~Hz}, 1 \mathrm{H}), 5.02(\mathrm{t}, J=7.0 \mathrm{~Hz}, 1 \mathrm{H}), 3.45(\mathrm{~d}, J=6.0 \mathrm{~Hz}, 1 \mathrm{H}), 2.10-1.85(\mathrm{~m}, 6 \mathrm{H}), 1.71$ - $1.64(\mathrm{~m}, 2 \mathrm{H}), 1.67(\mathrm{~s}, 3 \mathrm{H}), 1.62(\mathrm{~s}, 3 \mathrm{H}), 1.56(\mathrm{~s}, 3 \mathrm{H}), 1.52(\mathrm{~s}, 3 \mathrm{H}), 1.38-1.30(\mathrm{~m}, 1 \mathrm{H})$.

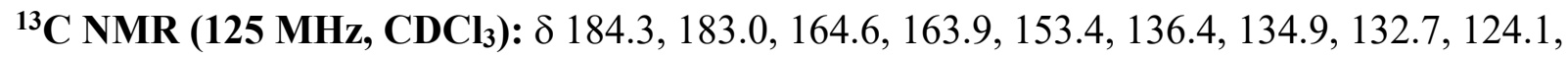
123.3, 120.1, 109.0, 108.7, 107.5, 83.3, 37.5, 36.9, 31.0, 29.9, 25.7, 23.7, 22.8, 22.4, 20.4, 17.8 .

HRMS (ESI): calculated for $\mathrm{C}_{25} \mathrm{H}_{29} \mathrm{O}_{5} 409.2015[\mathrm{M}+\mathrm{H}]^{+}$, found 409.2009. 

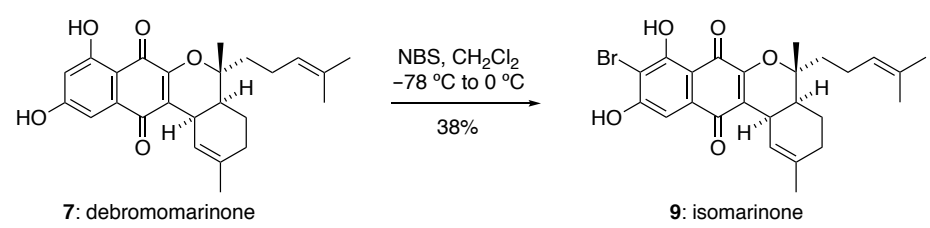

To a solution of $7(32 \mathrm{mg}, 0.08 \mathrm{mmol})$ in $\mathrm{CH}_{2} \mathrm{Cl}_{2}(2 \mathrm{~mL})$ at $-78{ }^{\circ} \mathrm{C}$ was added NBS $(21 \mathrm{mg}, 0.12$ mmol). The reaction mixture was stirred at $-78^{\circ} \mathrm{C}$ for $1 \mathrm{~h}$, prior to being warmed to $0{ }^{\circ} \mathrm{C}$ and stirred for a further $1.5 \mathrm{~h}$. The mixture was warmed to room temperature, quenched with saturated $\mathrm{Na}_{2} \mathrm{~S}_{2} \mathrm{O}_{3}$ solution $(10 \mathrm{~mL})$ and extracted with $\mathrm{CH}_{2} \mathrm{Cl}_{2}(3 \times 7 \mathrm{~mL})$. The combined organic extracts were washed with saturated brine $(20 \mathrm{~mL})$, dried over anhydrous $\mathrm{MgSO}_{4}$, filtered and concentrated in vacuo. The residue was purified by flash chromatography on $\mathrm{SiO}_{2}$ (8:1, petrol/EtOAc gradient elution) to give 9 (15 mg, $38 \%)$ as an amorphous orange solid.

\section{Data for 9:}

$\mathbf{R}_{\mathbf{f}}=0.25(3: 1$, petrol/EtOAc)

IR (neat): 2959, 2923, 1635, 1588, 1430, 1377, 1329, $1280 \mathrm{~cm}^{-1}$.

${ }^{1}$ H NMR (600 MHz, CDCl $\left.\mathbf{~ N}_{3}\right): \delta 12.72(\mathrm{~s}, 1 \mathrm{H}), 7.28(\mathrm{~s}, 1 \mathrm{H}), 6.51(\mathrm{~s}, 1 \mathrm{H}), 6.05(\mathrm{~d}, J=4.1 \mathrm{~Hz}, 1 \mathrm{H})$, $5.02(\mathrm{t}, J=7.0 \mathrm{~Hz}, 1 \mathrm{H}), 3.49-3.45(\mathrm{~m}, 1 \mathrm{H}), 2.08-1.97$ (m, 4H), $1.96-1.92$ (m, 1H), 1.89 (ddd, $J$ $=12.1,6.1,3.0 \mathrm{~Hz}, 1 \mathrm{H}), 1.68(\mathrm{~s}, 3 \mathrm{H}), 1.67-1.64$ (overlapping $\mathrm{m}, 2 \mathrm{H}), 1.63(\mathrm{~s}, 3 \mathrm{H}), 1.56(\mathrm{~s}, 3 \mathrm{H})$, $1.55(\mathrm{~s}, 3 \mathrm{H}), 1.35-1.28(\mathrm{~m}, 1 \mathrm{H})$.

${ }^{13}$ C NMR (150 MHz, $\left.\mathbf{C D C l}_{3}\right): \delta 183.3,182.9,160.0,159.5,152.7,136.5,133.3,132.7,124.9$, 123.2, 120.0, 108.9, 107.8, 103.3, 83.3, 37.6, 36.8, 31.1, 29.9, 25.7, 23.7, 22.8, 22.4, 20.4, 17.8 .

${ }^{1}$ H NMR (600 MHz, DMSO-d6): $\delta 12.54$ (s, 1H), 12.09 (br s, 1H), 7.08 (s, 1H), 5.95 (d, J =5.0 Hz, $1 \mathrm{H}), 5.03(\mathrm{t}, J=6.5 \mathrm{~Hz}, 1 \mathrm{H}), 3.36-3.34(\mathrm{~m}, 1 \mathrm{H}), 2.04-1.84(\mathrm{~m}, 6 \mathrm{H}), 1.61(\mathrm{~s}, 3 \mathrm{H}), 1.59-1.54$ (overlapping m, 2H), $1.55(\mathrm{~s}, 3 \mathrm{H}), 1.49(\mathrm{~s}, 3 \mathrm{H}), 1.42(\mathrm{~s}, 3 \mathrm{H}), 1.22-1.14(\mathrm{~m}, 1 \mathrm{H})$.

${ }^{13}$ C NMR (150 MHz, DMSO-d 6 ): $\delta$ 182.5, 182.1, 161.9, 159.7, 152.3, 135.4, 132.1, 131.1, 123.8, 123.1, 120.1, 107.4, 107.1, 101.8, 82.4, 36.6, 36.0, 30.4, 29.2, 25.3, 23.4, 22.0, 21.6, 19.7, 17.3.

HRMS (ESI): calculated for $\mathrm{C}_{25} \mathrm{H}_{28} \mathrm{BrO}_{5} 487.1120[\mathrm{M}+\mathrm{H}]^{+}$, found 487.1098 . 


\section{NMR spectra}

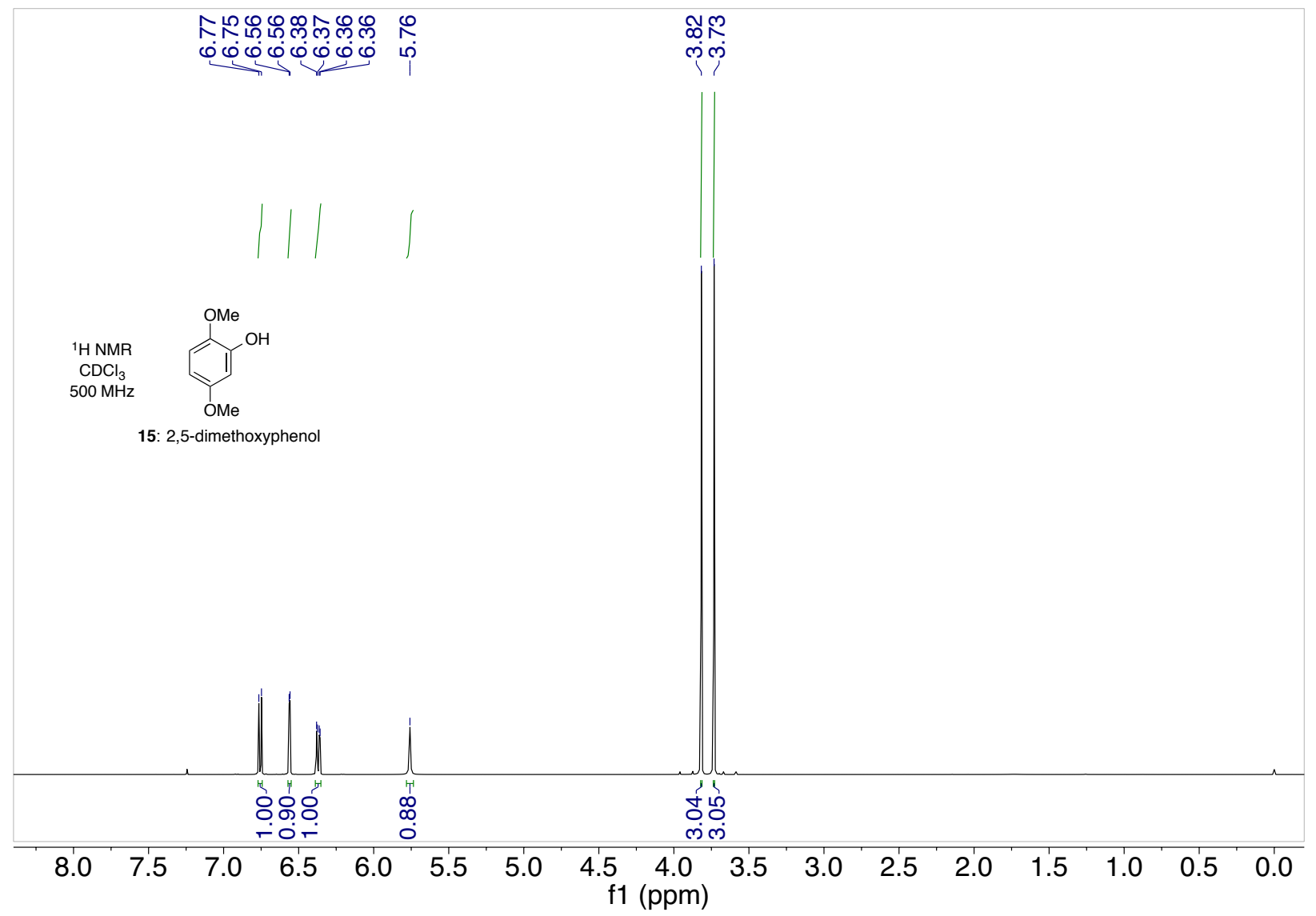

닫 훙유

${ }^{13} \mathrm{CNMR}$

$\mathrm{CDCl}_{3}$

$\mathrm{OMe}$

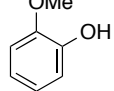

OMe

15: 2,5-dimethoxyphenol

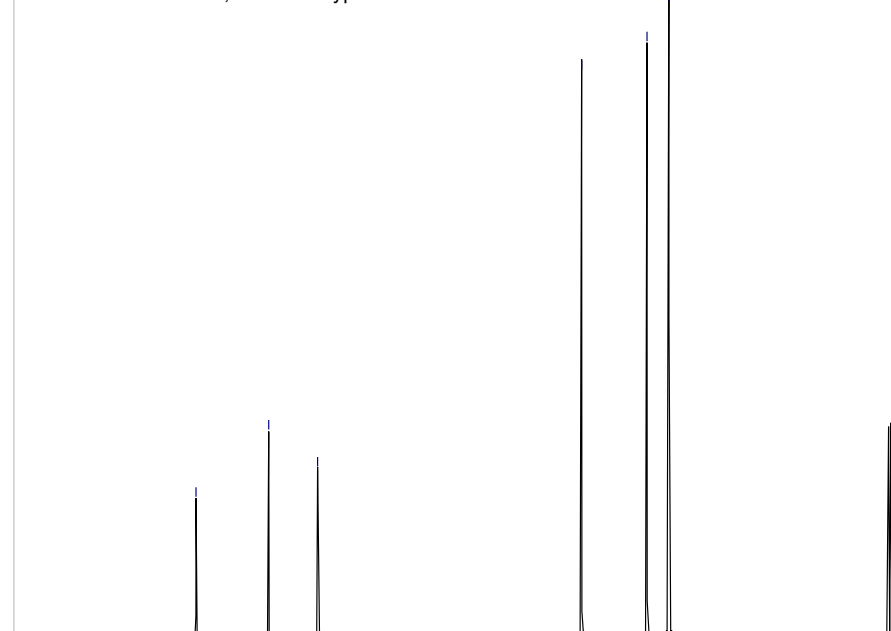

ติ่

น

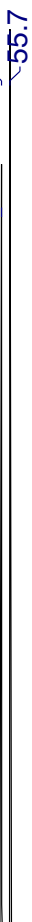

$\begin{array}{llllllllllllllllll}170 & 160 & 150 & 140 & 130 & 120 & 110 & 100 & \begin{array}{c}90 \\ \mathrm{f} 1(\mathrm{ppm})\end{array} & 70 & 60 & 50 & 40 & 30 & 20 & 10 & 0\end{array}$ 


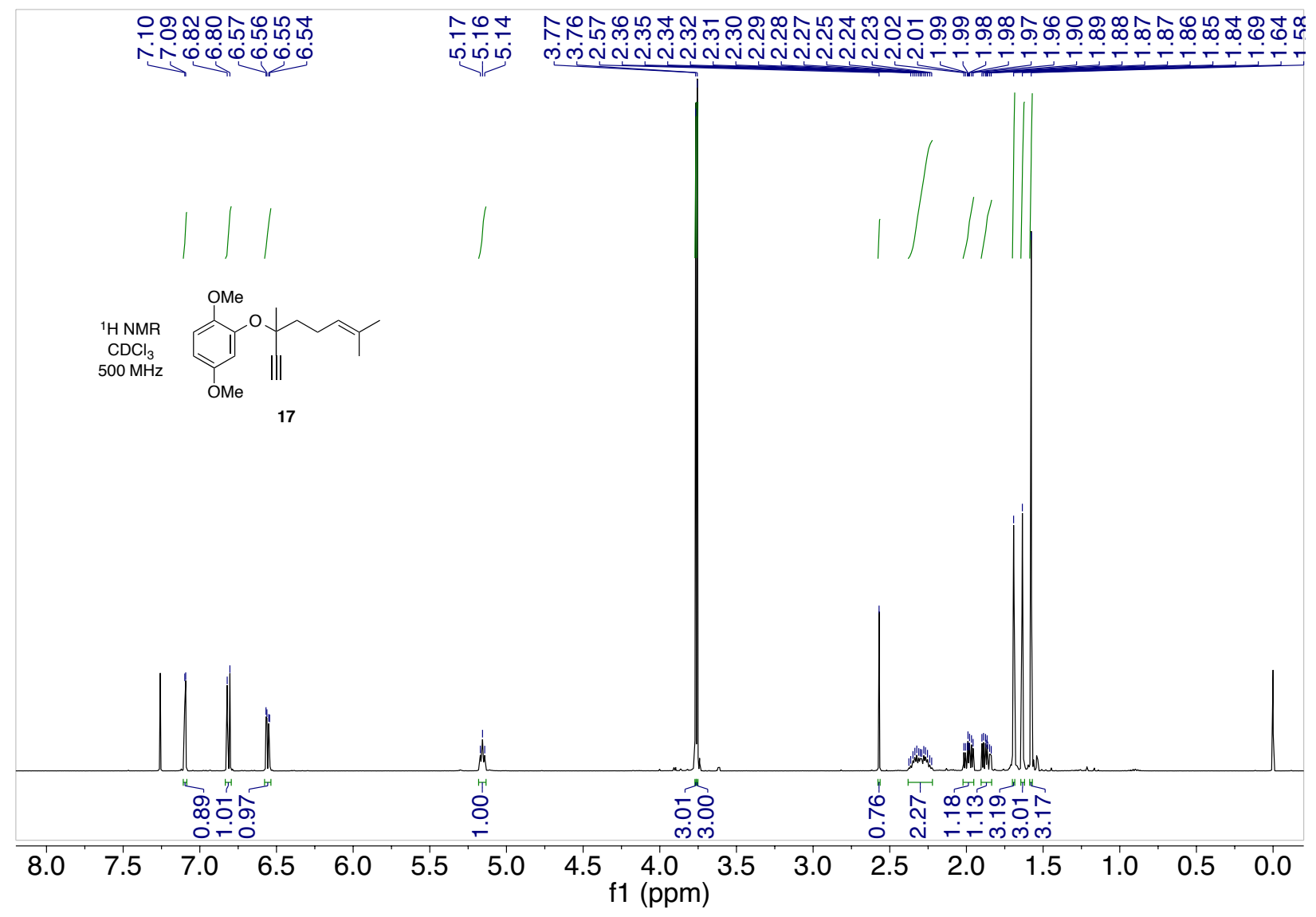

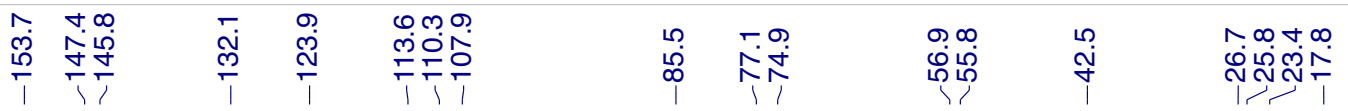
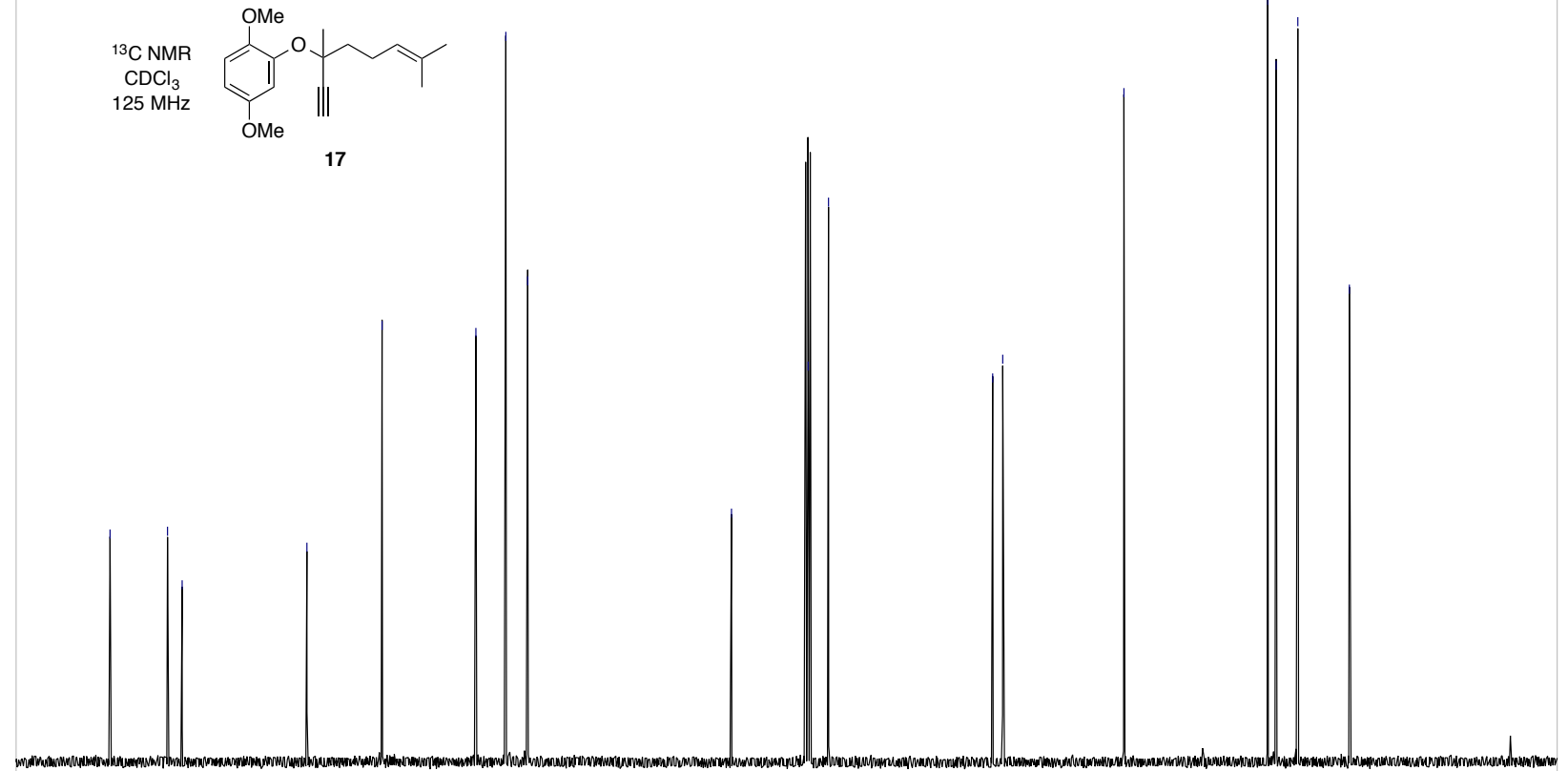

$\begin{array}{lllllllllllllllll}160 & 150 & 140 & 130 & 120 & 110 & 100 & 90 & \begin{array}{c}80 \\ \mathrm{f} 1\end{array}(\mathrm{ppm}) & 70 & 60 & 50 & 40 & 30 & 20 & 10 & 0\end{array}$ 


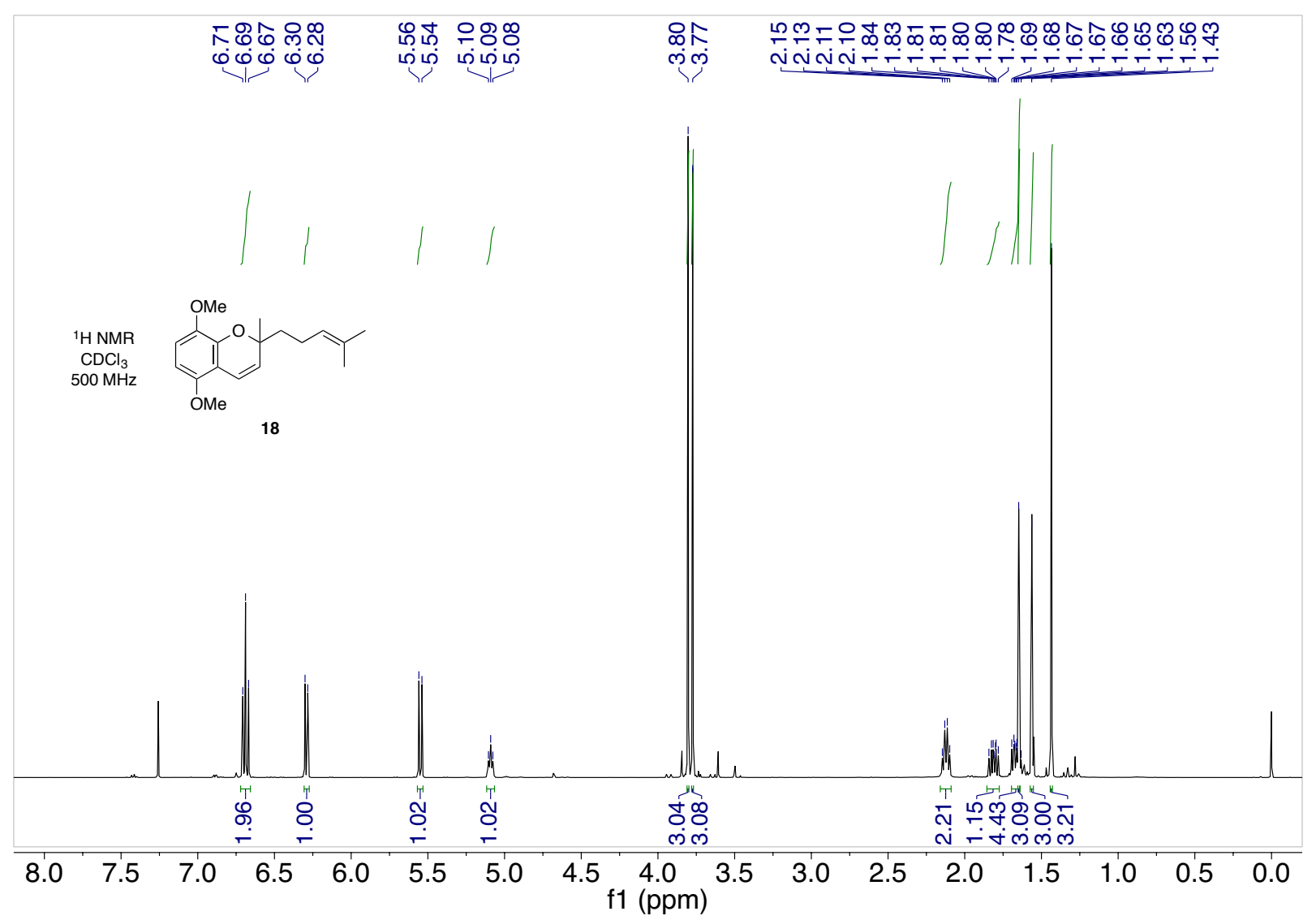

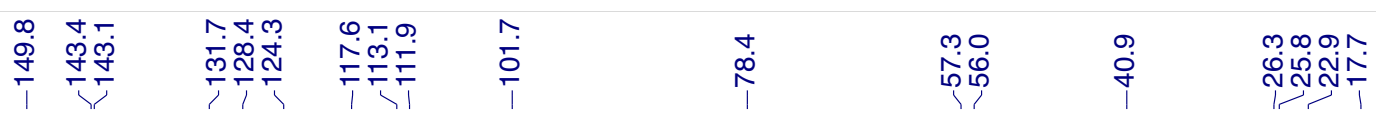
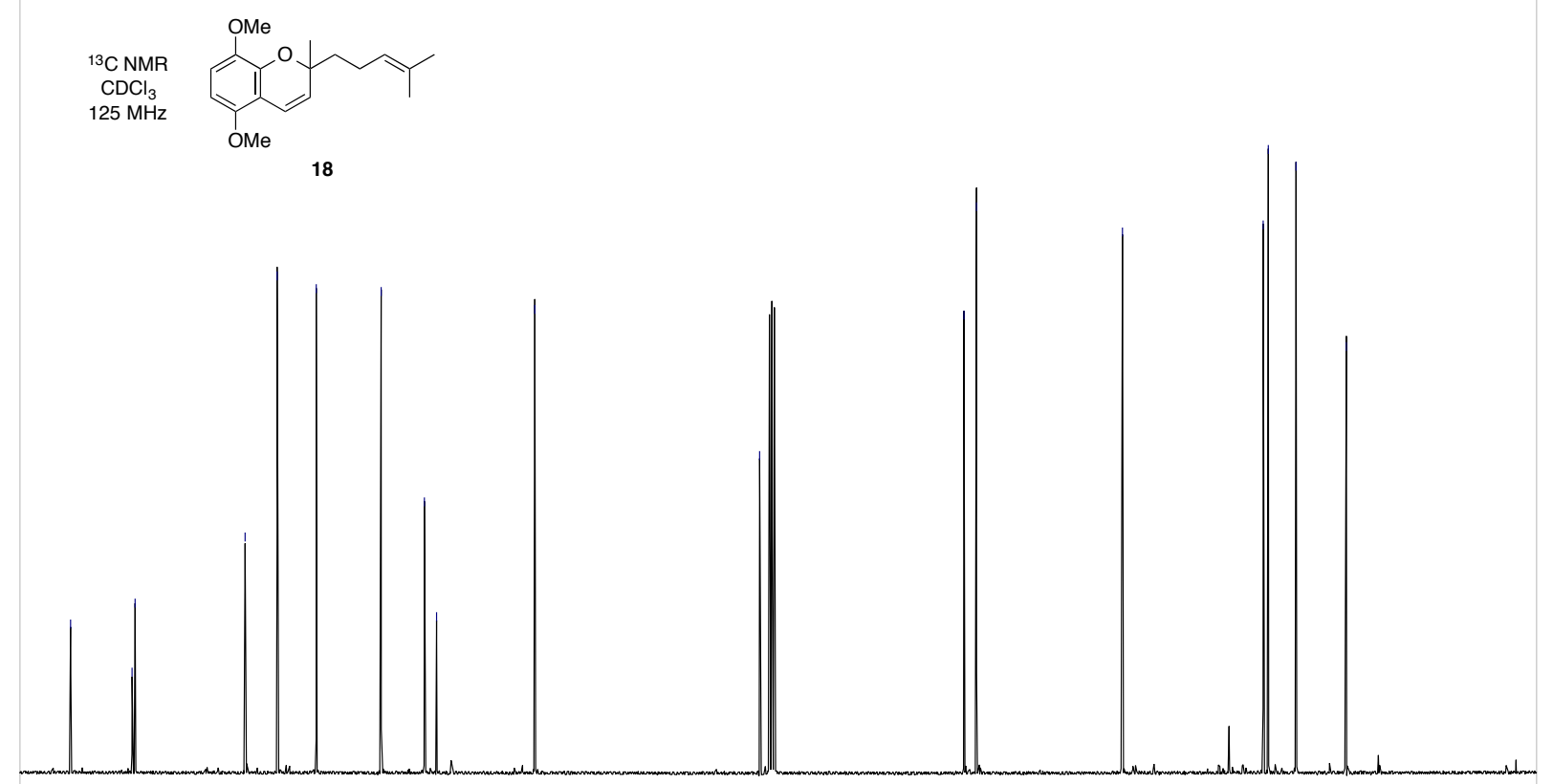

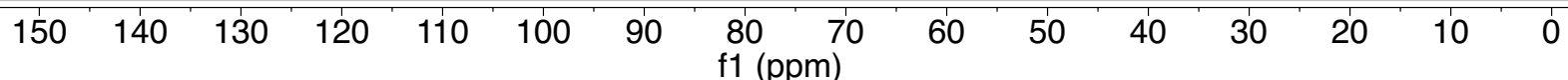



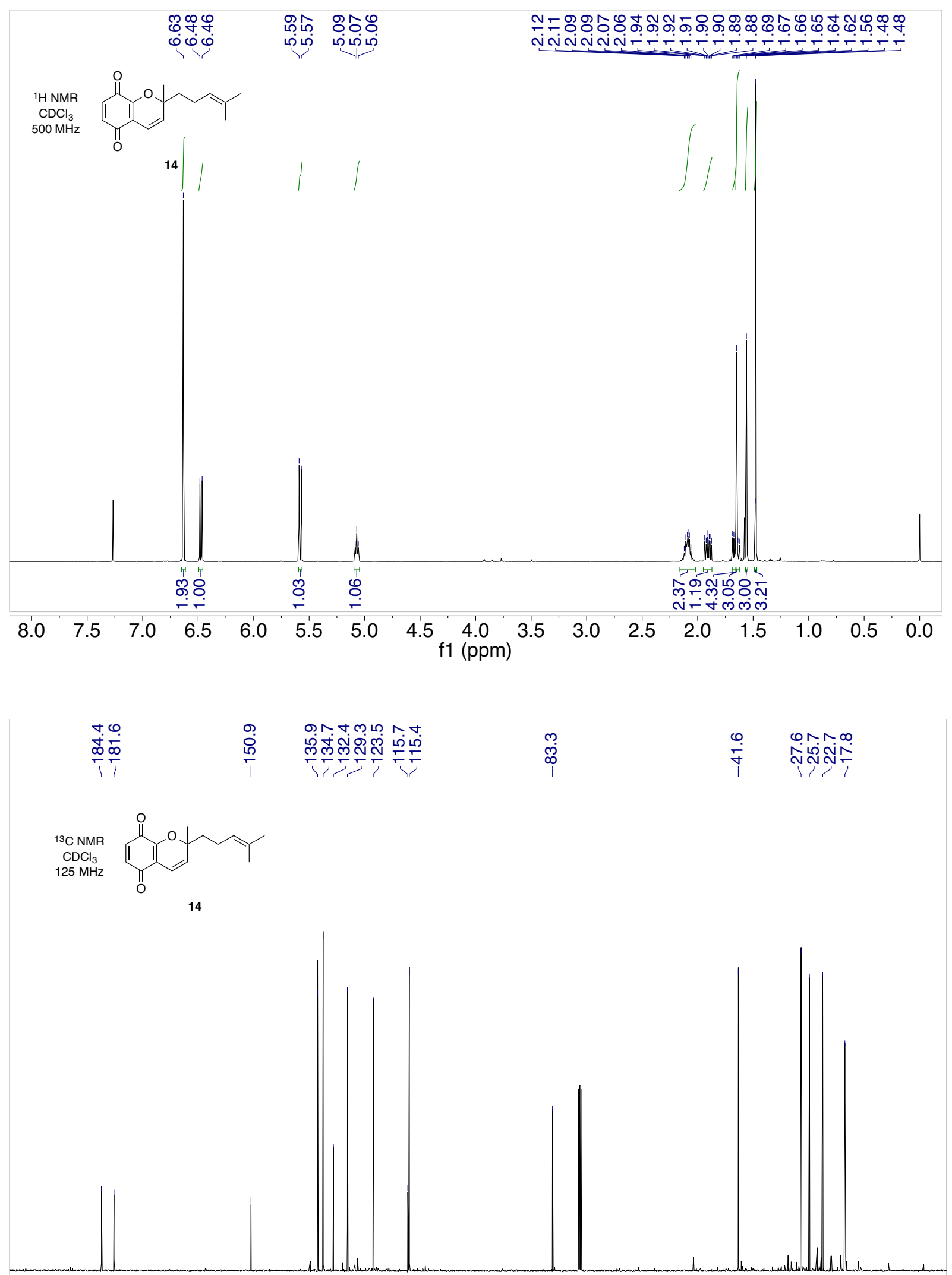

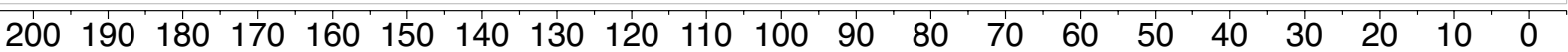
f1 (ppm) 

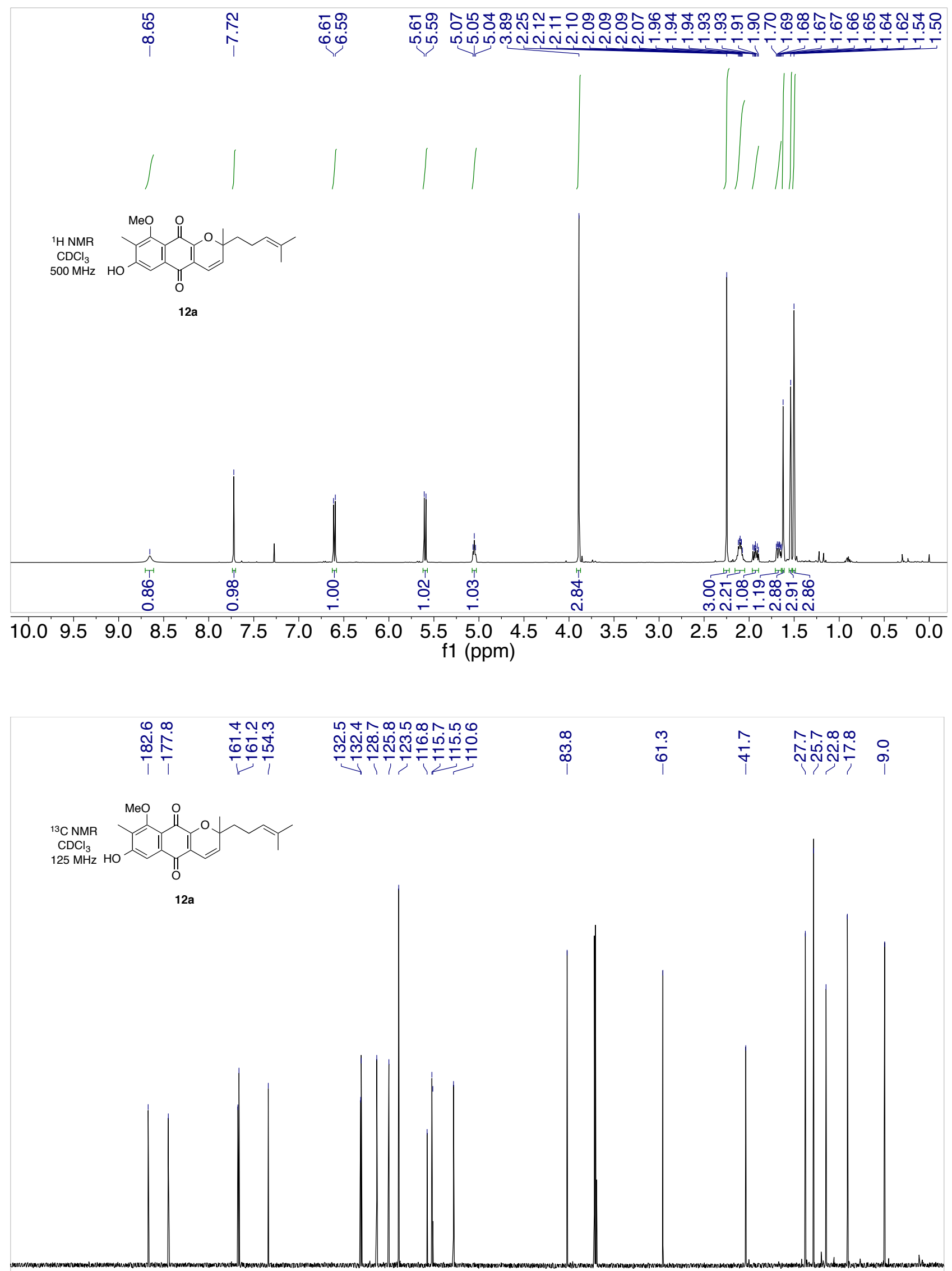

\begin{tabular}{llllllllllllllllllllllllll}
\hline 210 & 200 & 190 & 180 & 170 & 160 & 150 & 140 & 130 & 120 & 110 & 100 & 90 & 80 & 70 & 60 & 50 & 40 & 30 & 20 & 10 & 0
\end{tabular} $\mathrm{f} 1$ (ppm) 


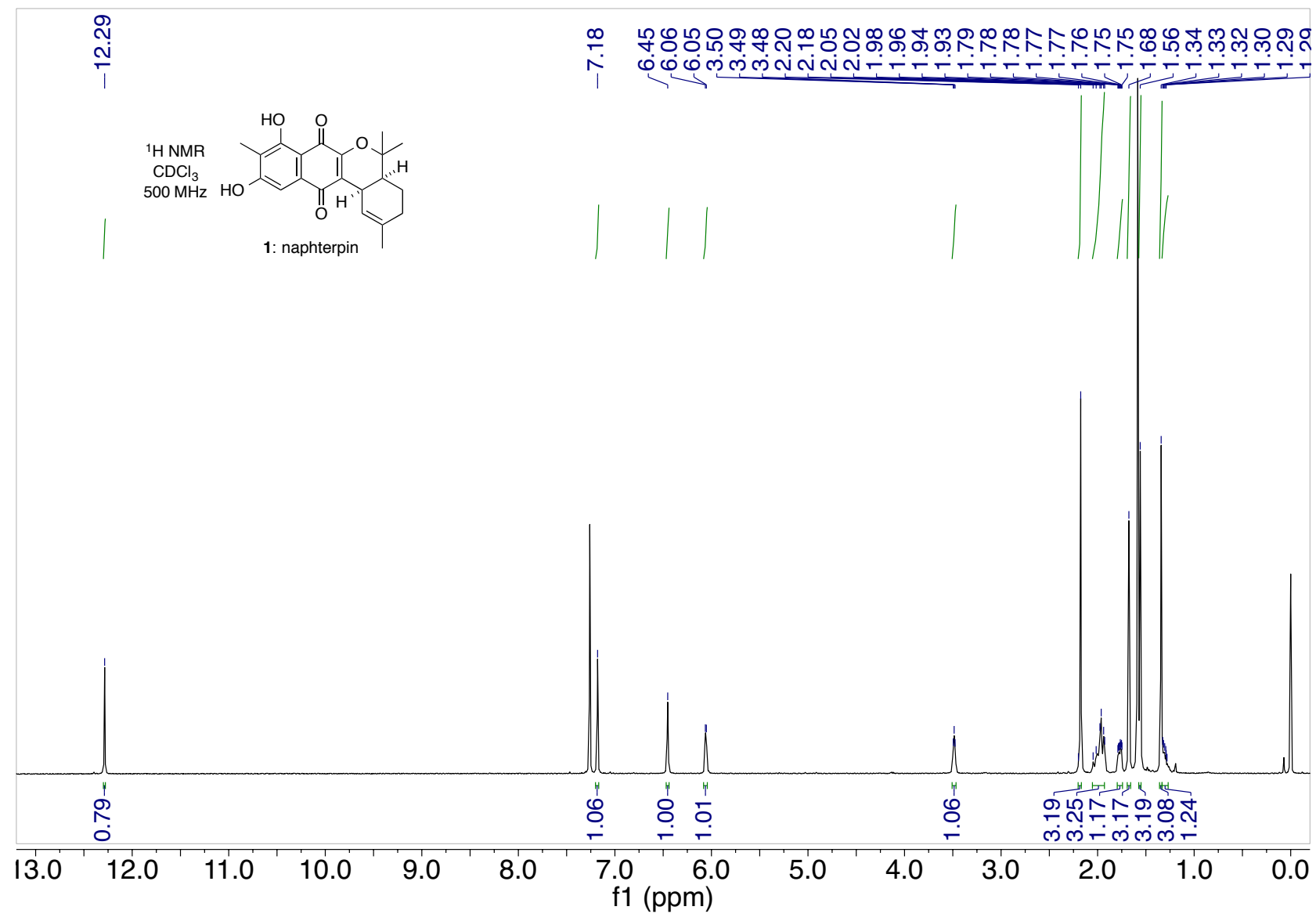

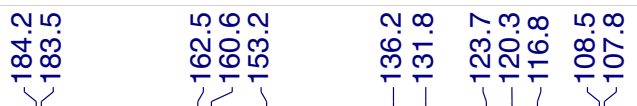

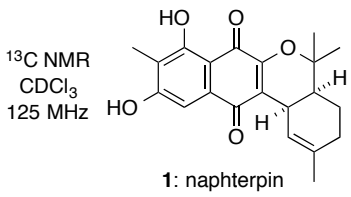

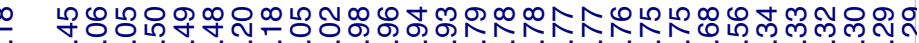

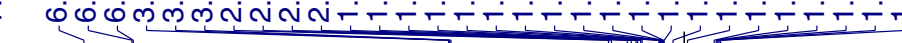
1 (ppm) 


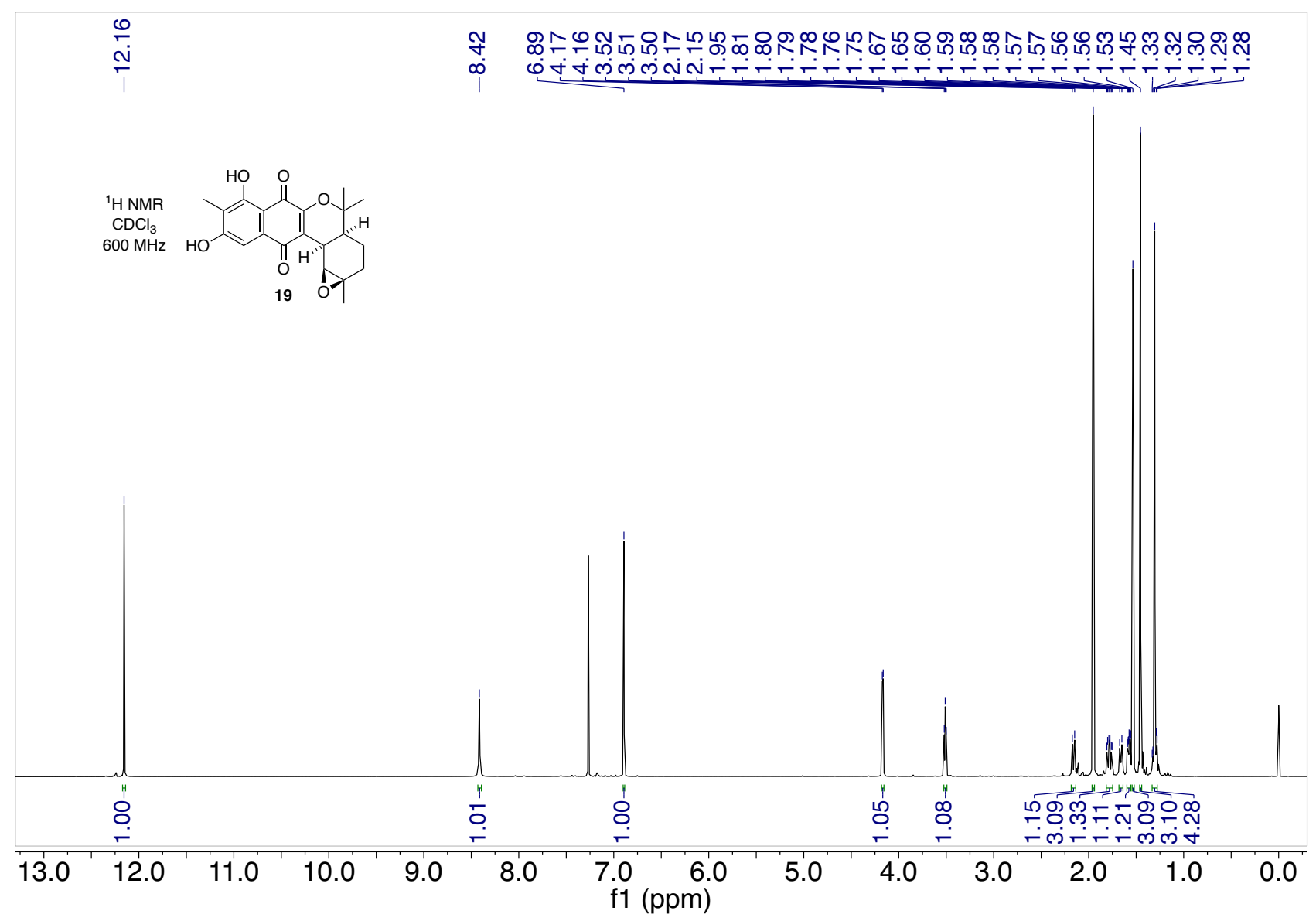

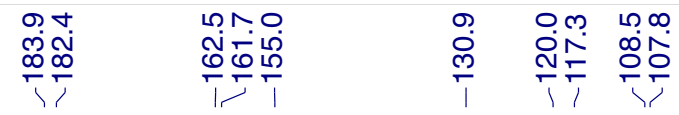

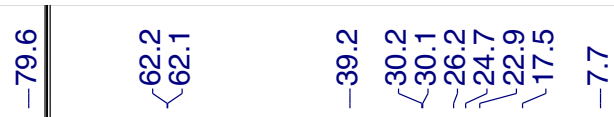

${ }_{150 \mathrm{MHz}}^{{ }^{13} \mathrm{C} \mathrm{NMRC}_{3}}$

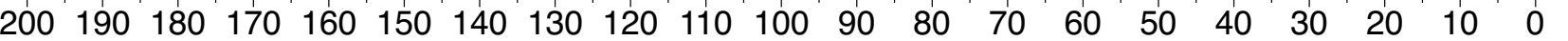
f1 (ppm) 

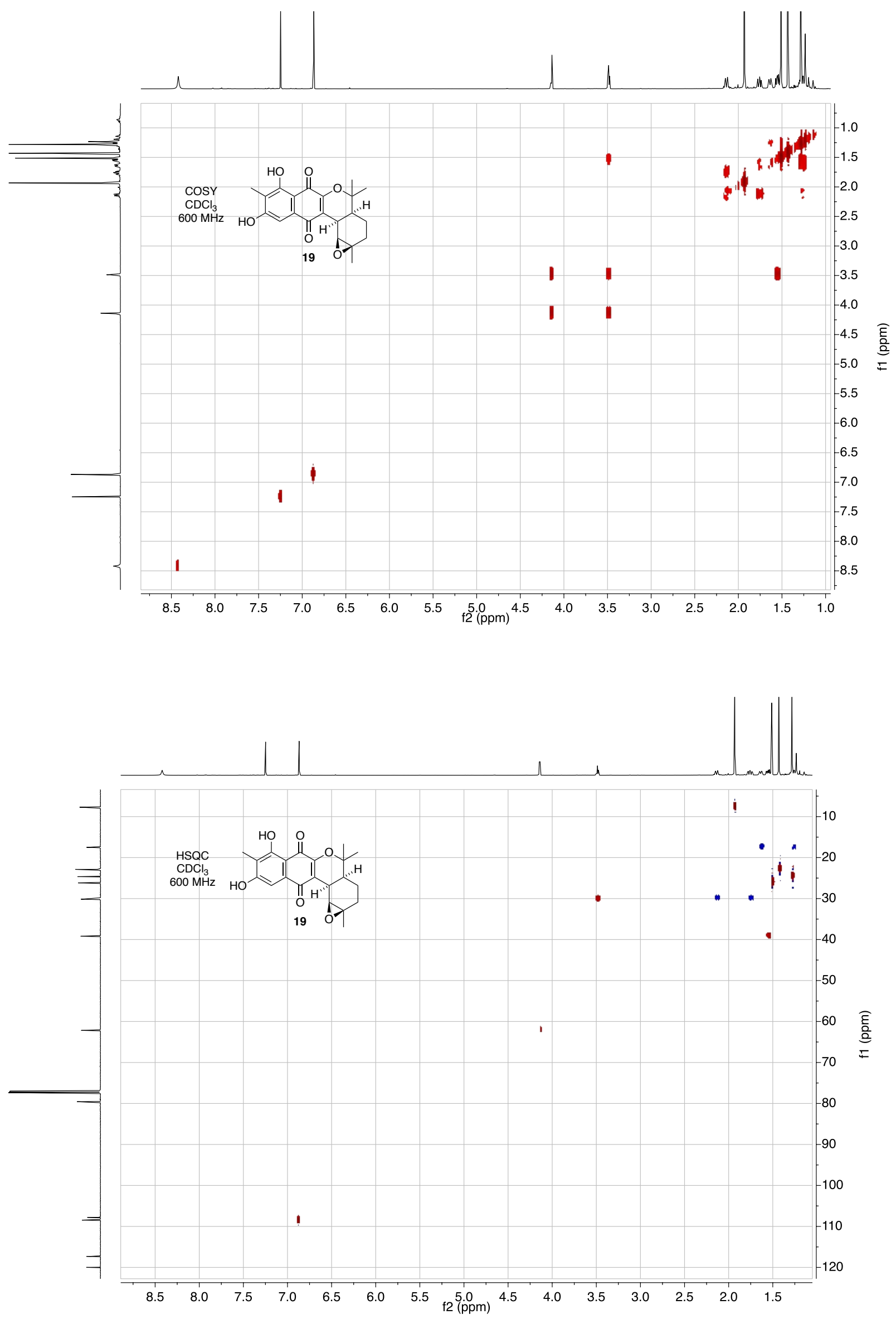

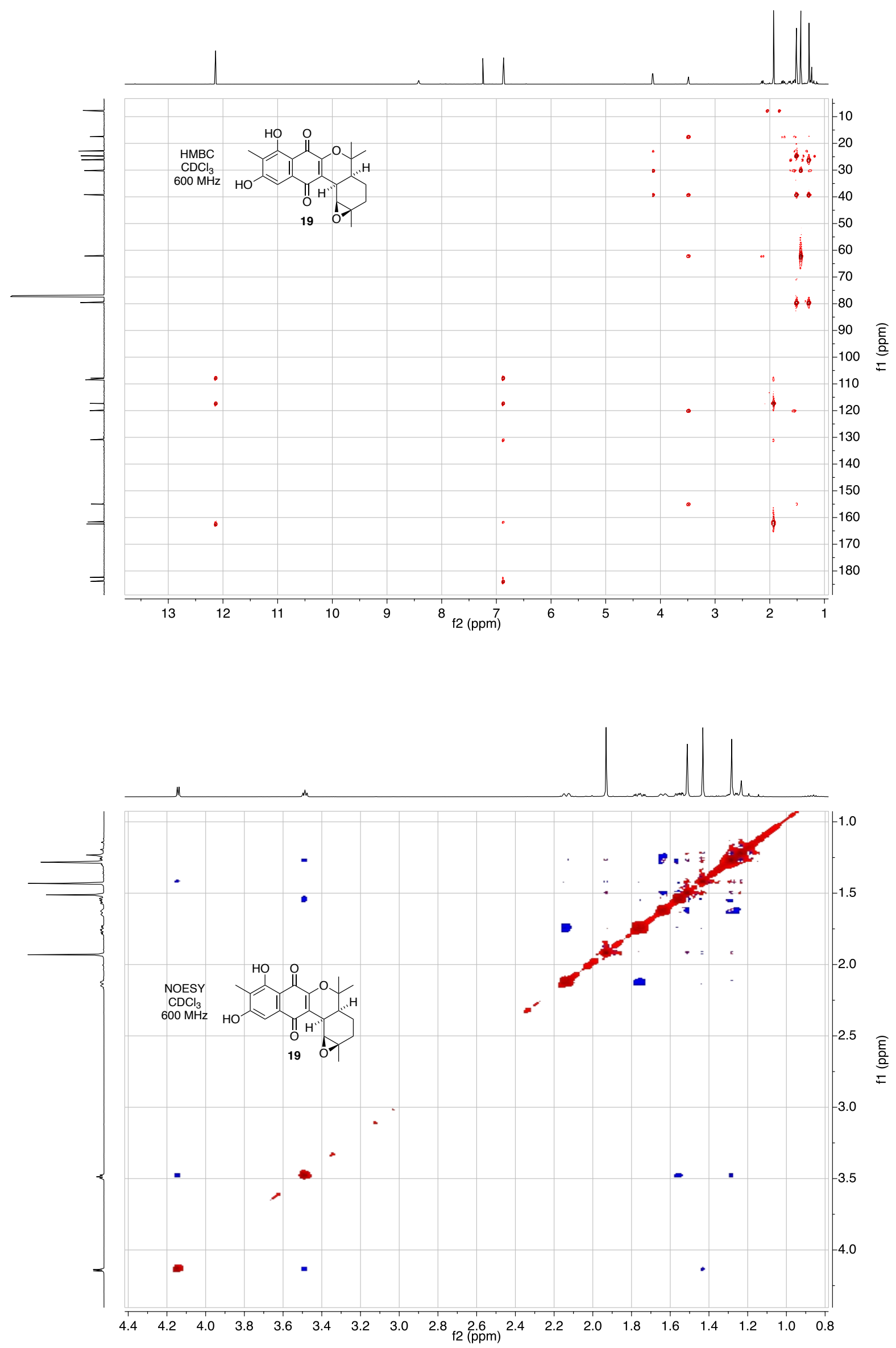

28 


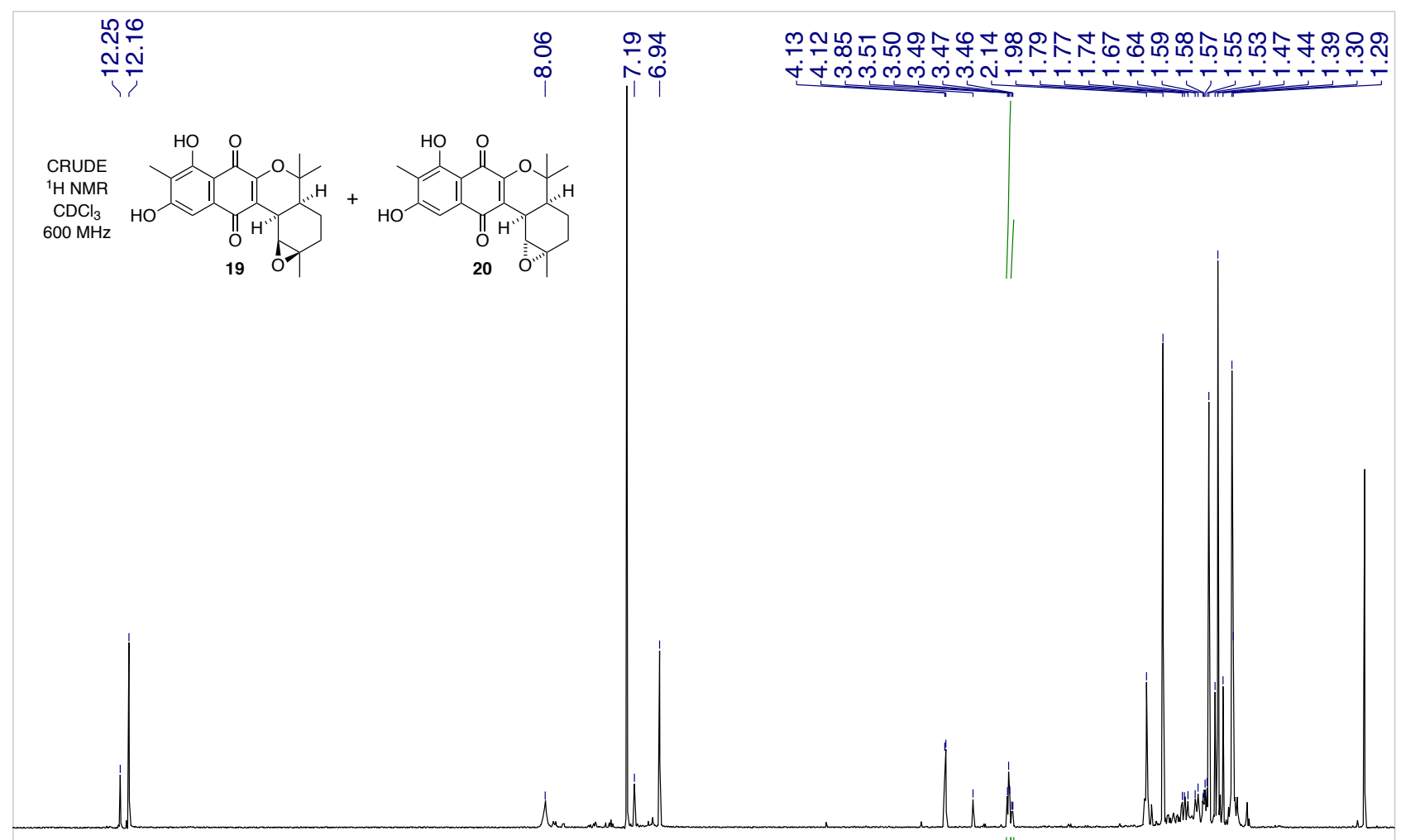

잉으
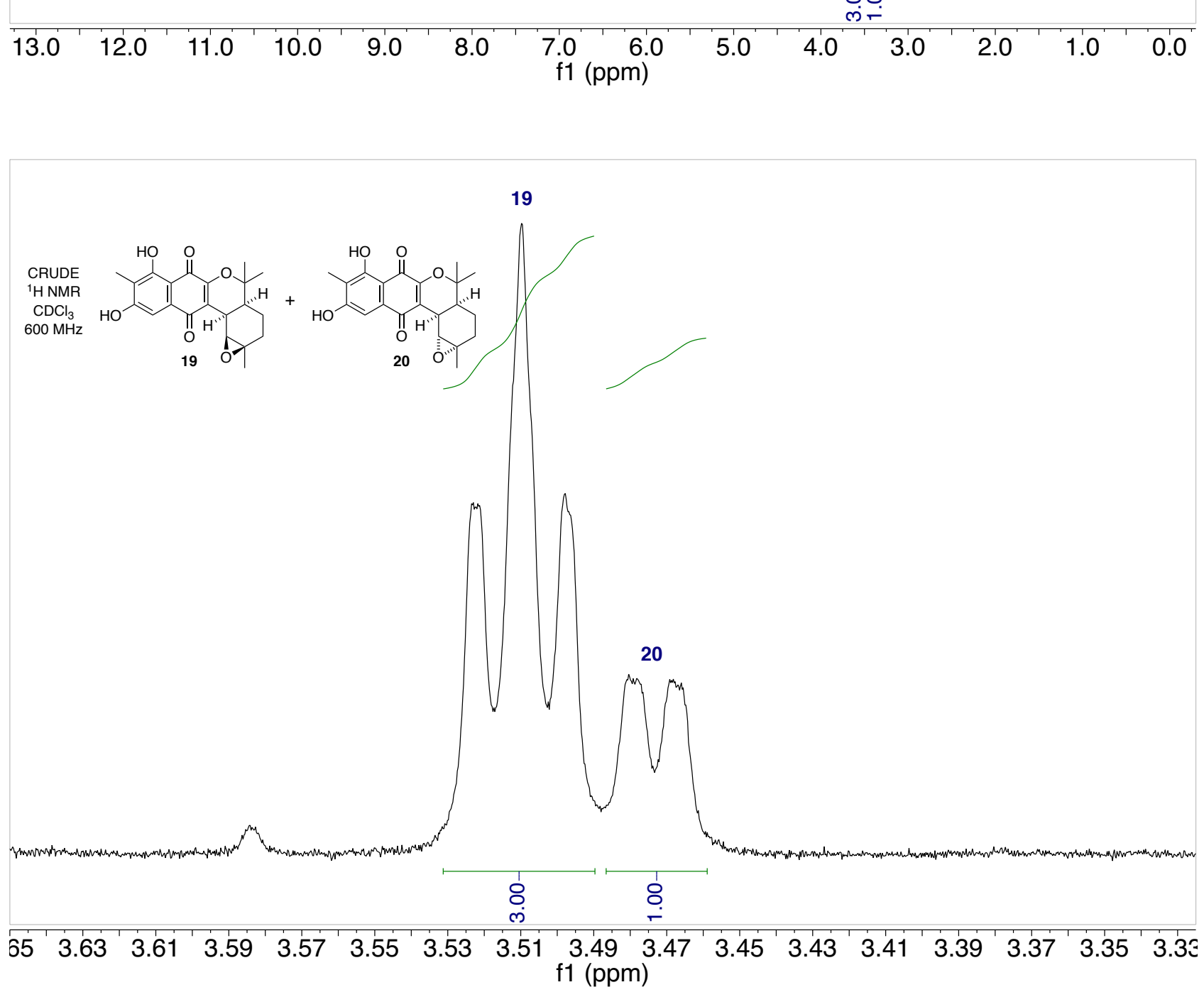

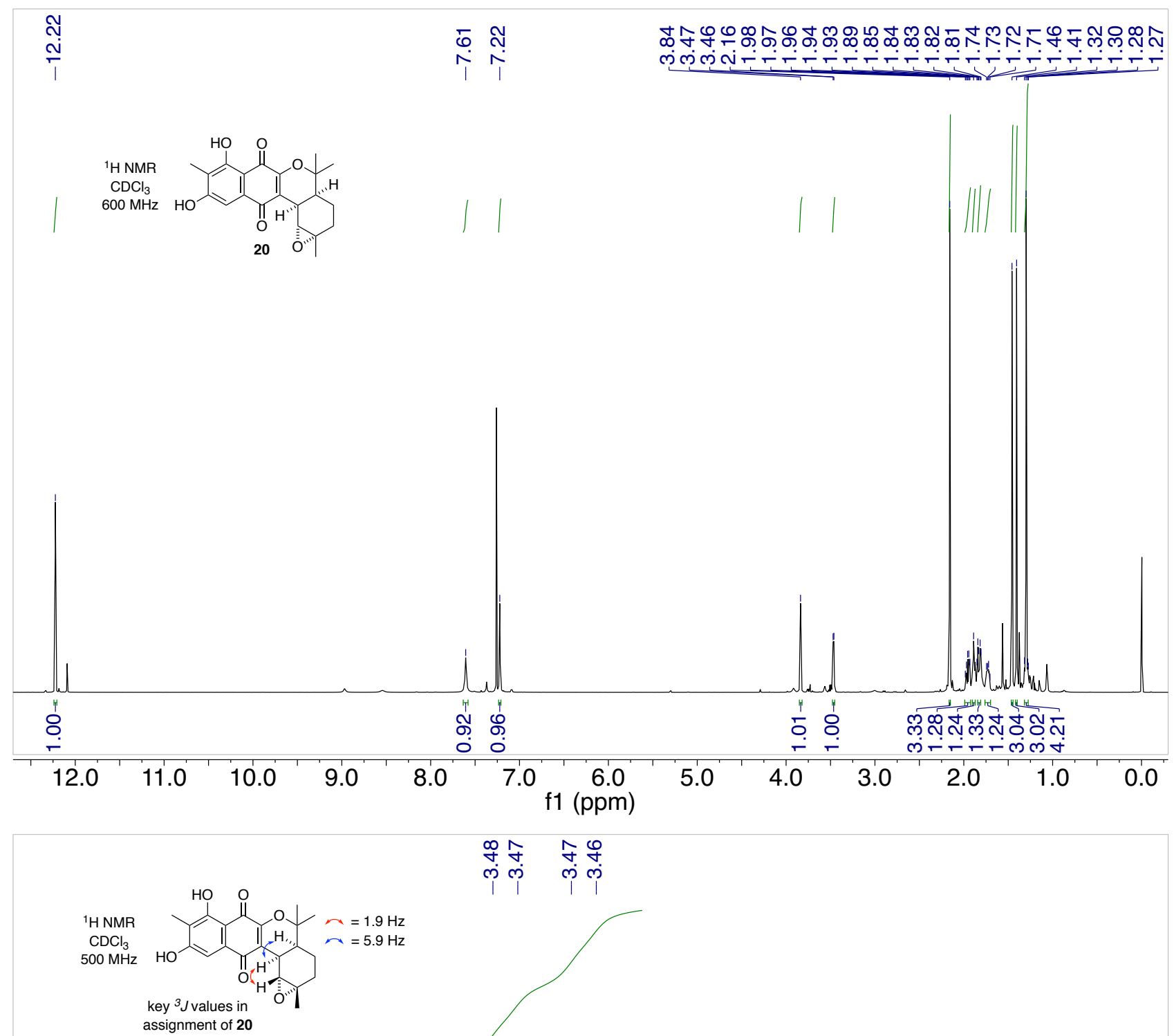

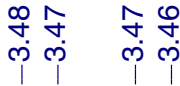

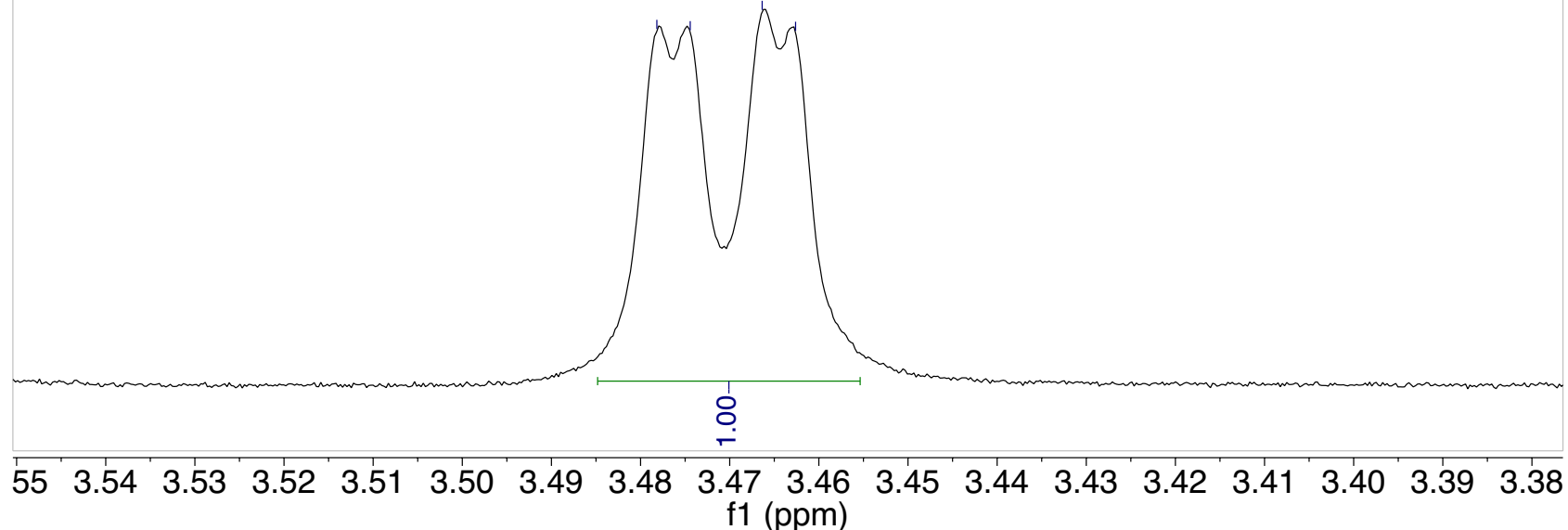




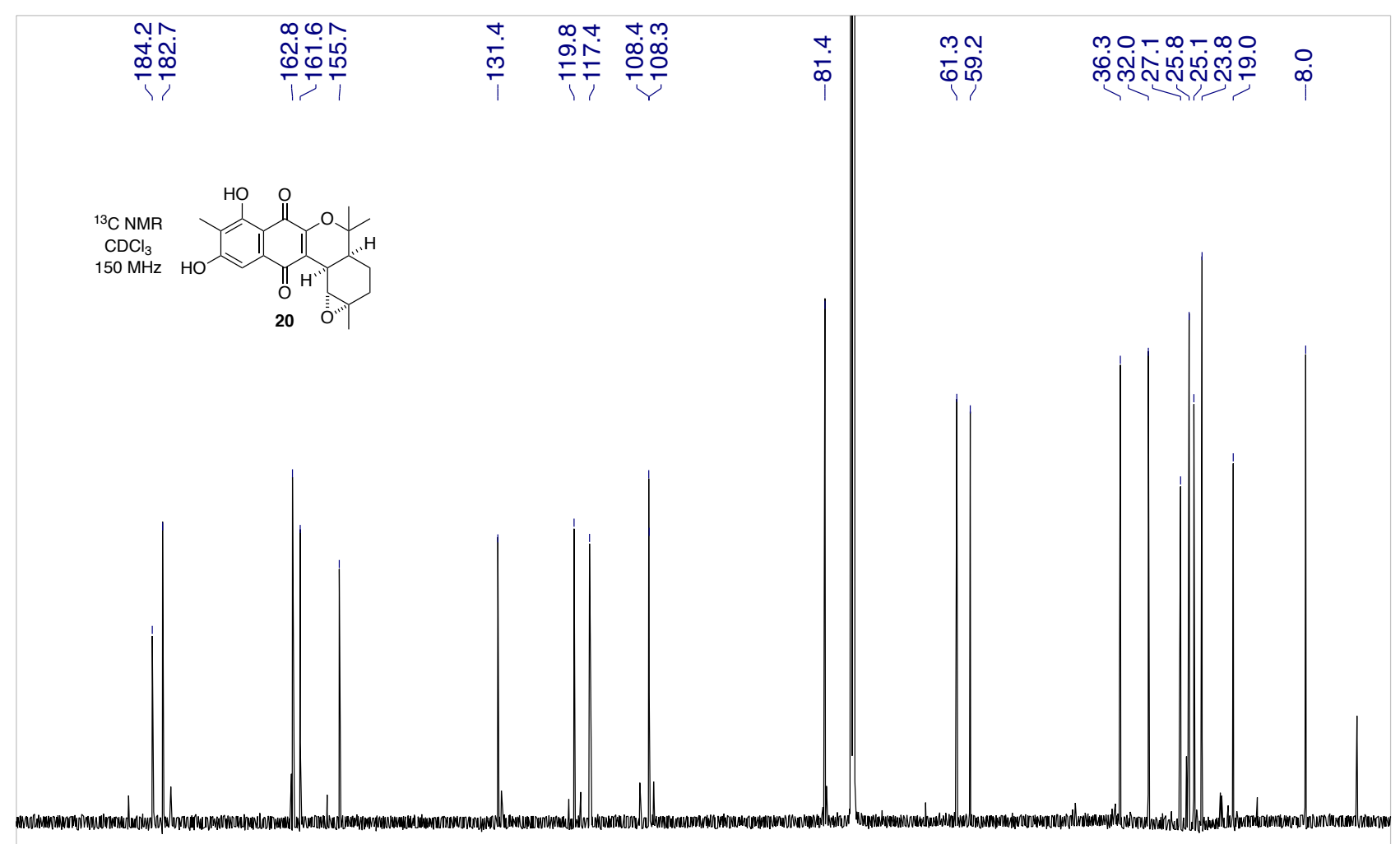

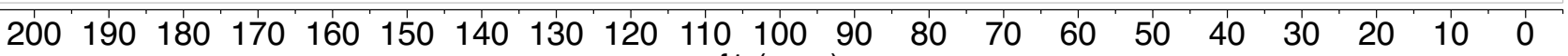
f1 (ppm)

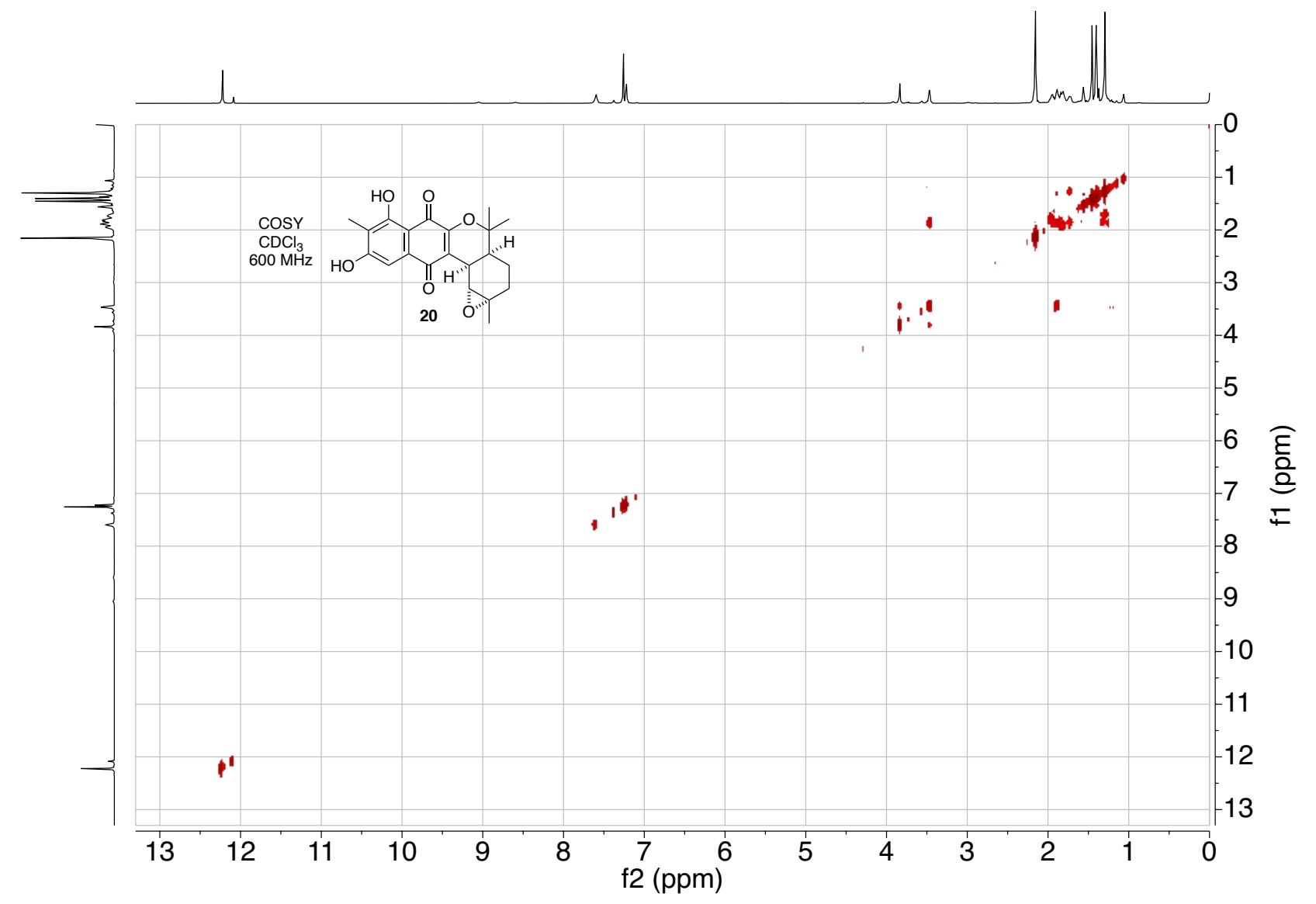



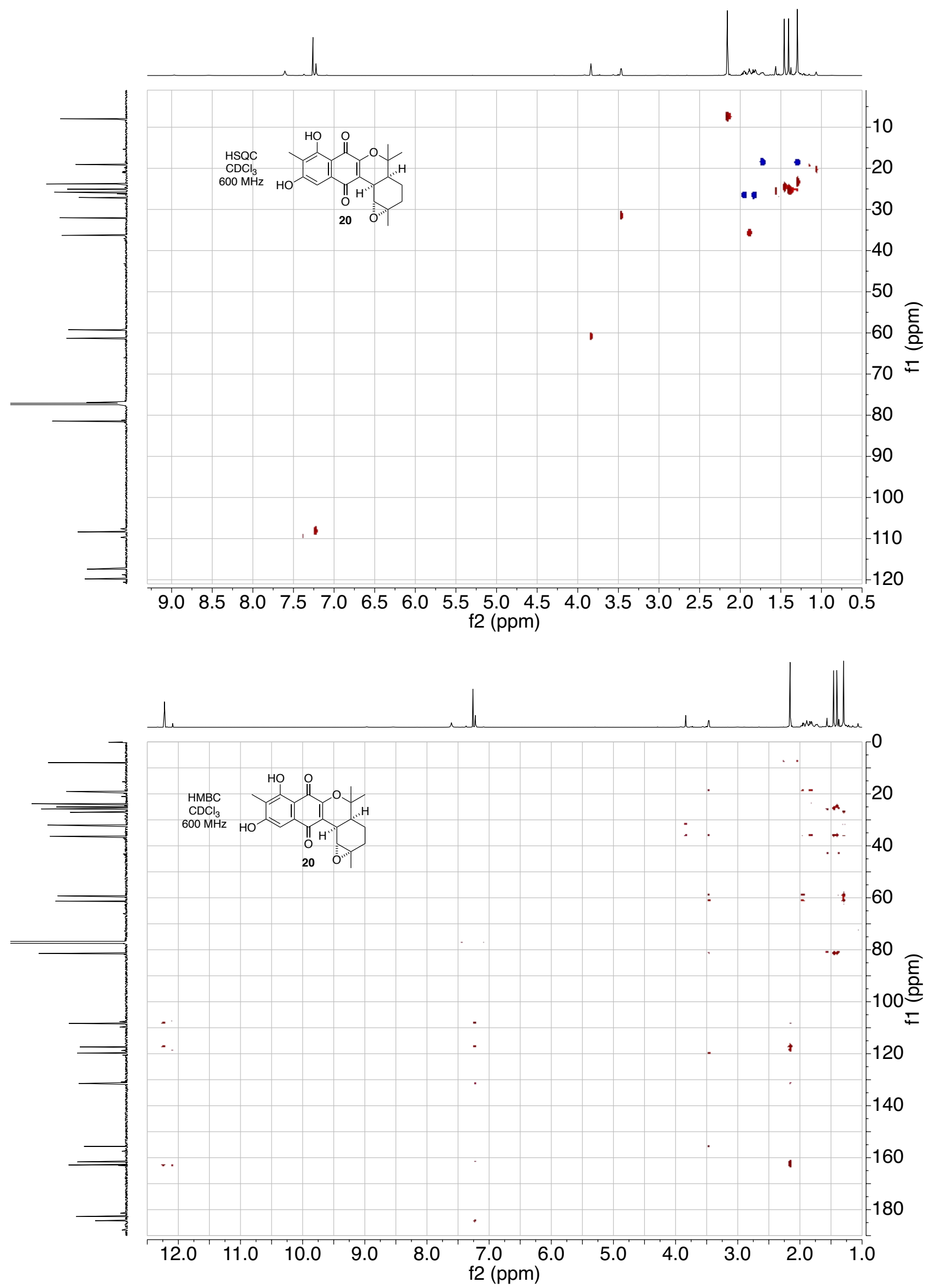


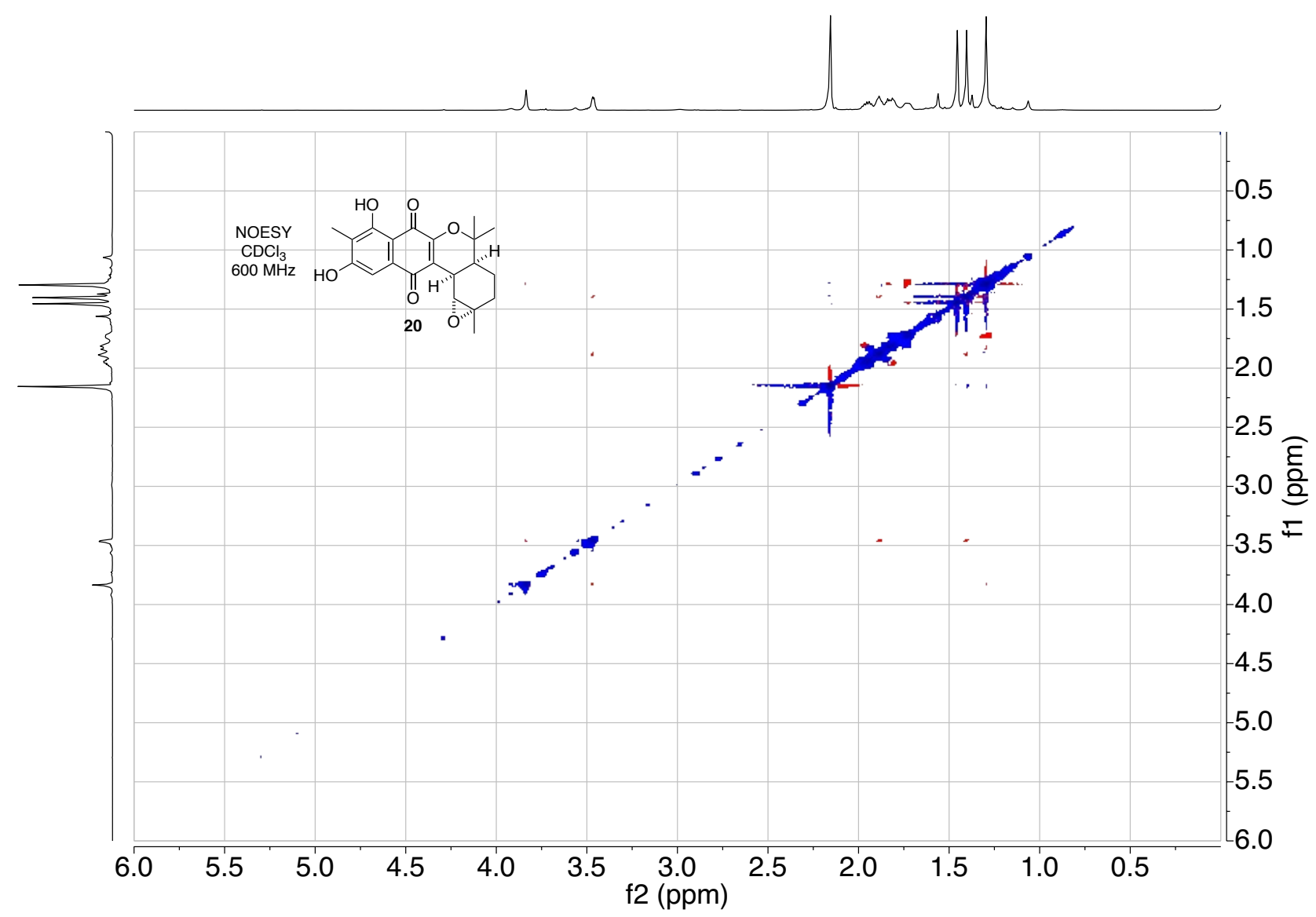



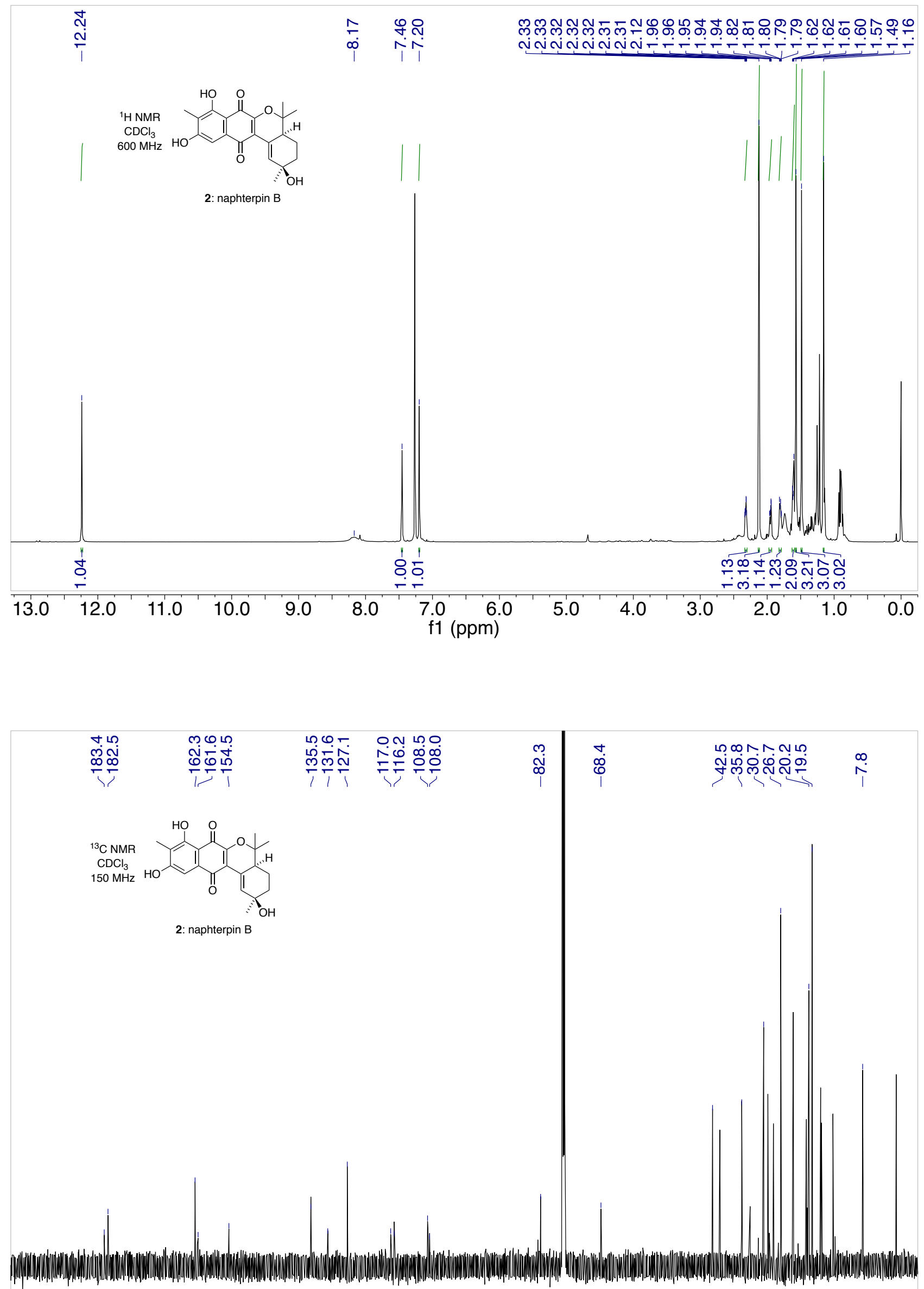

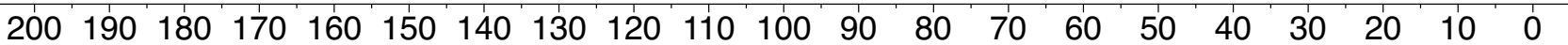
$\mathrm{f} 1(\mathrm{ppm})$ 


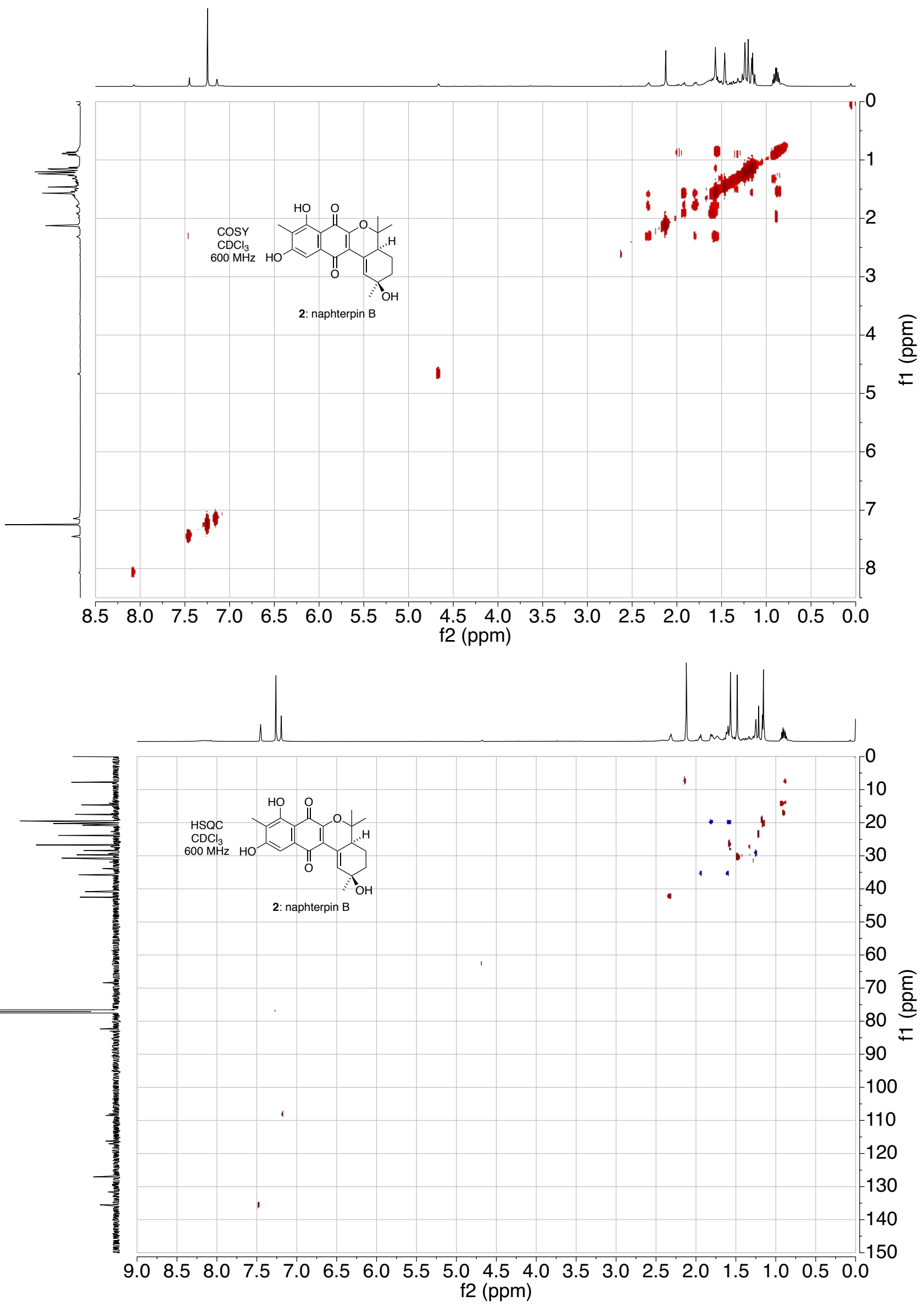




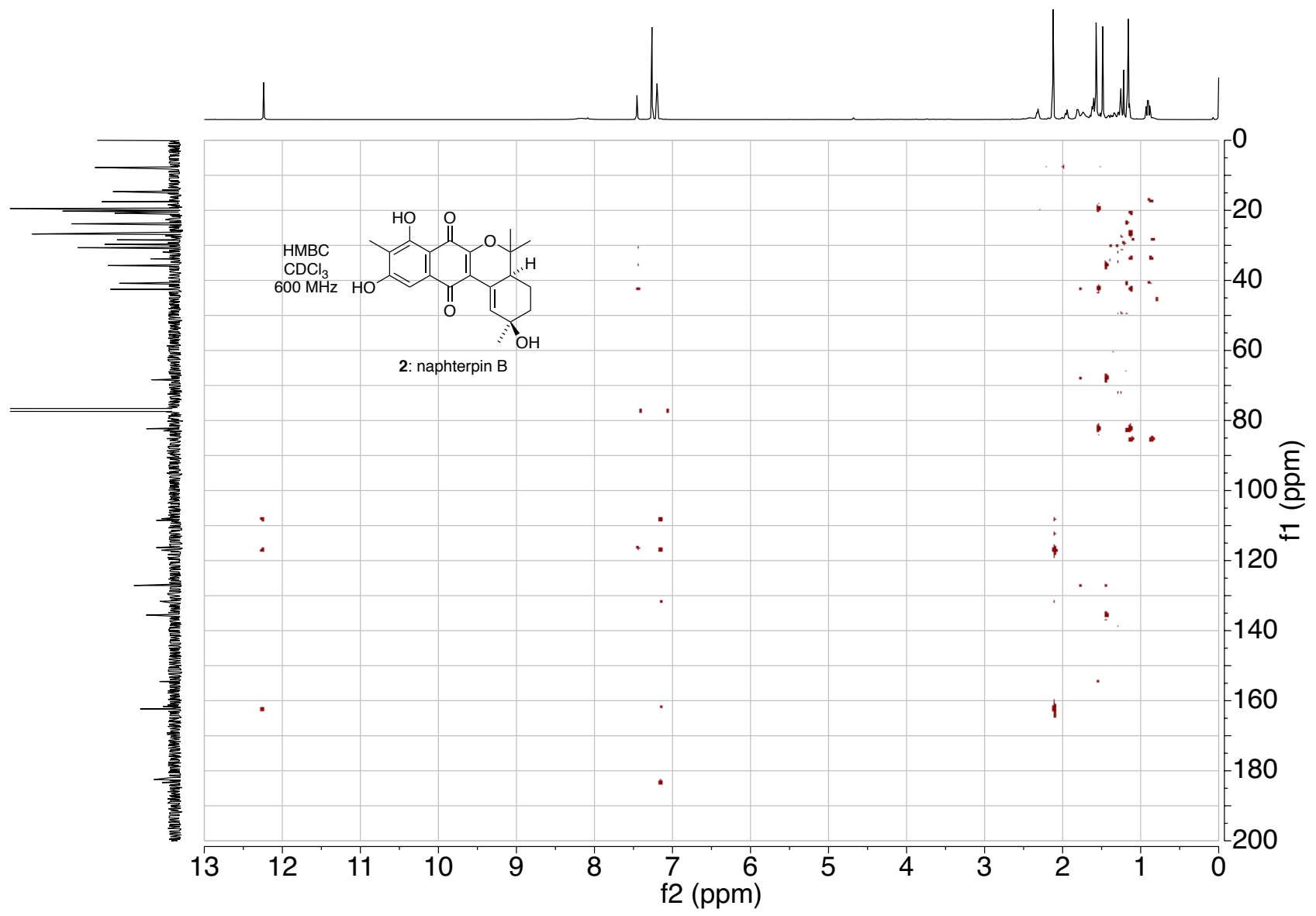

36 


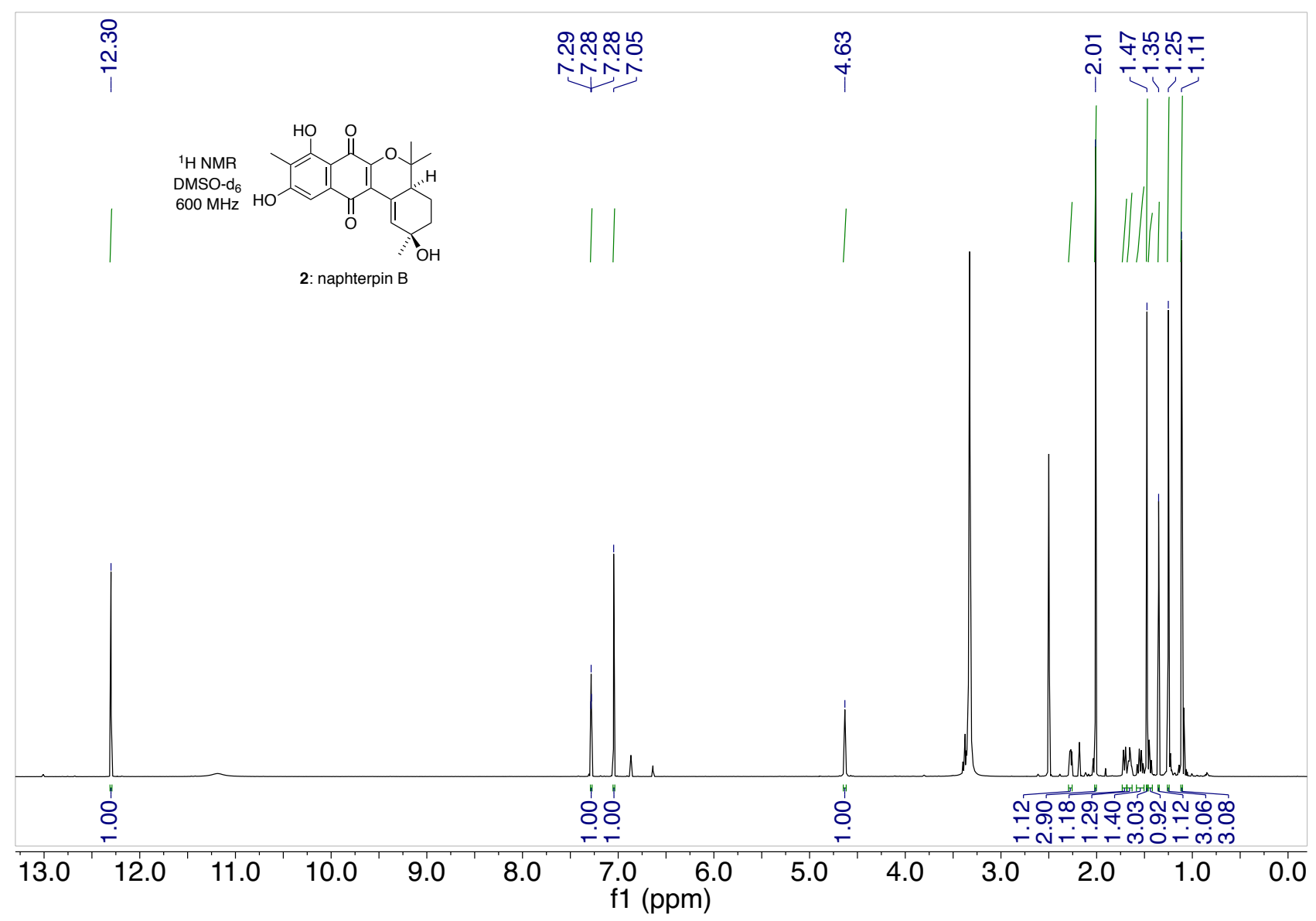

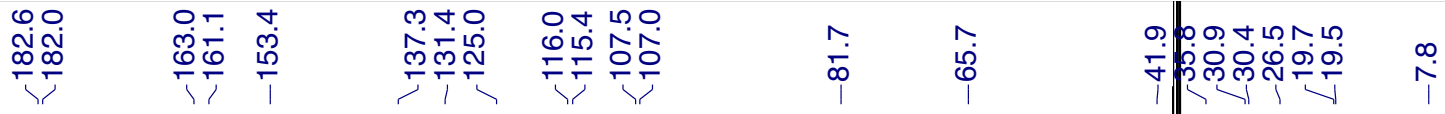

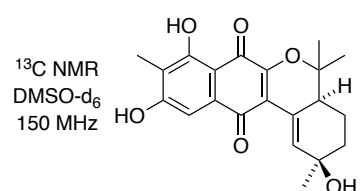

2: naphterpin B

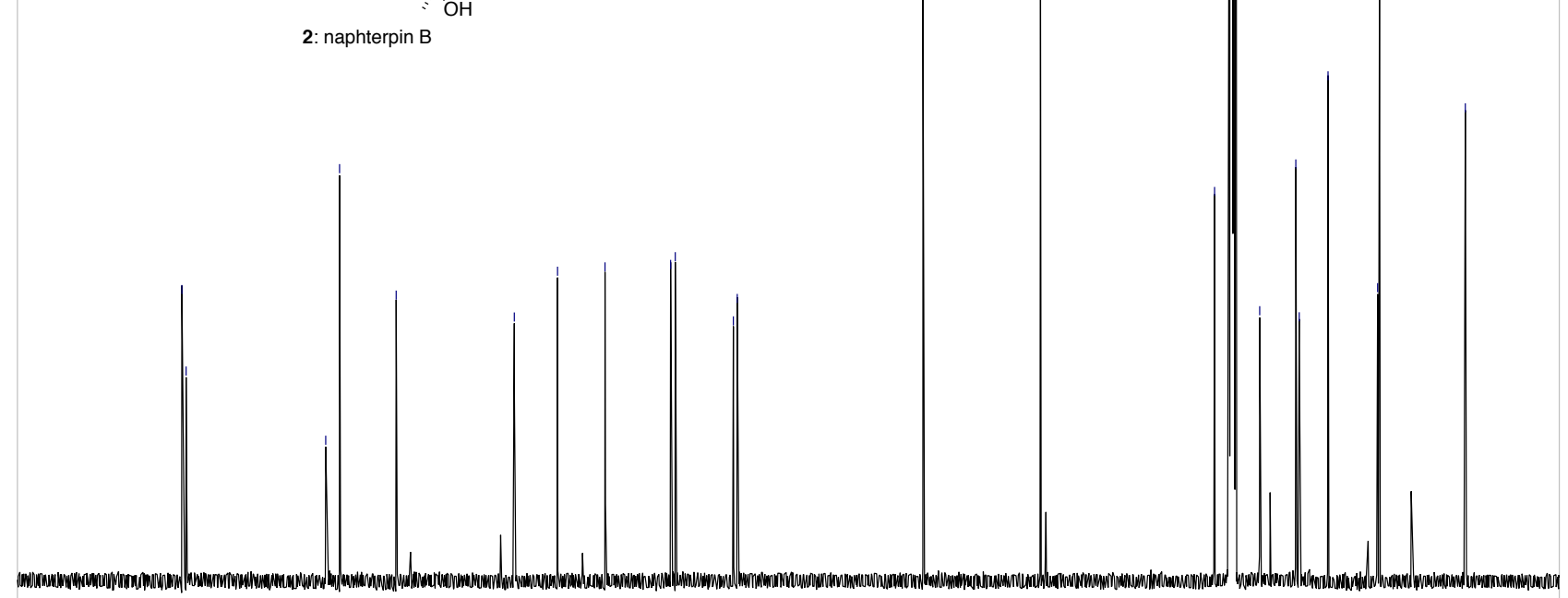

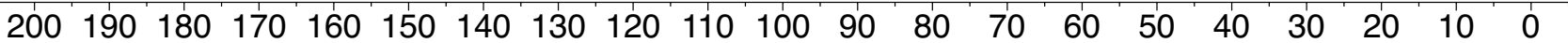
$\mathrm{f} 1(\mathrm{ppm})$ 


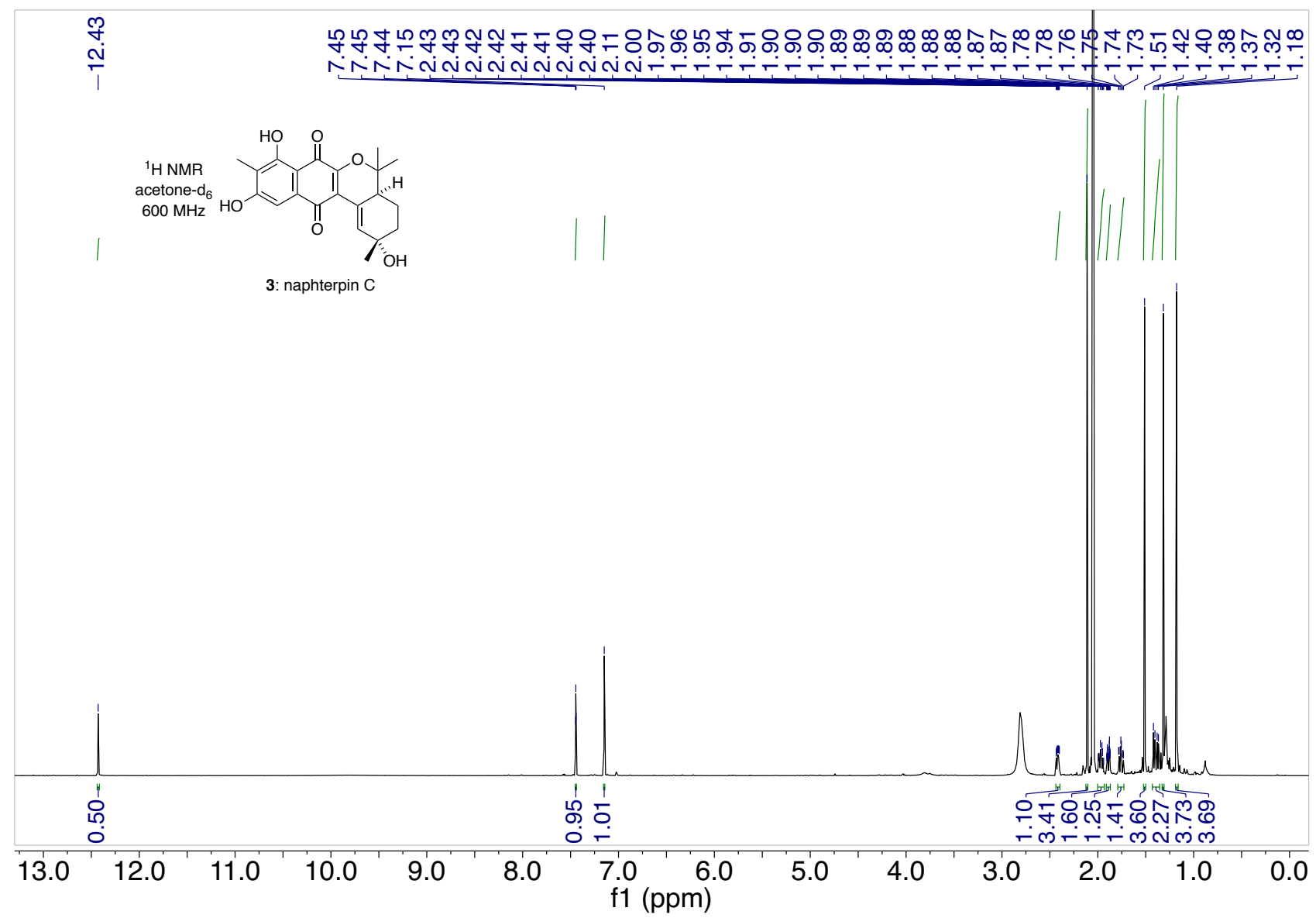

岗
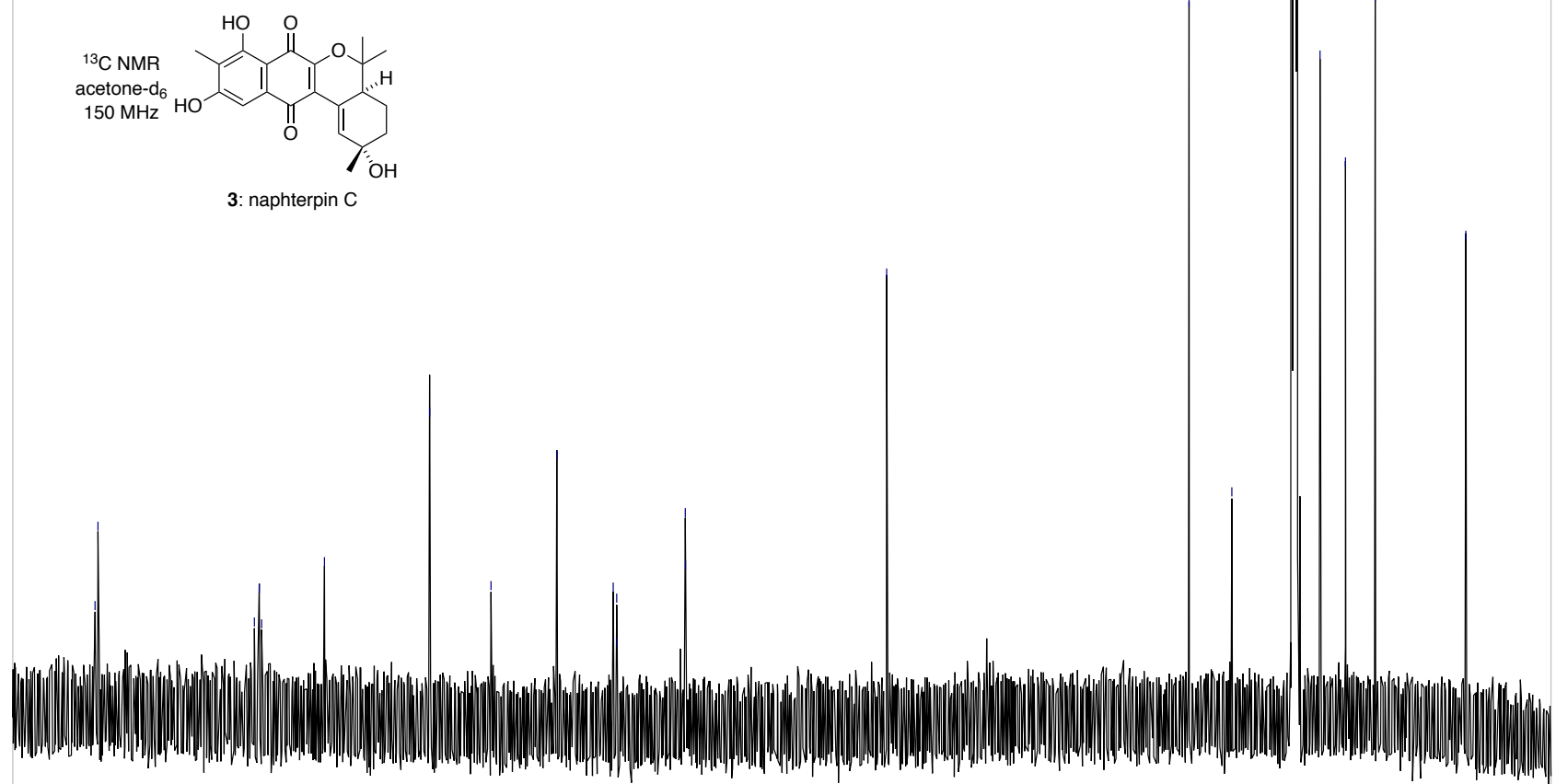

$\begin{array}{lllllllllllllllllllllll}190 & 180 & 170 & 160 & 150 & 140 & 130 & 120 & 110 & 100 & 90 & 80 & 70 & 60 & 50 & 40 & 30 & 20 & 10 & 0 \\ \mathrm{f} 1(\mathrm{ppm})\end{array}$ 

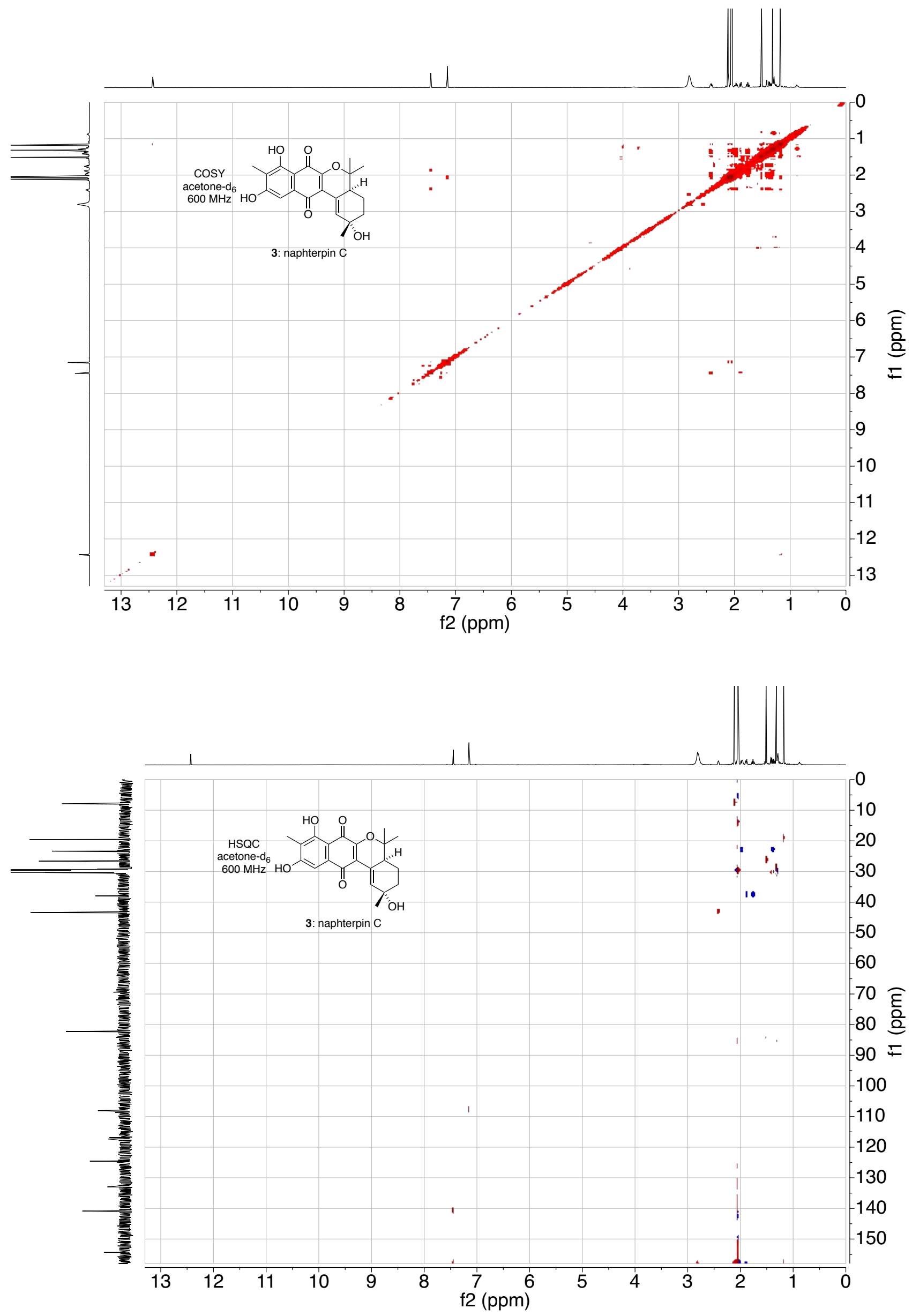

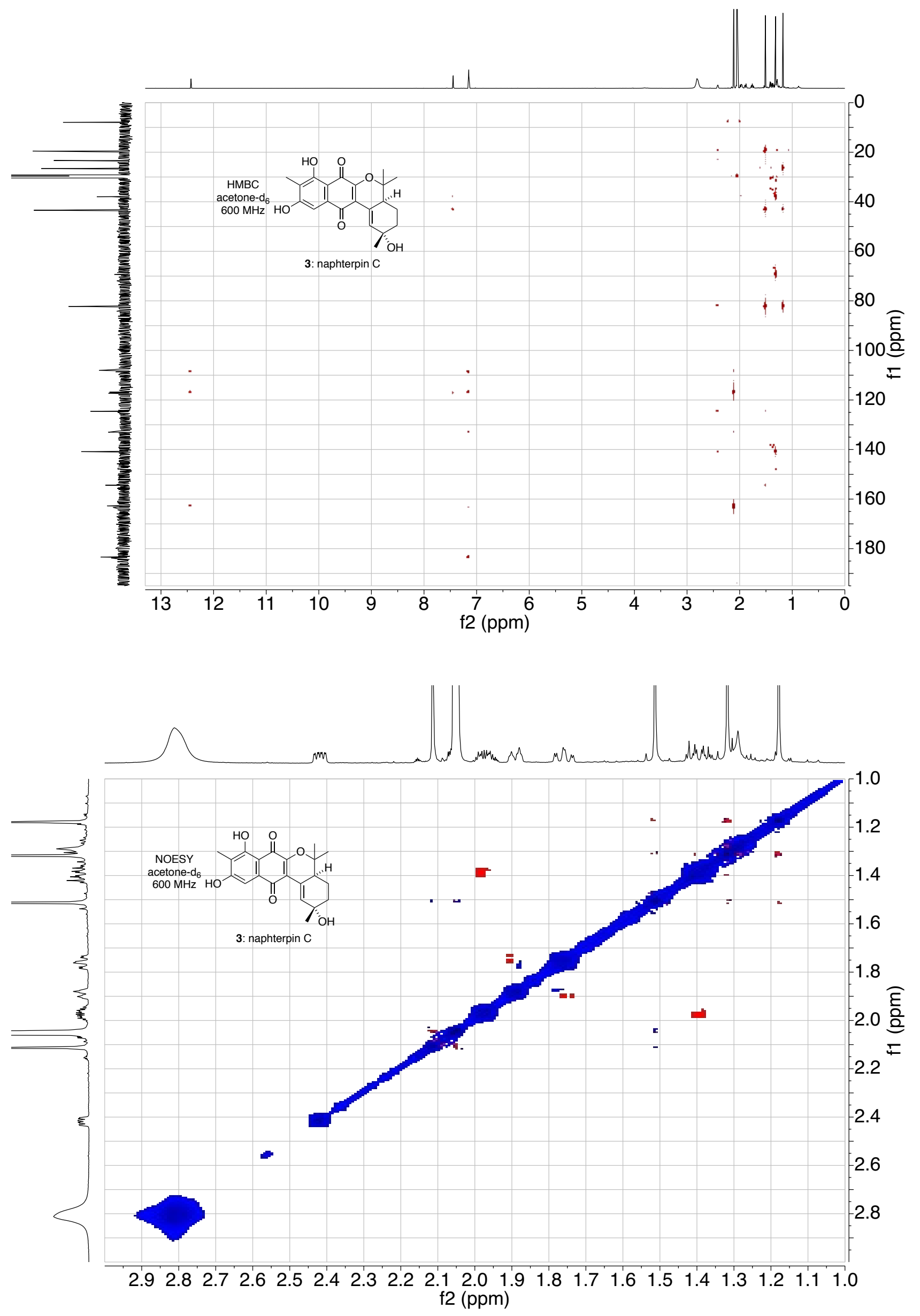


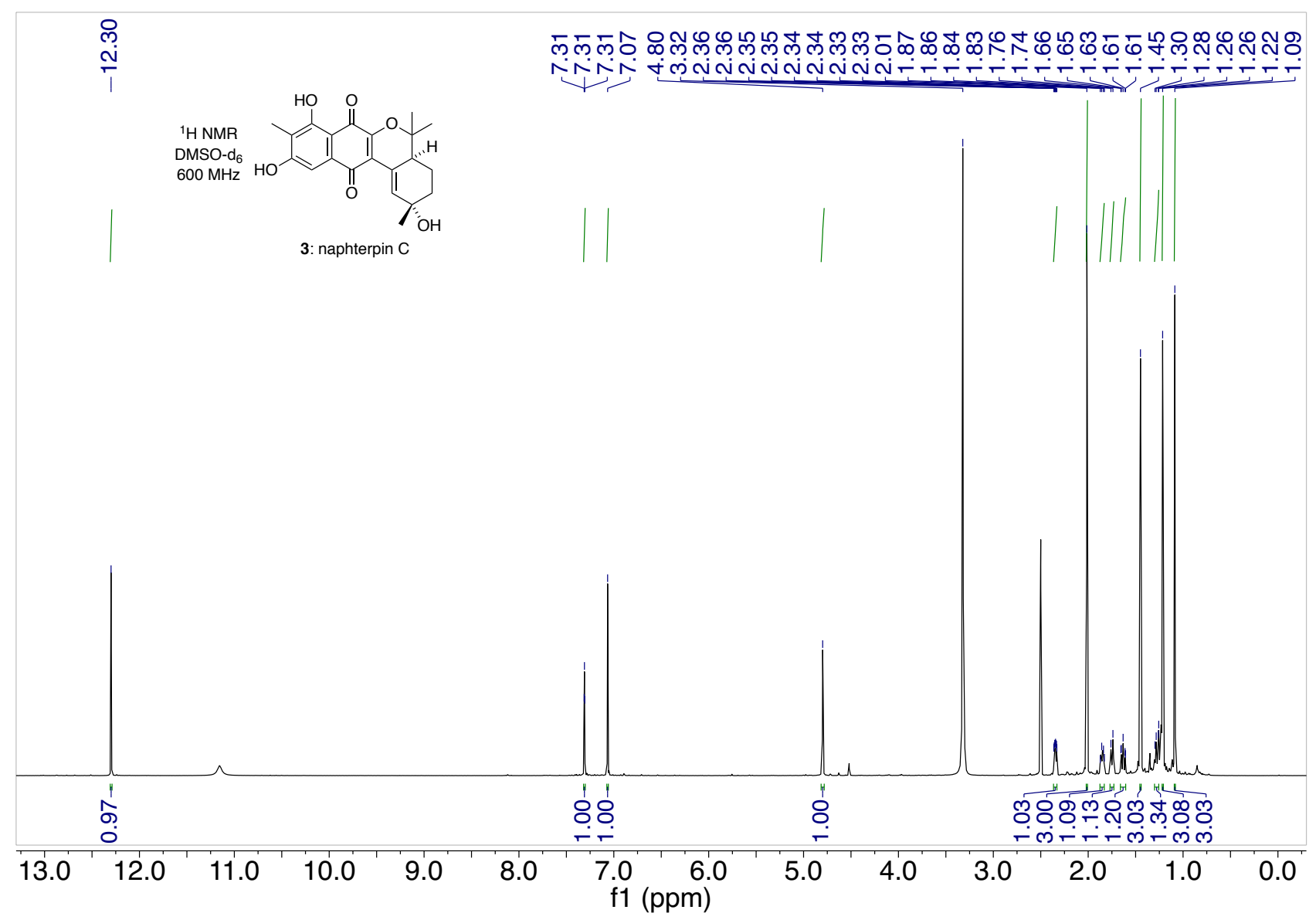

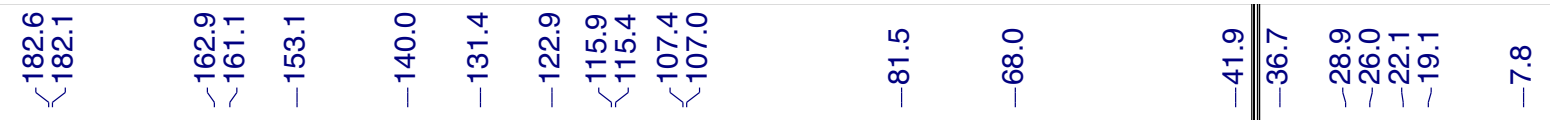
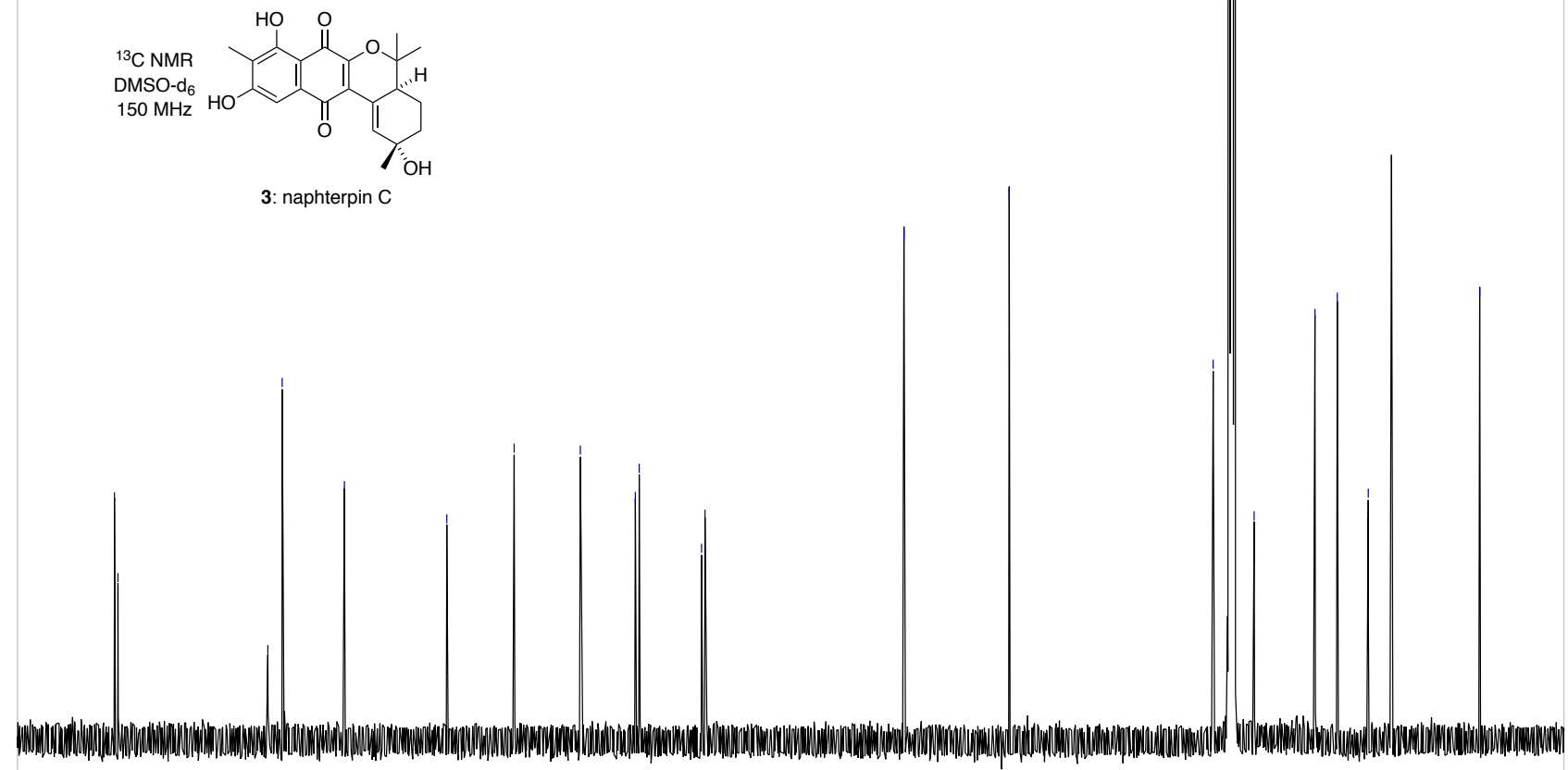

$\begin{array}{lllllllllllllllllllll}190 & 180 & 170 & 160 & 150 & 140 & 130 & 120 & 110 & 100 & 90 & 80 & 70 & 60 & 50 & 40 & 30 & 20 & 10 & 0\end{array}$ 

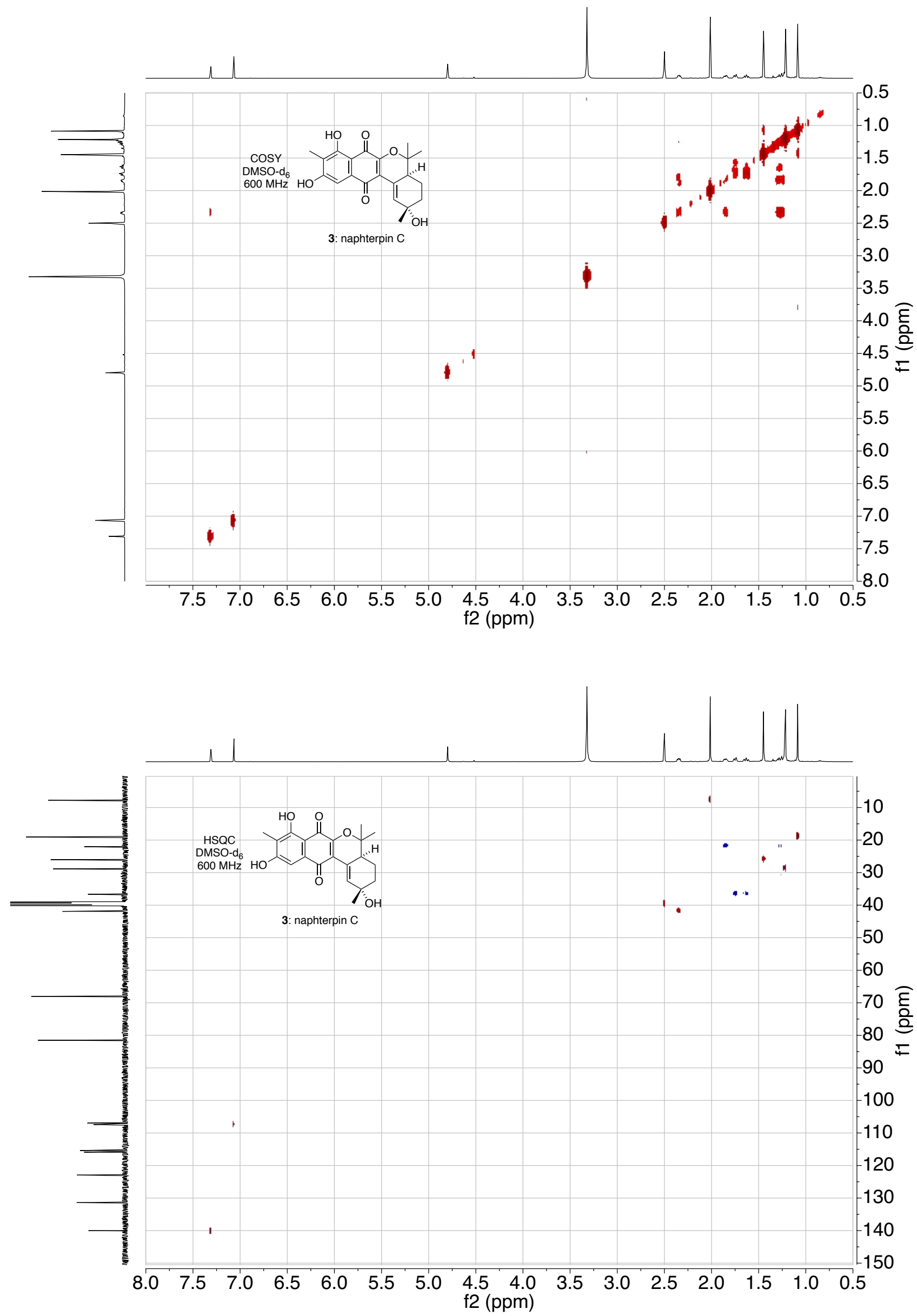

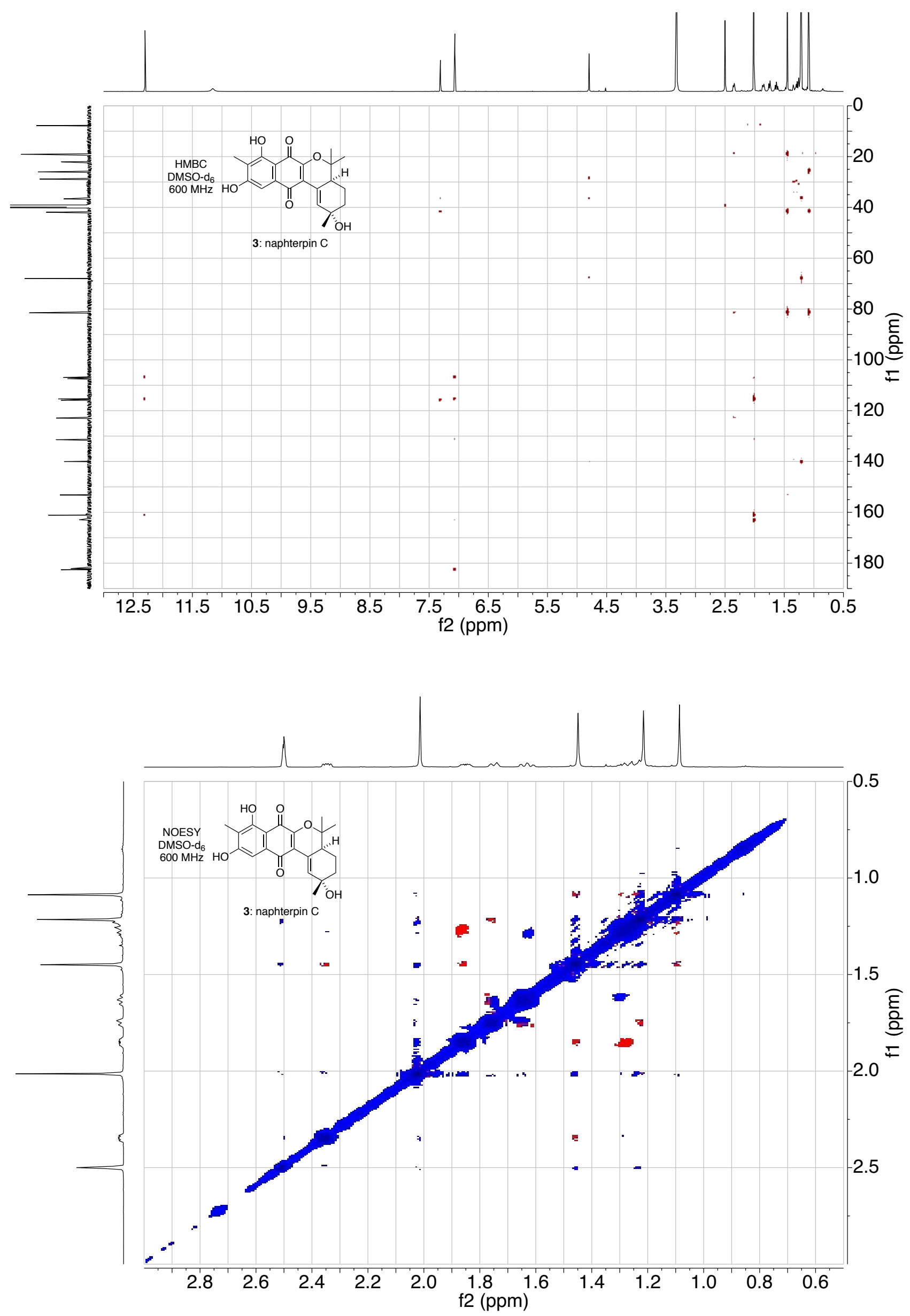


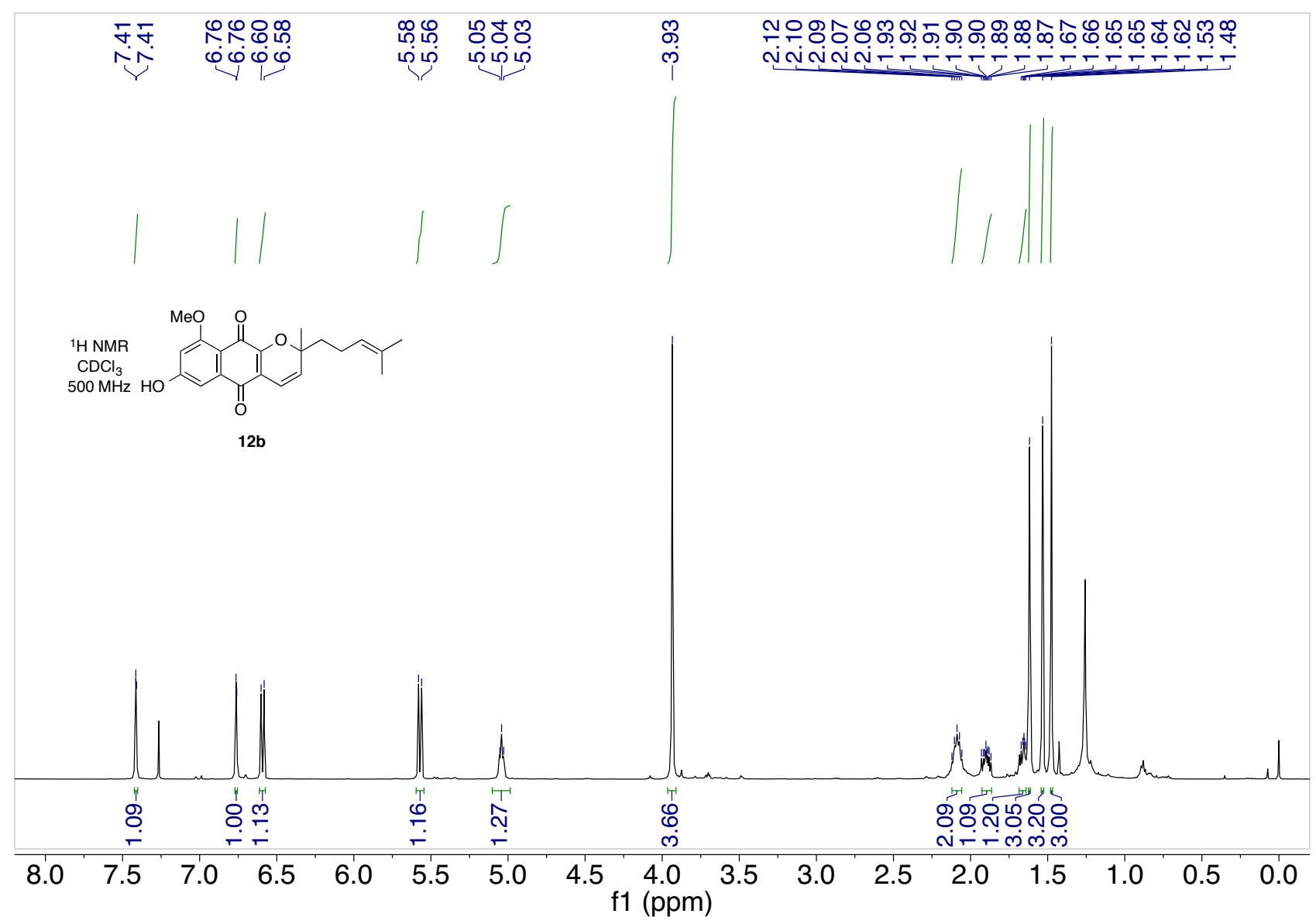

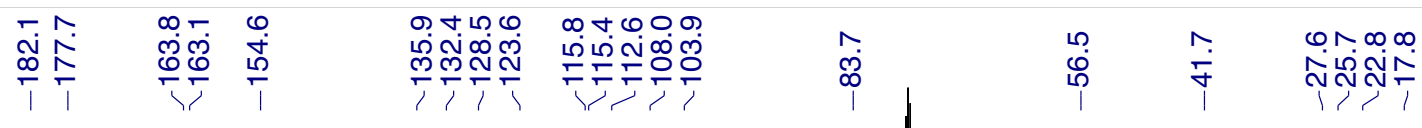

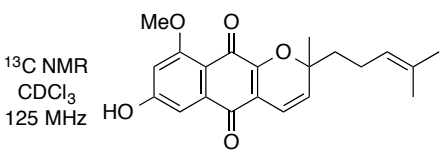

$12 b$ $\begin{array}{lllllllllllllllllllll}190 & 180 & 170 & 160 & 150 & 140 & 130 & 120 & 110 & \begin{array}{l}100 \\ \mathrm{f} 1(\mathrm{ppm})\end{array} & 80 & 70 & 60 & 50 & 40 & 30 & 20 & 10 & 0\end{array}$ 


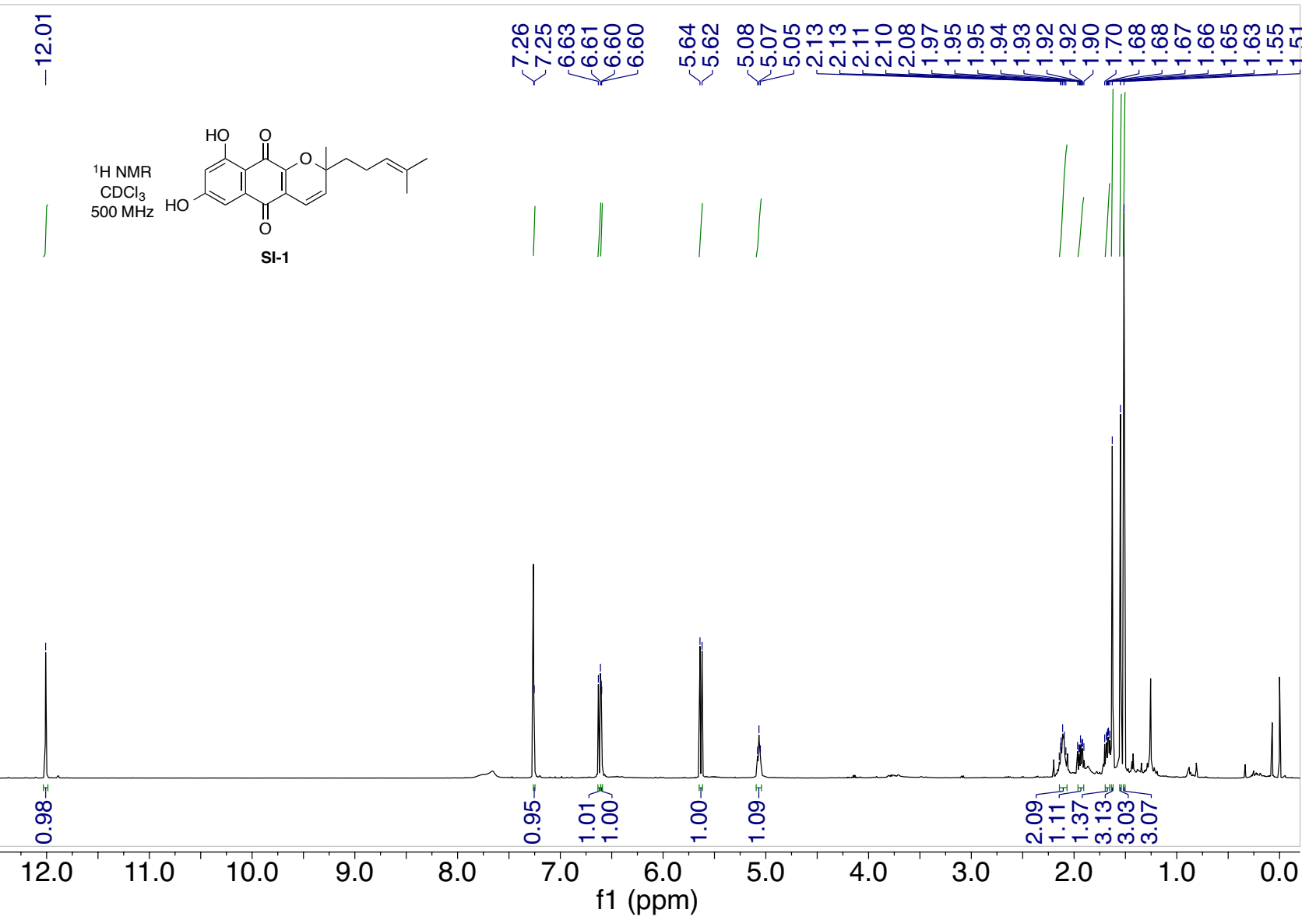




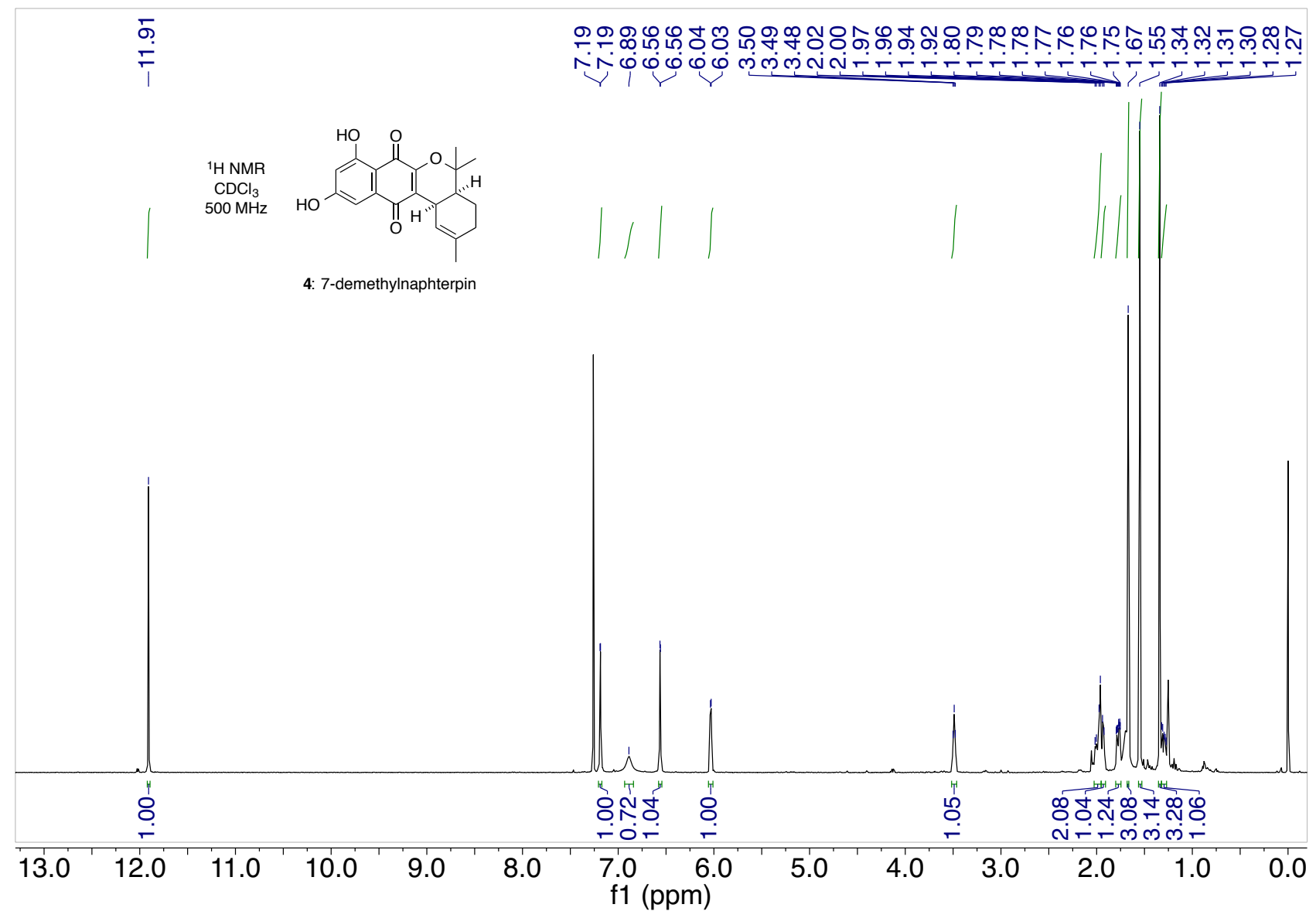

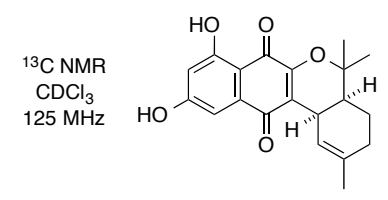

4: 7-demethylnaphterpin

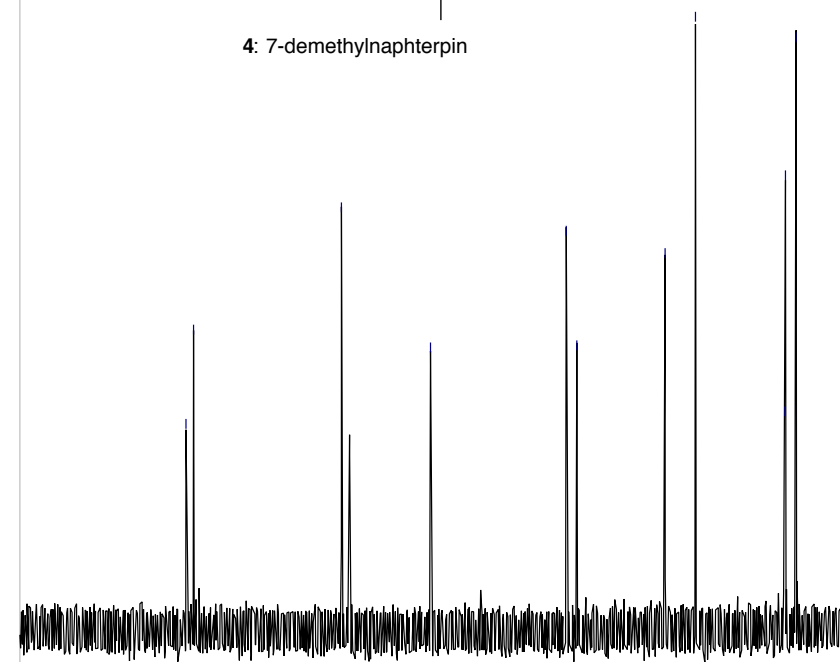

200

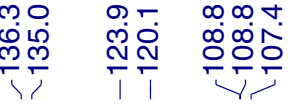

0
$\dot{0}$
0
1

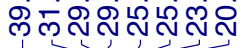

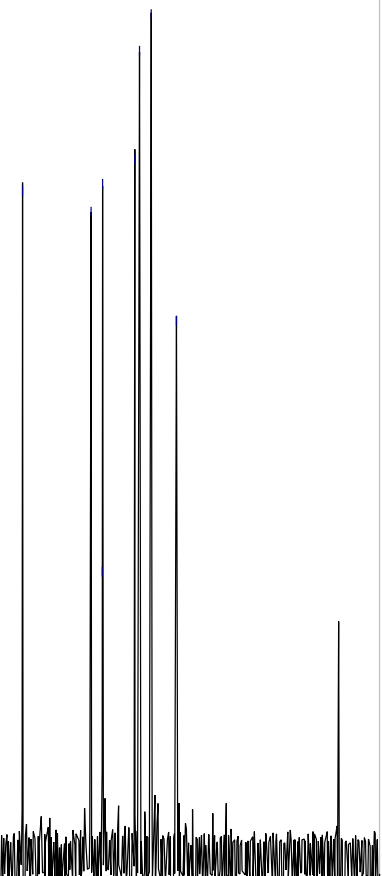

$\mathrm{f} 1$ (ppm) 

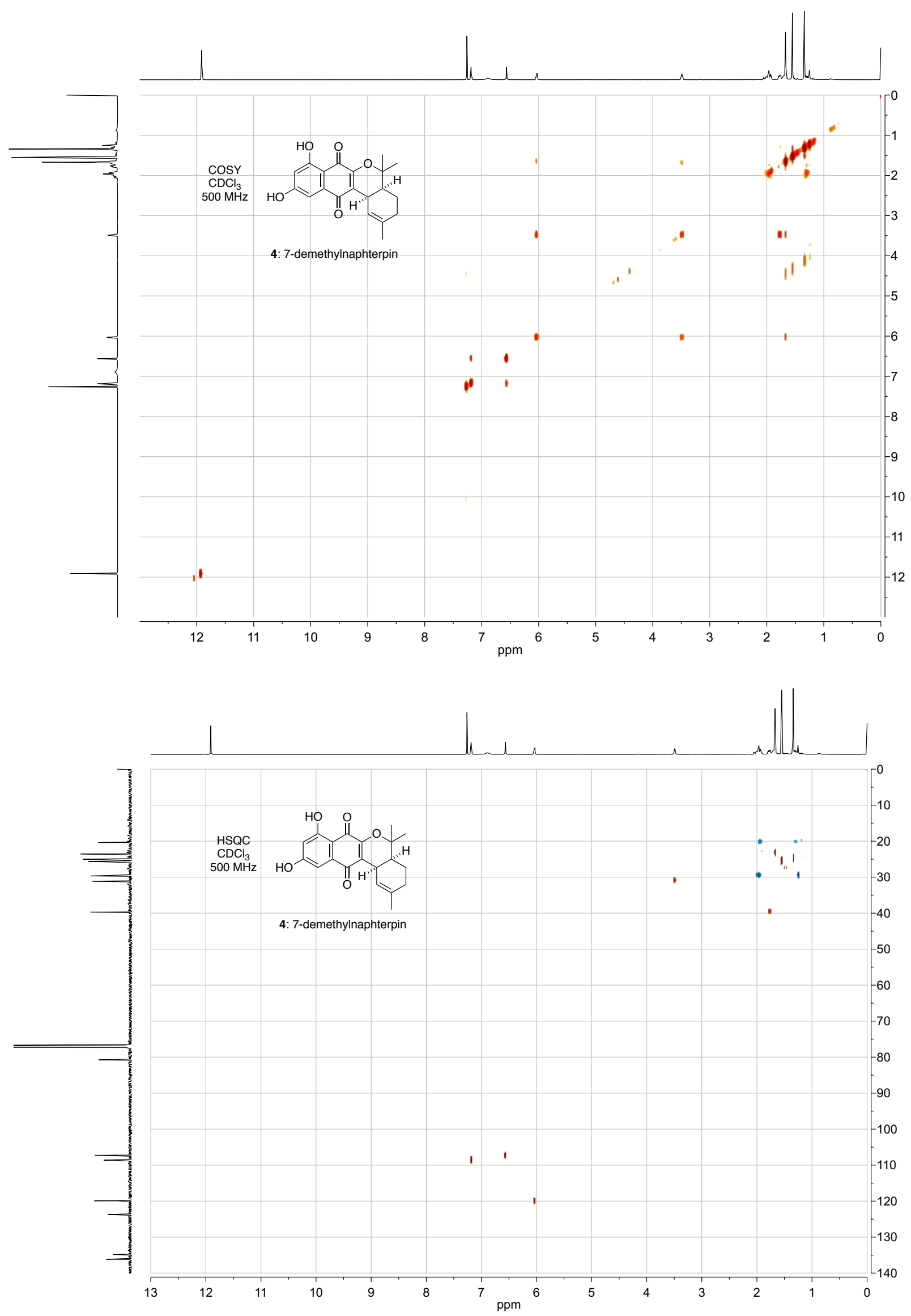


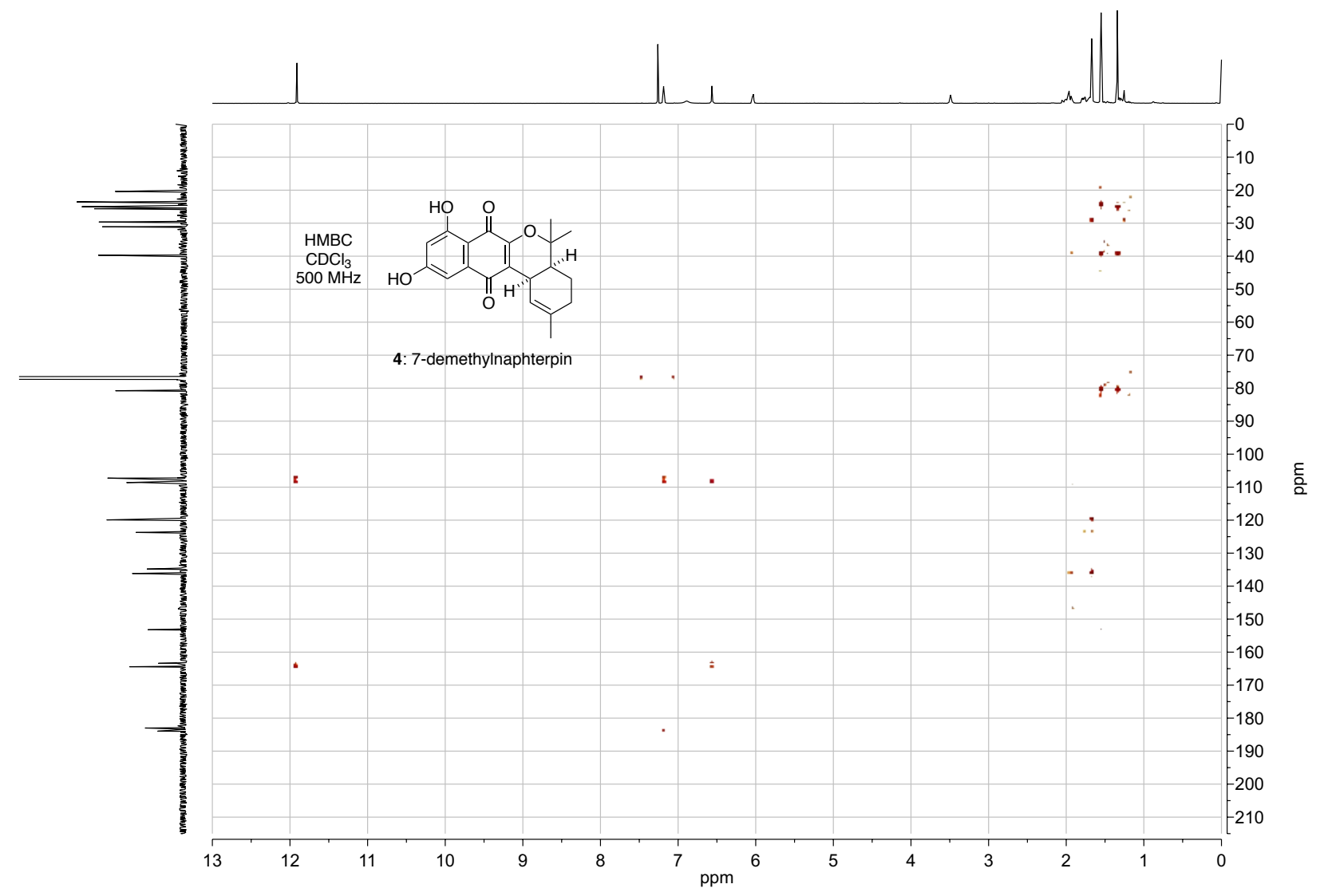



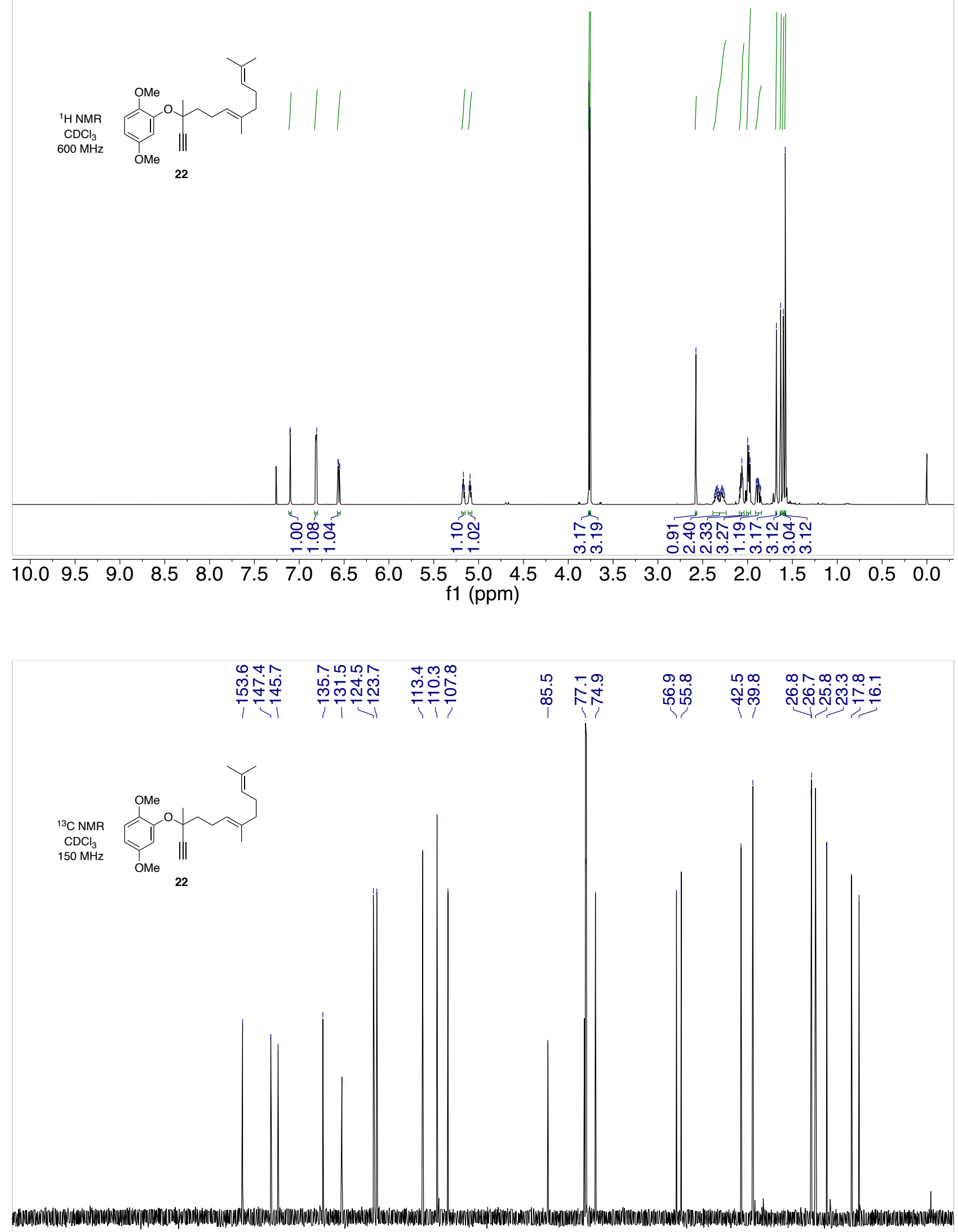

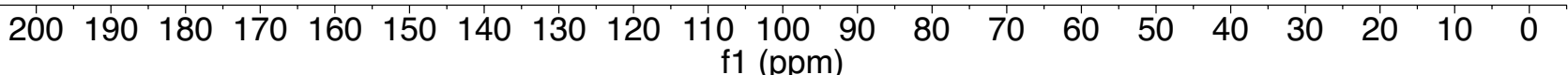




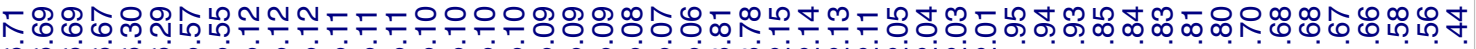
అ
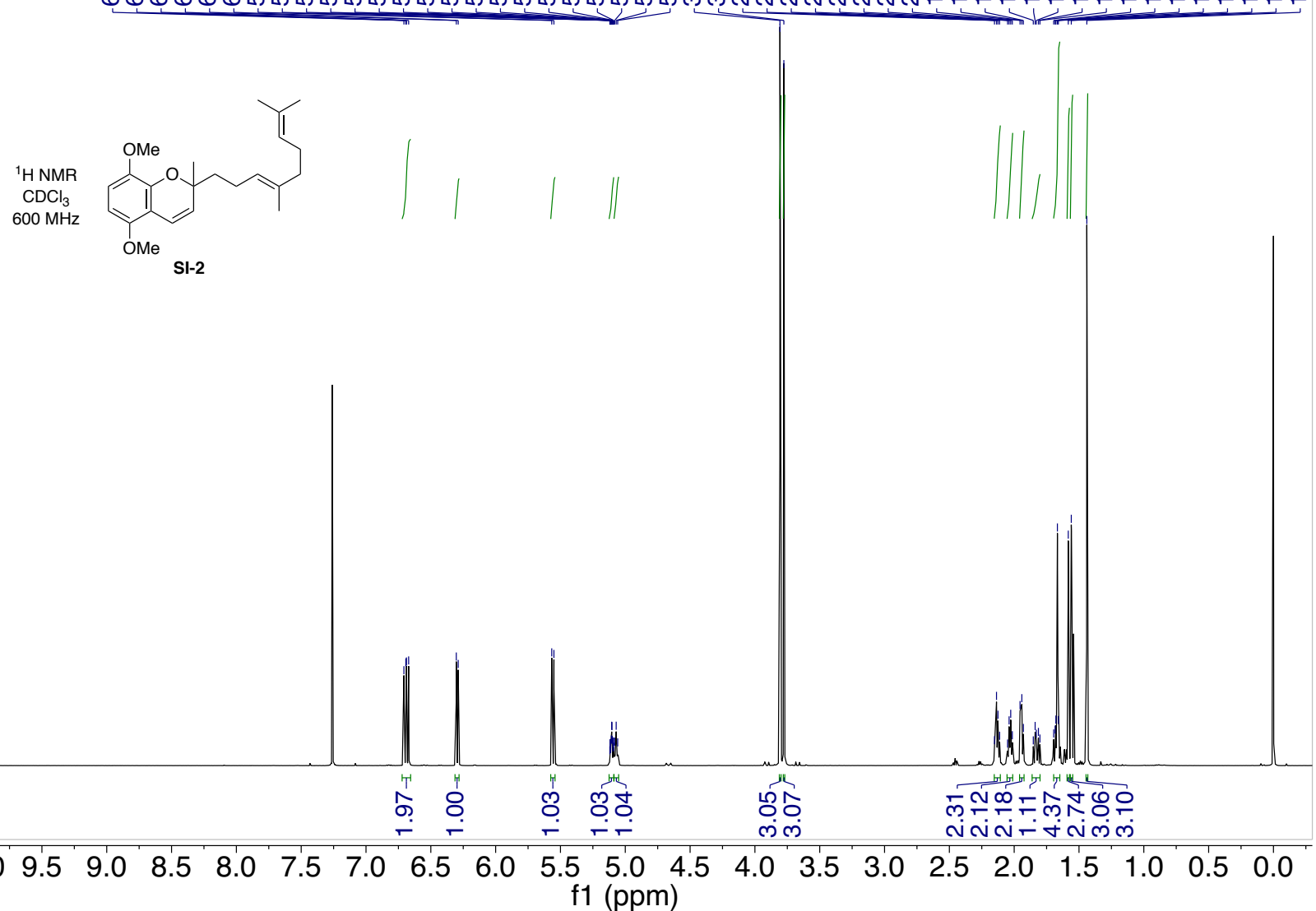

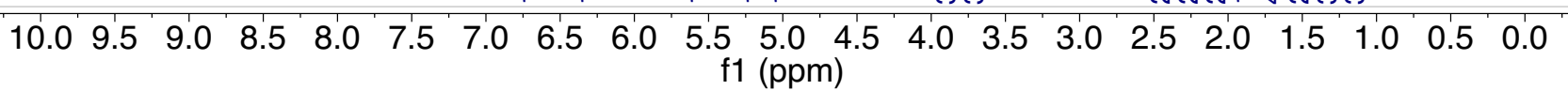

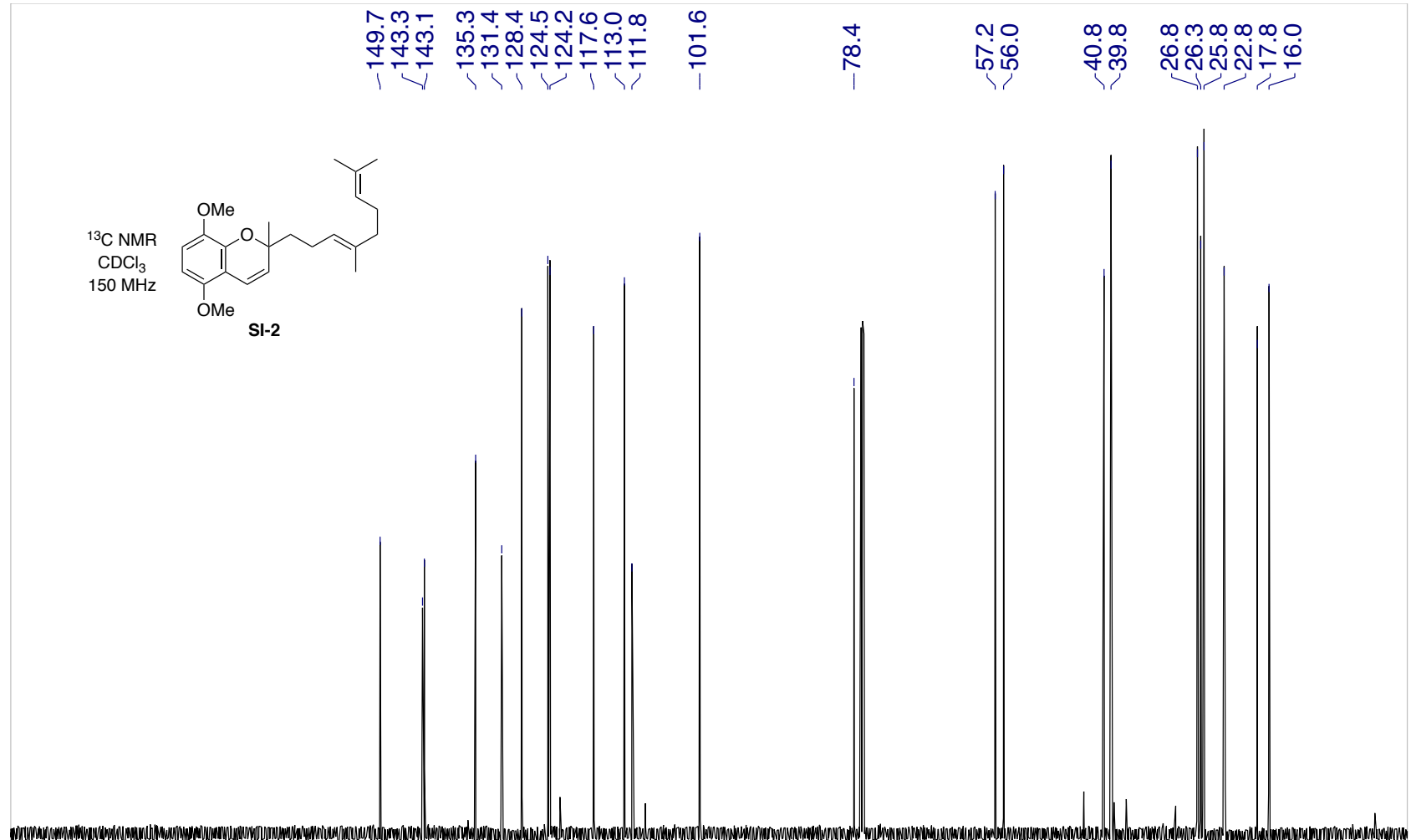

$\begin{array}{llllllllllllllllllllll}200 & 190 & 180 & 170 & 160 & 150 & 140 & 130 & 120 & 110 & 100 & 90 & 80 & 70 & 60 & 50 & 40 & 30 & 20 & 10 & 0\end{array}$ $\mathrm{f1}(\mathrm{ppm})$ 

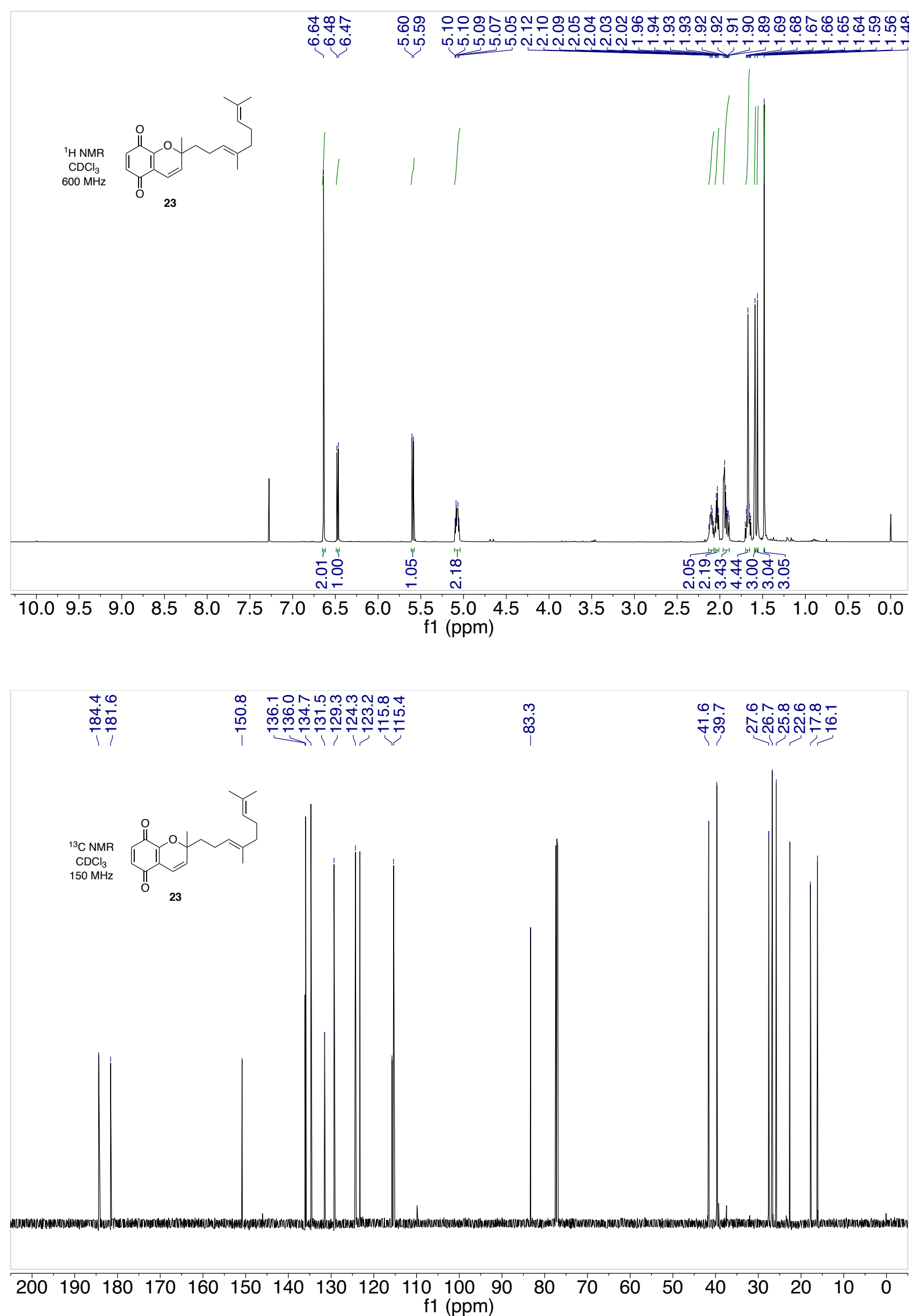


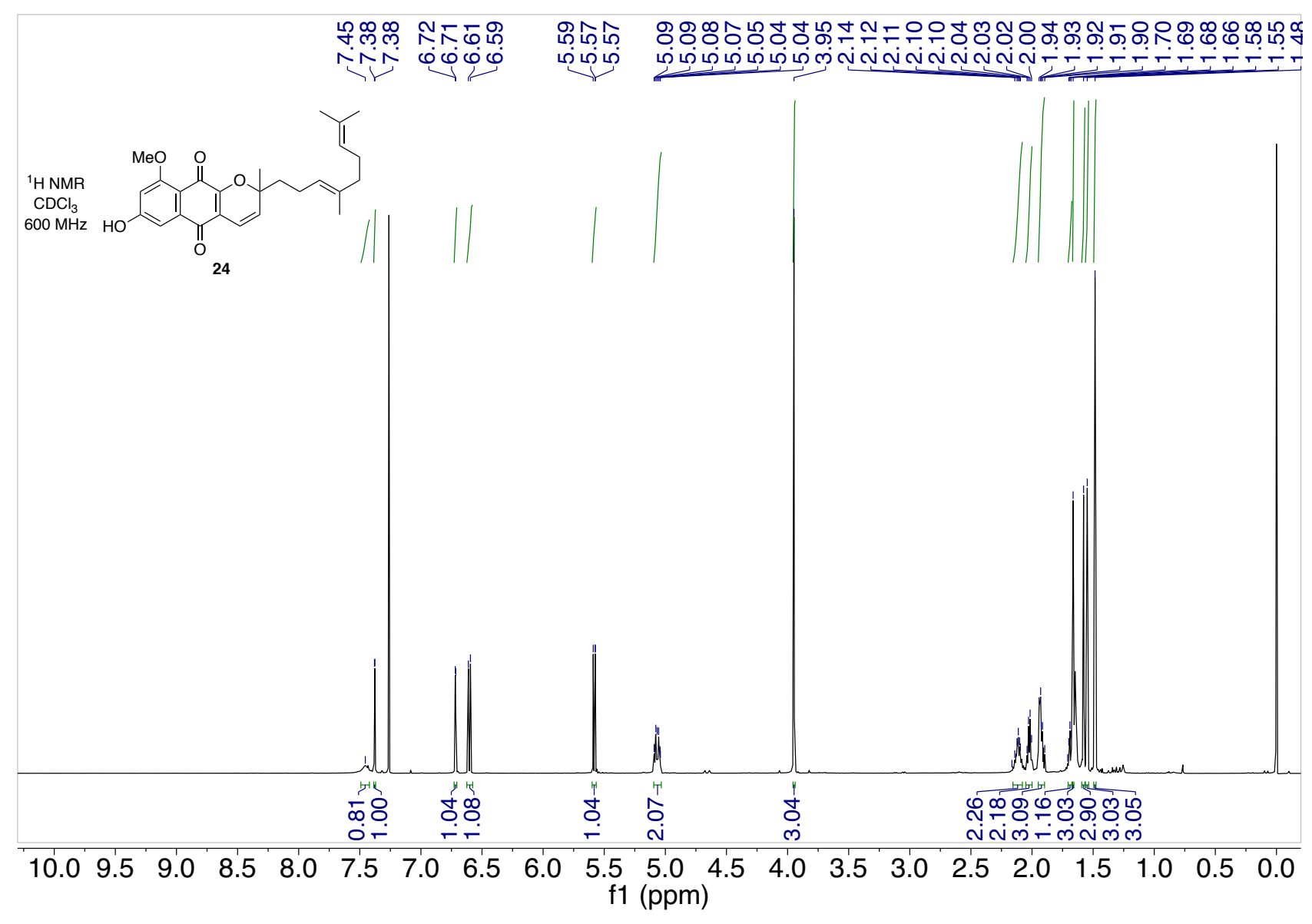

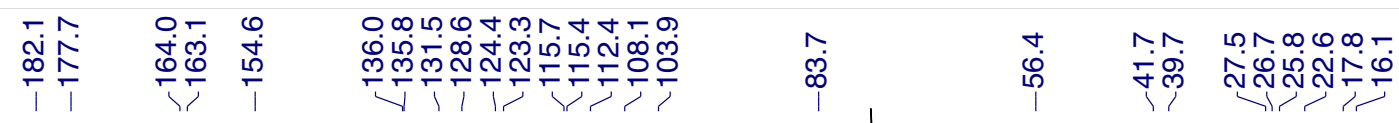
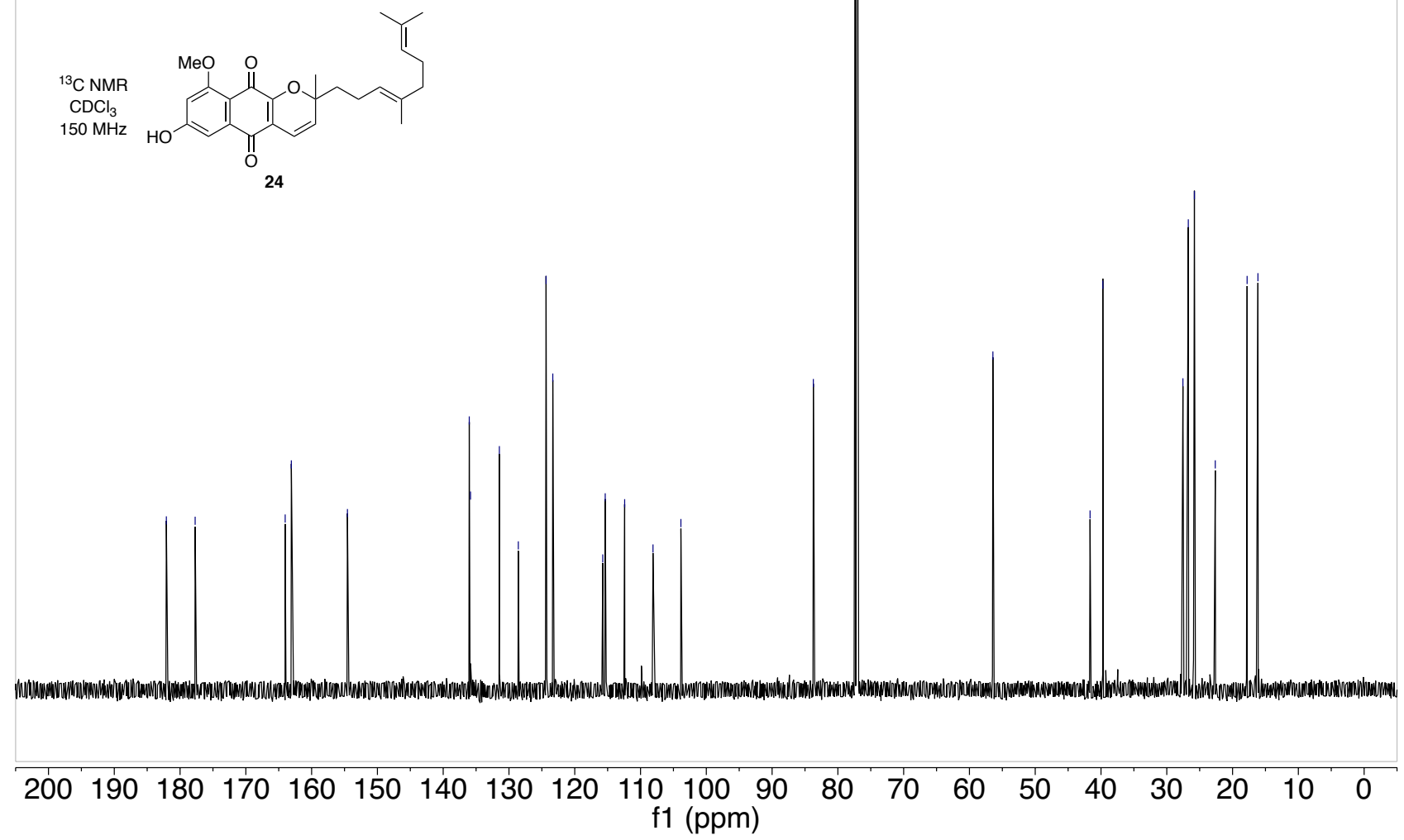

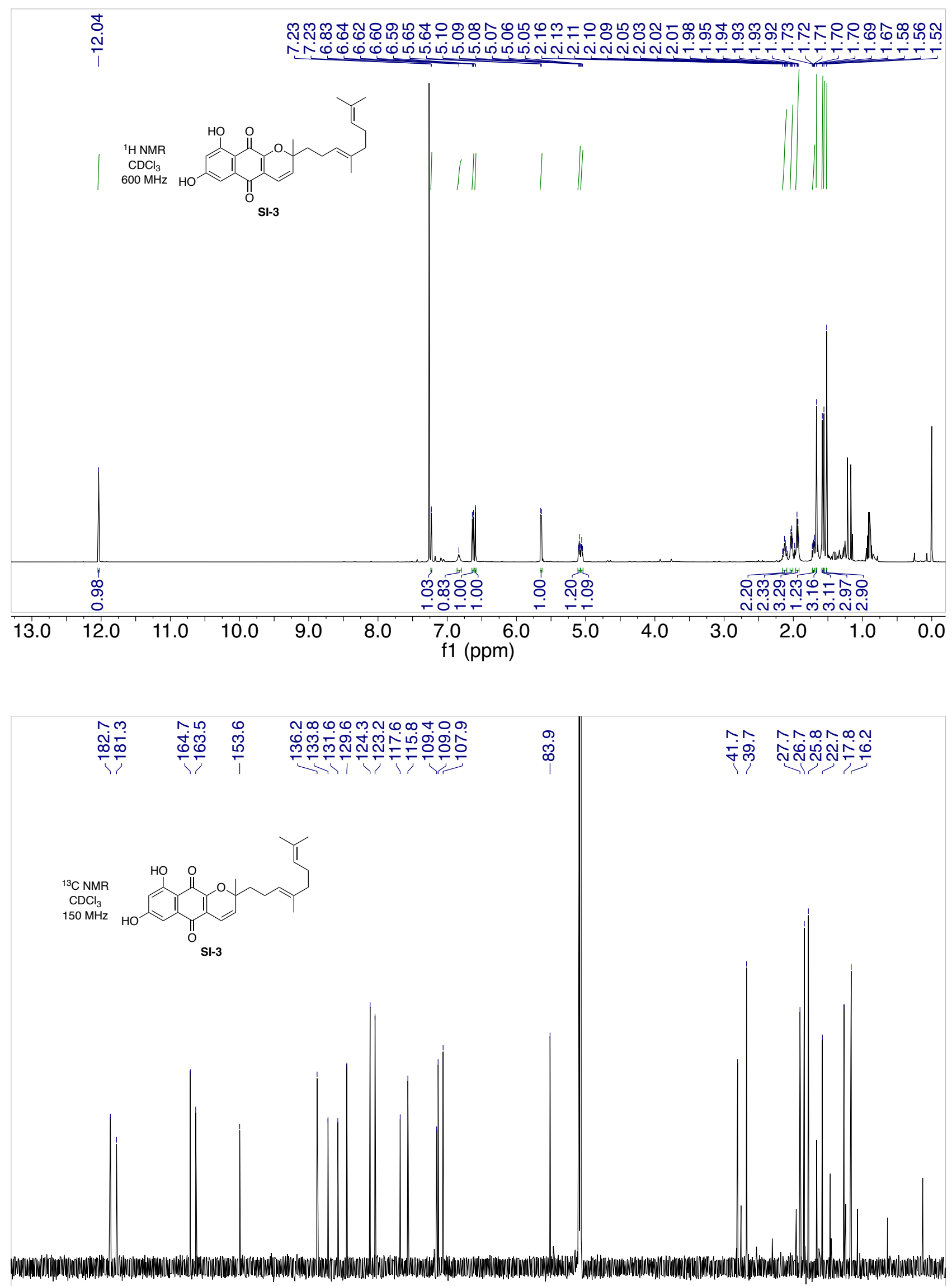

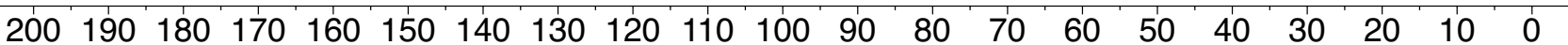
f1 (ppm) 

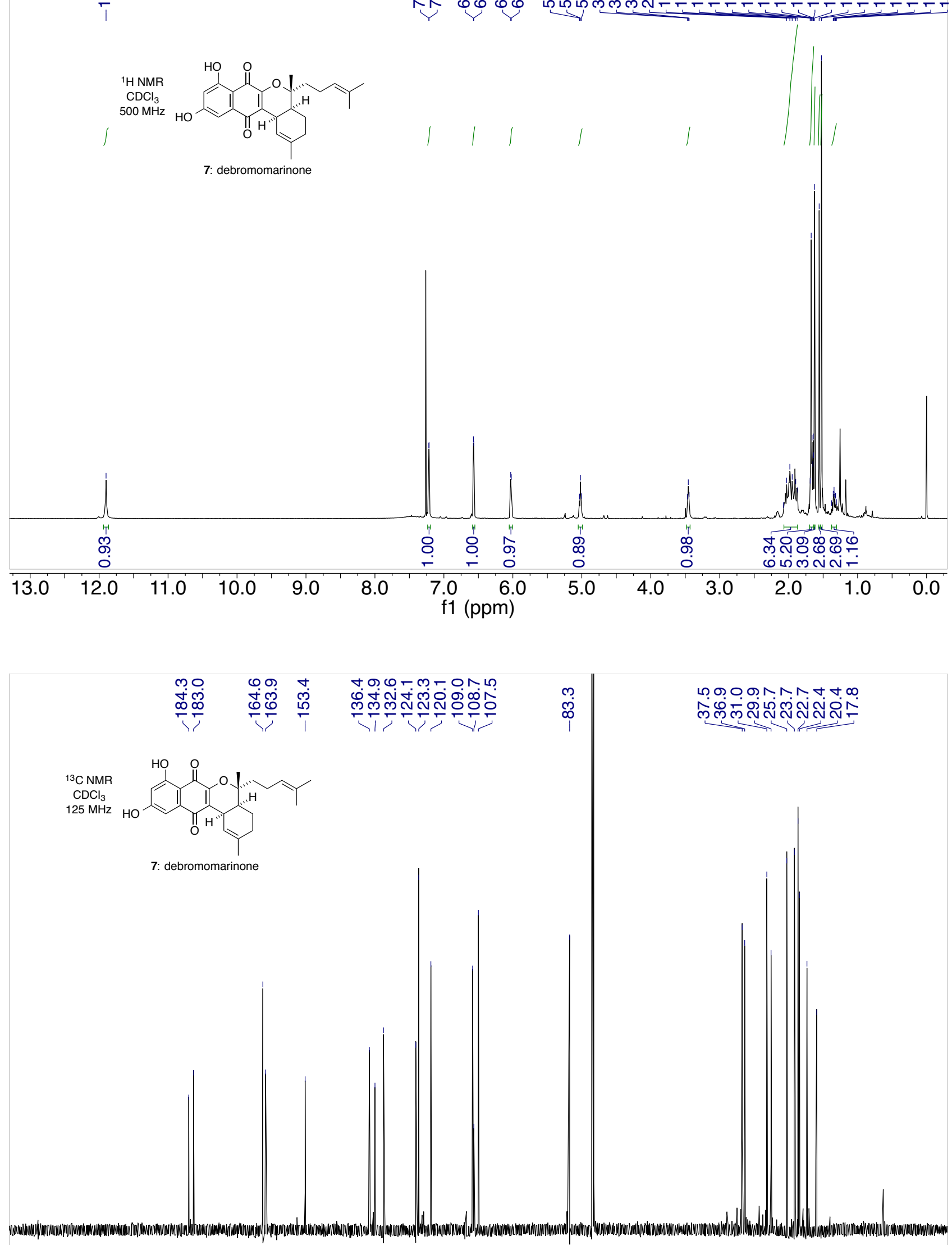

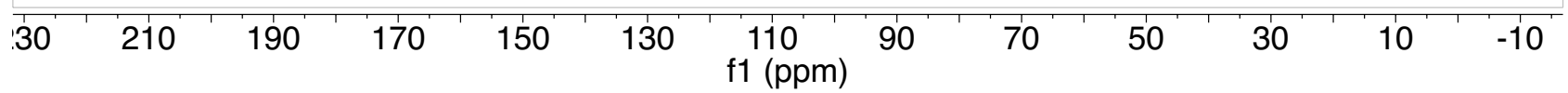



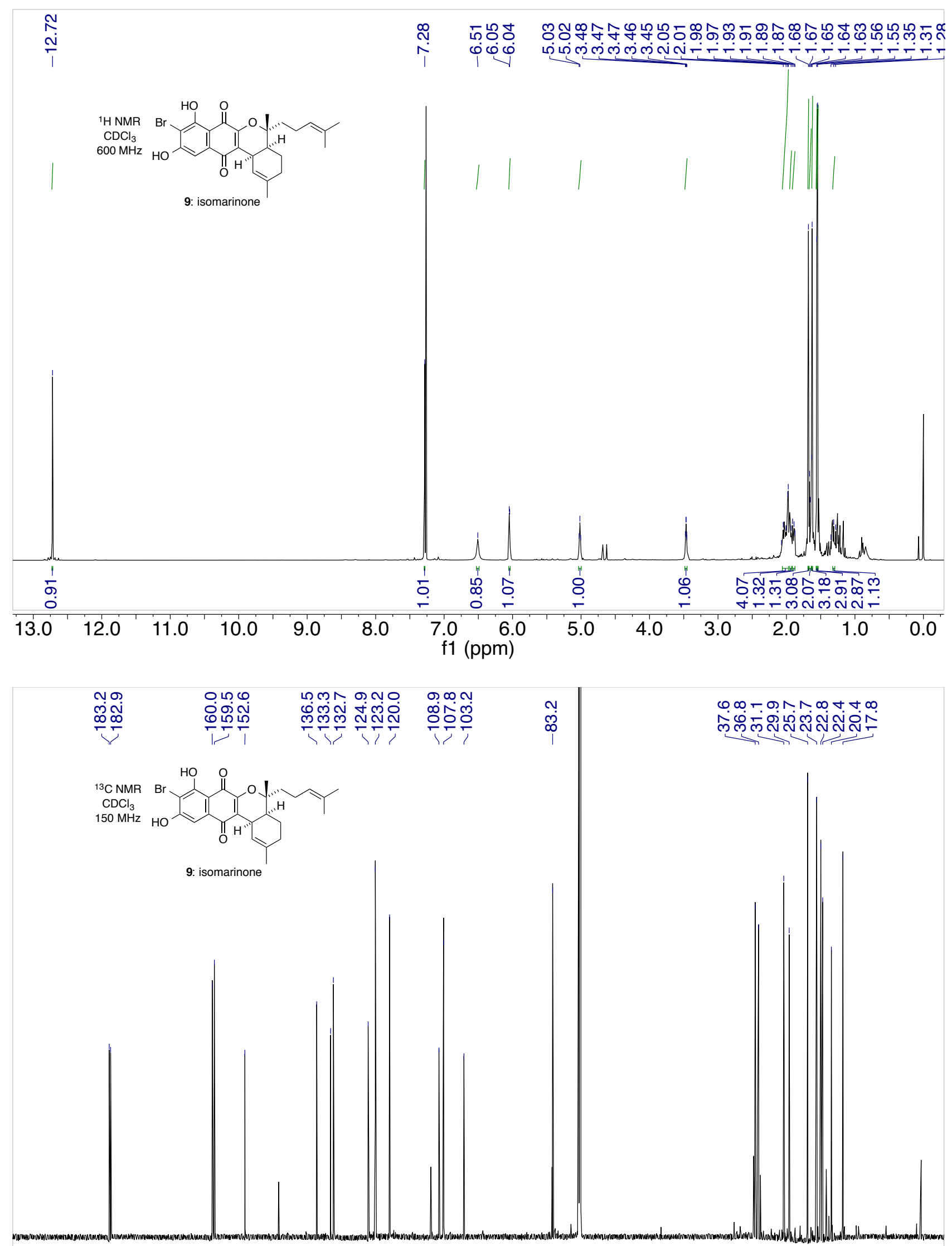

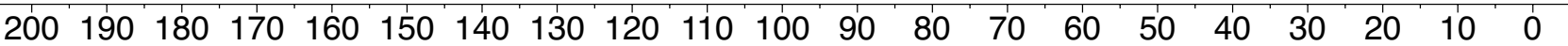
f1 (ppm) 

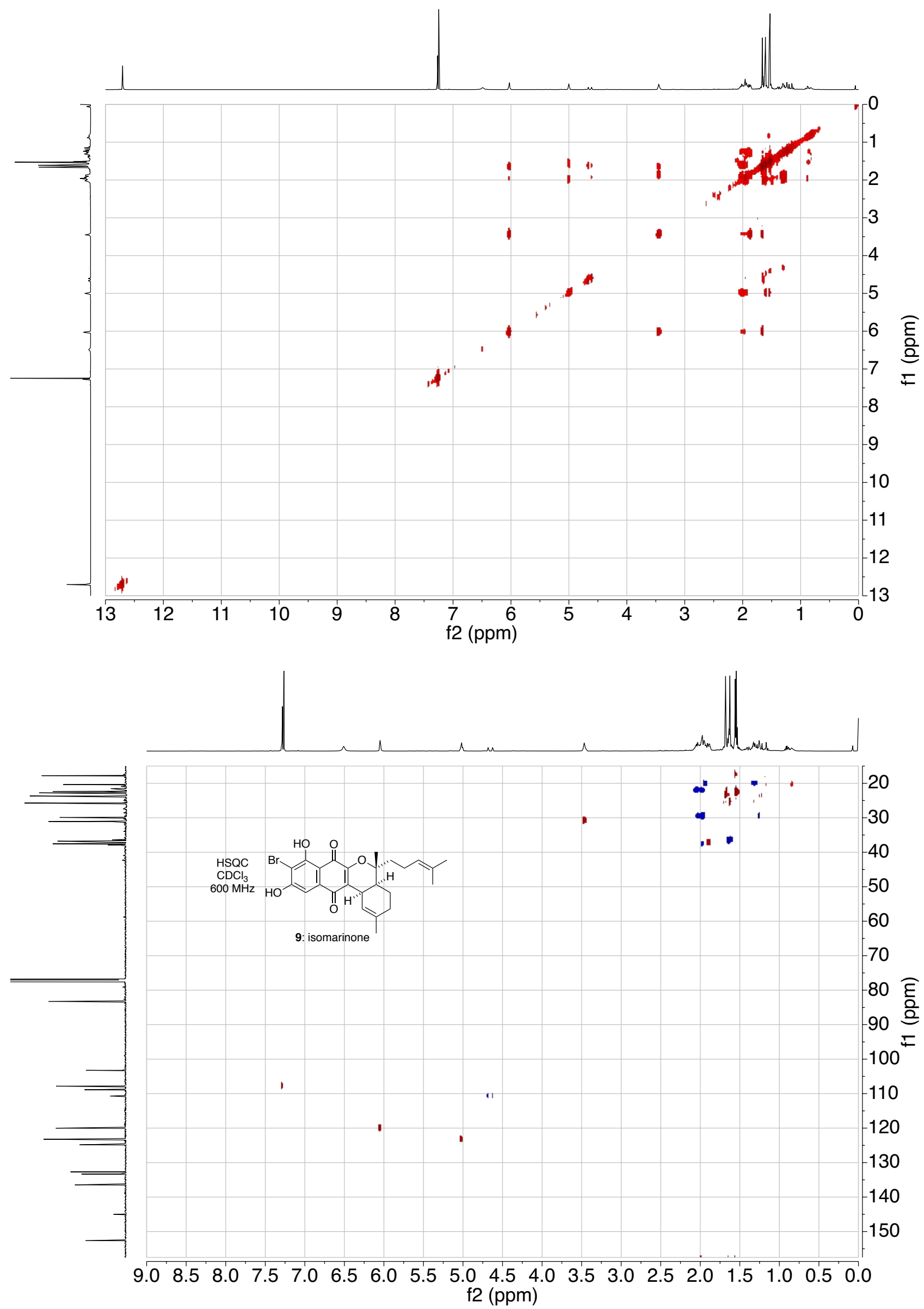


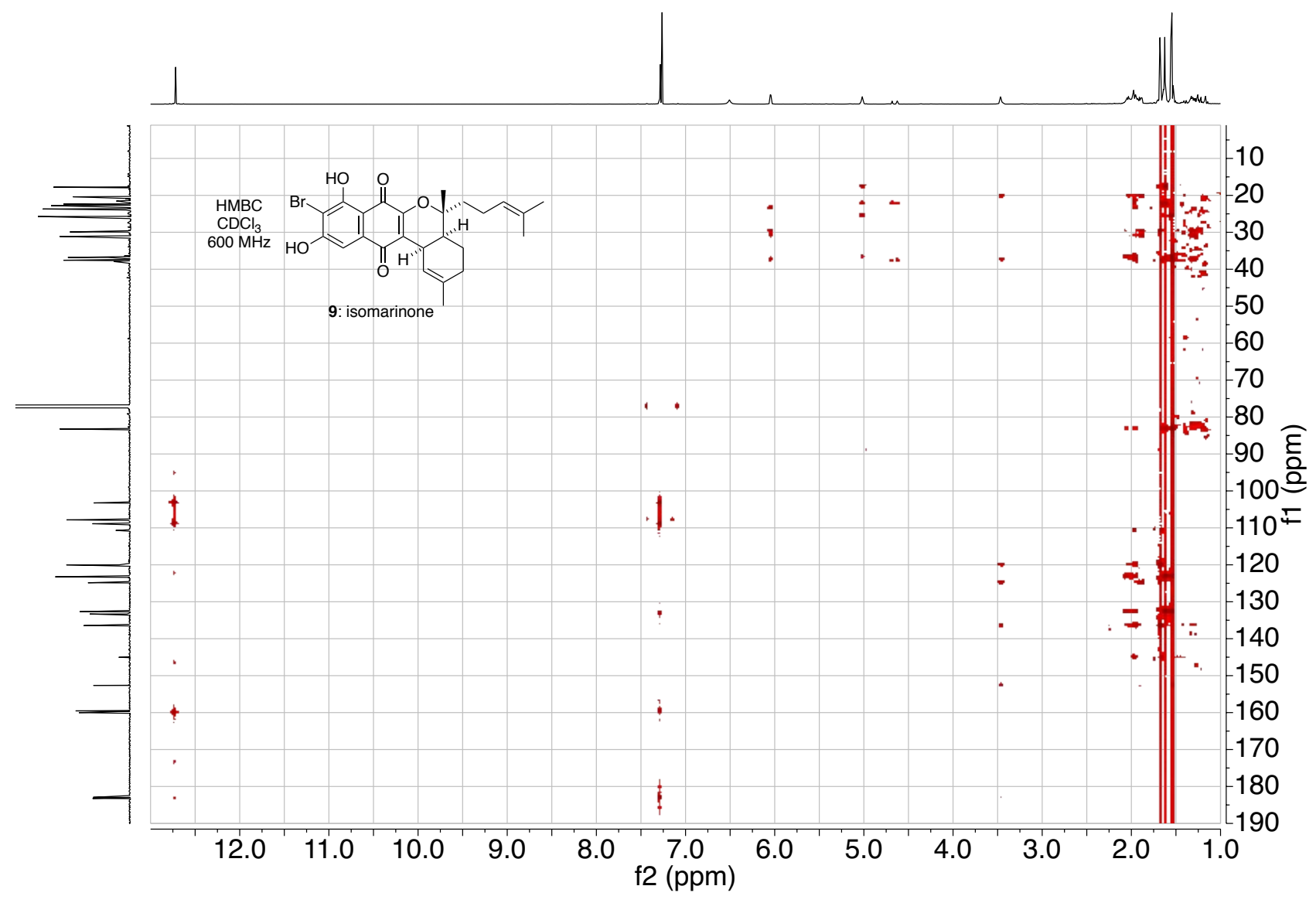



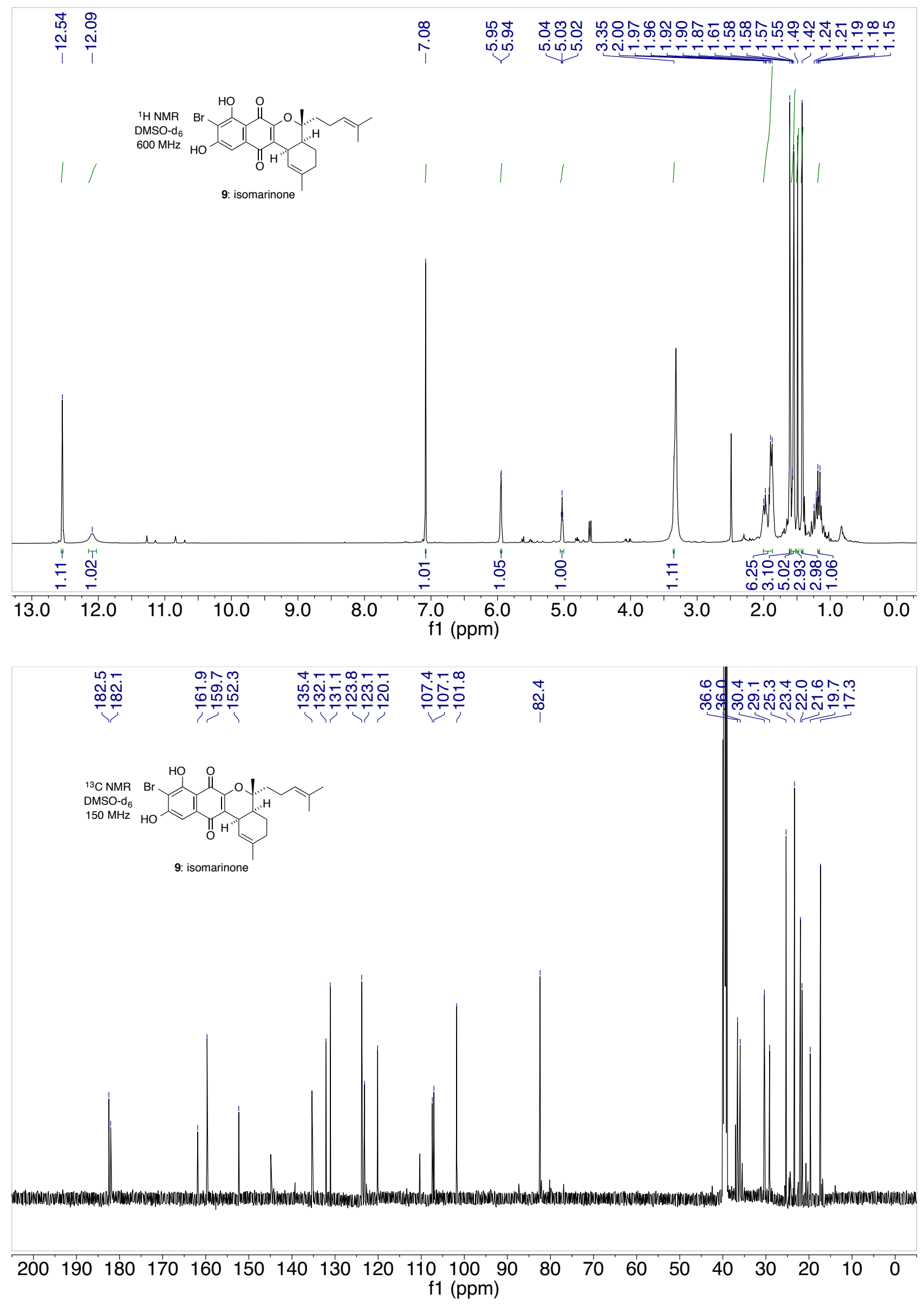

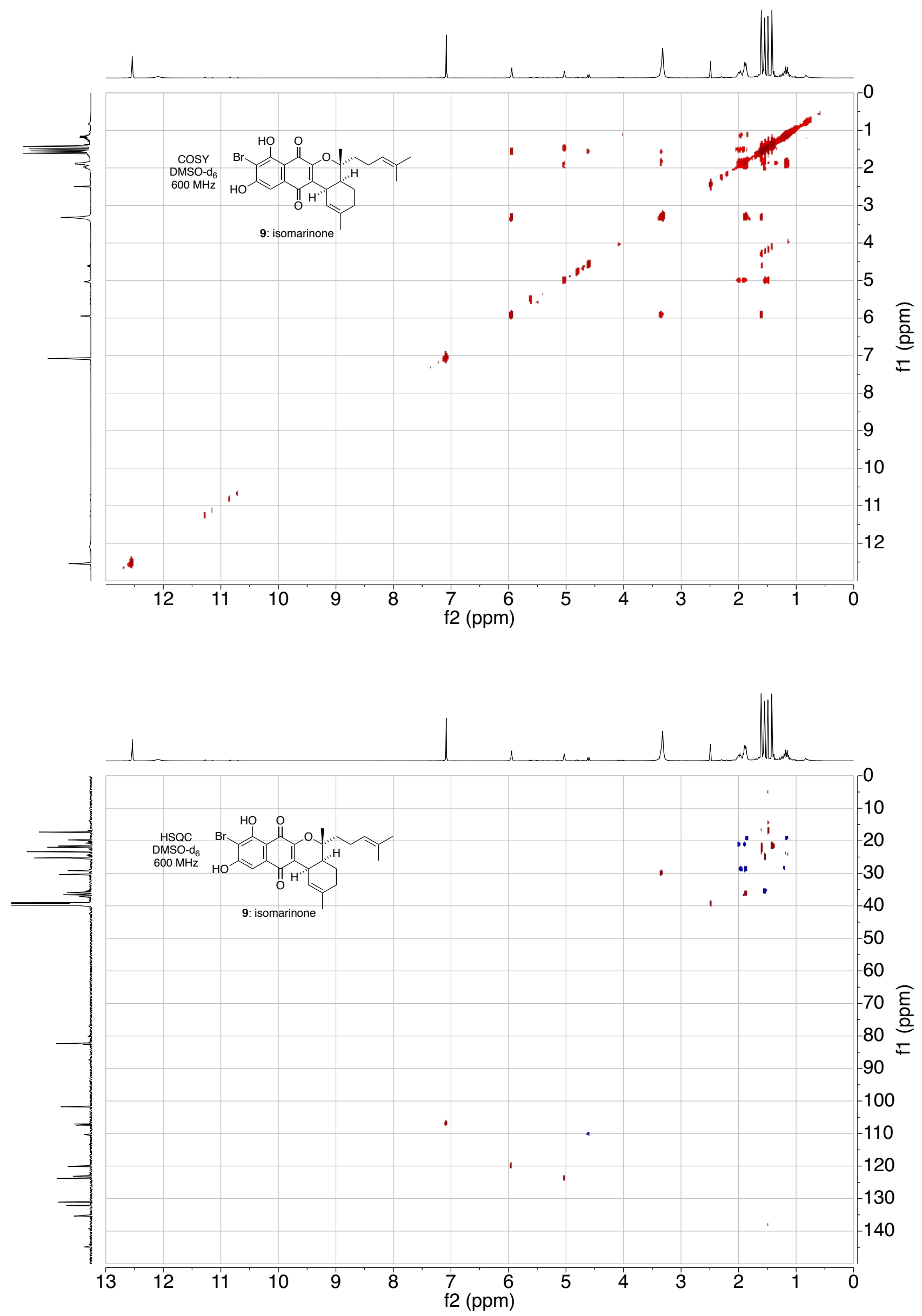

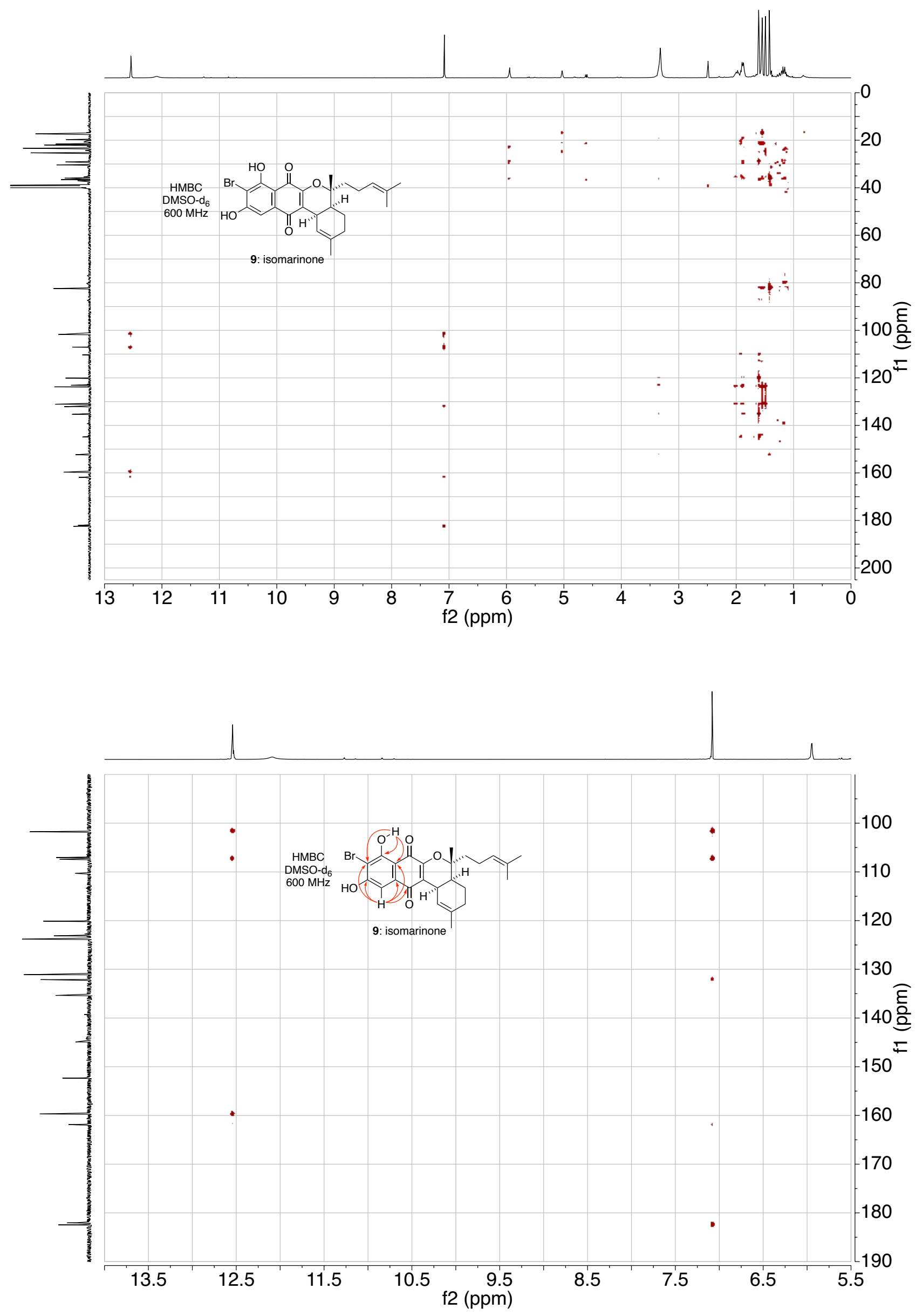


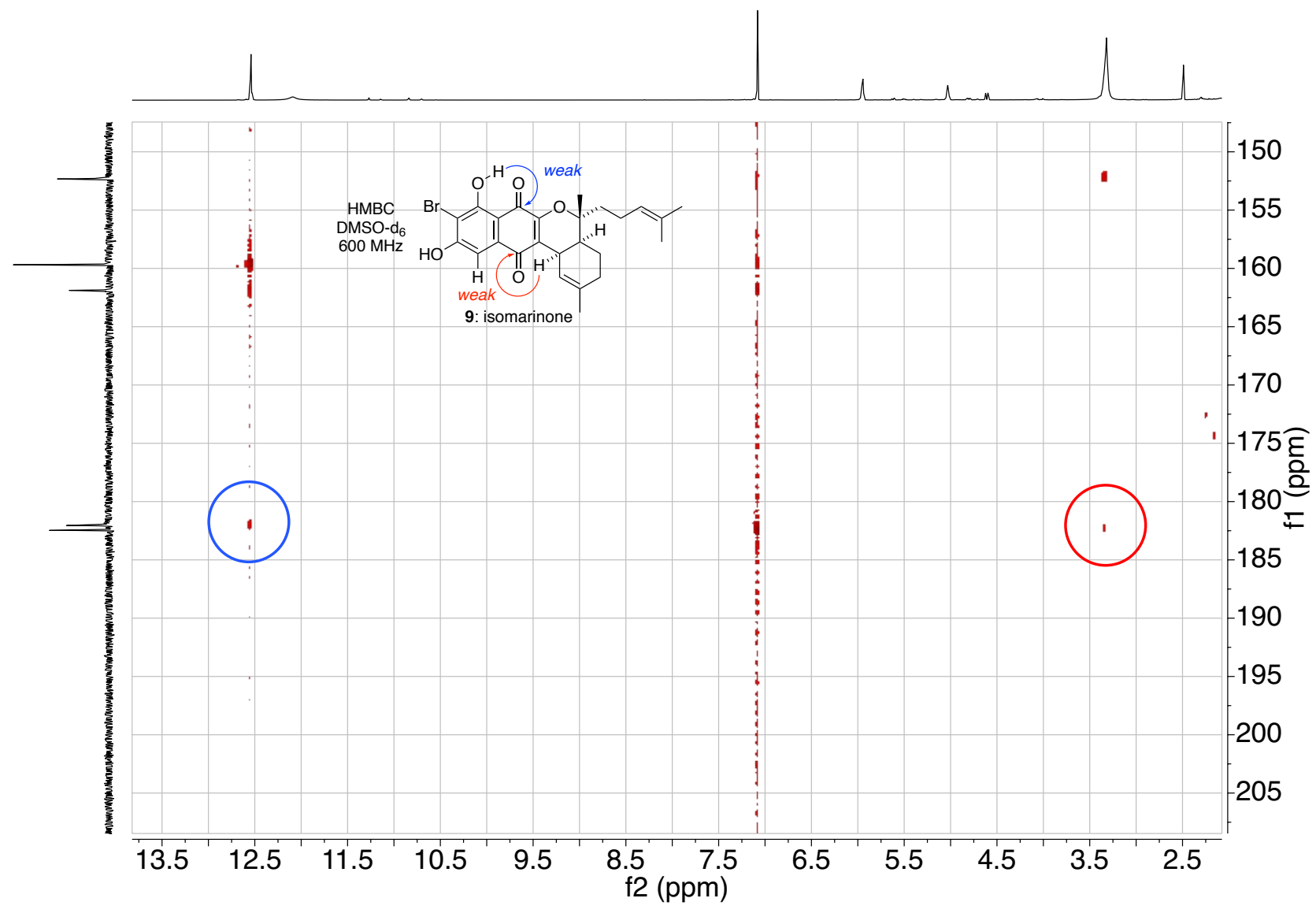




\section{Tables of NMR data}

Table of ${ }^{1} \mathrm{H}$ and ${ }^{13} \mathrm{C}$ NMR data of natural ${ }^{6}$ and synthetic naphterpin (1).

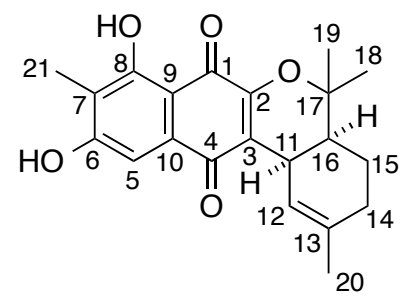

1: naphterpin

\begin{tabular}{|c|c|c|c|c|}
\hline Assignment & $\begin{array}{l}\text { Natural naphterpin, } \\
{ }^{1} \mathrm{H} \text { NMR, } \mathrm{CDCl}_{3}\end{array}$ & $\begin{array}{c}\text { Synthetic naphterpin, } \\
{ }^{1} \mathrm{H} \mathrm{NMR} \mathrm{CDCl}_{3}, \\
500 \mathrm{MHz}\end{array}$ & $\begin{array}{l}\text { Natural naphterpin, } \\
{ }^{13} \mathrm{C} \mathrm{NMR,} \mathrm{CDCl}_{3}\end{array}$ & $\begin{array}{c}\text { Synthetic naphterpin, } \\
{ }^{13} \mathrm{C} \mathrm{NMR}, \mathrm{CDCl}_{3}, \\
125 \mathrm{MHz}\end{array}$ \\
\hline 1 & & & 183.1 & 183.5 \\
\hline 2 & & & 153.5 & 153.2 \\
\hline 3 & & & 123.3 & 123.7 \\
\hline 4 & & & 184.8 & 184.2 \\
\hline 5 & 7.31 & $7.18(\mathrm{~s}, 1 \mathrm{H})$ & 108.4 & 108.5 \\
\hline 6 & & & 161.5 & 160.7 \\
\hline 7 & & & 117.2 & 116.8 \\
\hline 8 & & & 162.6 & 162.5 \\
\hline 9 & & & 107.9 & 107.9 \\
\hline 10 & & & 131.4 & 131.8 \\
\hline 11 & 3.47 & 3.49 (br s) & 31.1 & 31.3 \\
\hline 12 & 6.01 & $6.06(\mathrm{~d}, J=5.2 \mathrm{~Hz}, 1 \mathrm{H})$ & 120.0 & 120.3 \\
\hline 13 & & & 136.1 & 136.2 \\
\hline 14 & 1.95 & $2.08-1.91(\mathrm{~m}, 3 \mathrm{H})$ & 29.6 & 29.8 \\
\hline 15 & $1.25,1.95$ & $\begin{array}{c}2.08-1.91(\mathrm{~m}, 3 \mathrm{H}) \\
1.34-1.27(\mathrm{~m}, 1 \mathrm{H})\end{array}$ & 20.4 & 20.5 \\
\hline 16 & 1.75 & $\begin{array}{c}1.77(\mathrm{ddd}, J=12.1,6.2 \\
2.8 \mathrm{~Hz}, 1 \mathrm{H})\end{array}$ & 39.7 & 39.9 \\
\hline 17 & & & 80.8 & 80.7 \\
\hline 18 & 1.51 & $1.56(\mathrm{~s}, 3 \mathrm{H})$ & 25.6 & 25.8 \\
\hline 19 & 1.34 & $1.34(\mathrm{~s}, 3 \mathrm{H})$ & 25.1 & 25.2 \\
\hline 20 & 1.64 & $1.68(\mathrm{~s}, 3 \mathrm{H})$ & 23.5 & 23.7 \\
\hline 21 & 2.15 & $2.18(\mathrm{~s}, 3 \mathrm{H})$ & 7.8 & 7.9 \\
\hline $6-\mathrm{OH}$ & 8.25 & $6.46(1 \mathrm{H}, \mathrm{s})$ & & \\
\hline $8-\mathrm{OH}$ & 12.20 & $12.29(\mathrm{~s}, 1 \mathrm{H})$ & & \\
\hline
\end{tabular}

${ }^{6}$ Shin-ya, K.; Imai, S.; Furihata, K.; Hayakawa, Y.; Kato, Y.; Vanduyne, G. D.; Clardy, J.; Seto, H. J. Antibiot. 1990, 43, 444. 
Table of ${ }^{1} \mathrm{H}$ and ${ }^{13} \mathrm{C}$ NMR data of natural ${ }^{7}$ and synthetic naphterpin $\mathrm{B}(2)$.

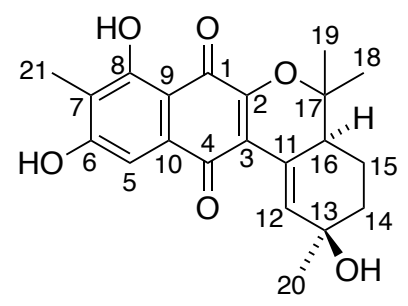

2: naphterpin B

\begin{tabular}{|c|c|c|c|c|}
\hline Assignment & $\begin{array}{c}\text { Natural naphterpin B, } \\
{ }^{1} \mathrm{H} \mathrm{NMR}, \mathrm{CDCl}_{3}\end{array}$ & $\begin{array}{c}\text { Synthetic naphterpin B, } \\
{ }^{1} \mathrm{H} \mathrm{NMR}, \mathrm{CDCl}_{3}, \\
600 \mathrm{MHz}\end{array}$ & $\begin{array}{c}\text { Natural naphterpin B, } \\
{ }^{13} \mathrm{C} \text { NMR, } \mathrm{CDCl}_{3}\end{array}$ & $\begin{array}{c}\text { Synthetic naphterpin B, } \\
{ }^{13} \mathrm{C} \mathrm{NMR,} \mathrm{CDCl}_{3}, \\
150 \mathrm{MHz}\end{array}$ \\
\hline 1 & & & 182.4 & 182.5 \\
\hline 2 & & & 154.5 & 154.5 \\
\hline 3 & & & 127.0 & 127.1 \\
\hline 4 & & & 183.3 & 183.4 \\
\hline 5 & 7.16 & 7.20 & 108.5 & 108.5 \\
\hline 6 & & & 161.8 & 161.6 \\
\hline 7 & & & 117.0 & 117.0 \\
\hline 8 & & & 162.2 & 162.3 \\
\hline 9 & & & 107.9 & 108.0 \\
\hline 10 & & & 131.4 & 131.6 \\
\hline 11 & & & 116.1 & 116.2 \\
\hline 12 & 7.46 & 7.46 & 135.3 & 135.5 \\
\hline 13 & & & 68.5 & 68.4 \\
\hline 14 & $1.61,1.95$ & $\begin{array}{l}1.64-1.58(\mathrm{~m}), \\
1.98-1.92(\mathrm{~m})\end{array}$ & 35.7 & 35.8 \\
\hline 15 & $1.61,1.80$ & $\begin{array}{l}1.64-1.58(\mathrm{~m}) \\
1.83-1.79(\mathrm{~m})\end{array}$ & 20.1 & 20.2 \\
\hline 16 & 2.30 & $2.35-2.28(\mathrm{~m})$ & 42.4 & 42.4 \\
\hline 17 & & & 82.4 & 82.4 \\
\hline 18 & 1.12 & $1.16(\mathrm{~s})$ & 19.3 & 19.5 \\
\hline 19 & 1.56 & $1.57(\mathrm{~s})$ & 26.7 & 26.7 \\
\hline 20 & 1.52 & $1.49(\mathrm{~s})$ & 30.7 & 30.7 \\
\hline 21 & 2.12 & $2.12(\mathrm{~s})$ & 7.7 & 7.8 \\
\hline $6-\mathrm{OH}$ & N. D. & $8.17(\mathrm{~s})$ & & \\
\hline $8-\mathrm{OH}$ & 12.2 & $12.24(\mathrm{~s})$ & & \\
\hline
\end{tabular}

${ }^{7}$ Takagi, H.; Motohashi, K.; Miyamoto, T.; Shin-ya, K.; Furihata, K.; Seto, H. J. Antibiot. 2005, 58, 275. 
Table of ${ }^{1} \mathrm{H}$ and ${ }^{13} \mathrm{C}$ NMR data of natural ${ }^{8}$ and synthetic isomarinone (9).

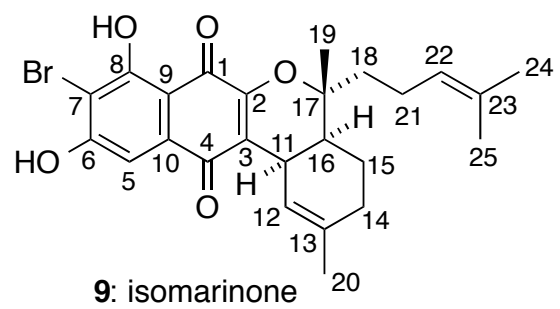

\begin{tabular}{|c|c|c|c|c|}
\hline Assignment & $\begin{array}{c}\text { Natural isomarinone, } \\
{ }^{1} \mathrm{H} \text { NMR, DMSO-d6 } \\
300 \mathrm{MHz}\end{array}$ & $\begin{array}{c}\text { Synthetic isomarinone, } \\
{ }^{1} \mathrm{H} \text { NMR, DMSO-d6 } \\
600 \mathrm{MHz}\end{array}$ & $\begin{array}{c}\text { Natural isomarinone, } \\
{ }^{13} \mathrm{C} \text { NMR, DMSO-d6 } \\
300 \mathrm{MHz}\end{array}$ & $\begin{array}{c}\text { Synthetic isomarinone, } \\
{ }^{13} \mathrm{C} \text { NMR, DMSO-d6 } \\
600 \mathrm{MHz}\end{array}$ \\
\hline 1 & & & 182.0 & 182.1 \\
\hline 2 & & & 152.3 & 152.3 \\
\hline 3 & & & 123.1 & 123.1 \\
\hline 4 & & & 182.4 & 182.5 \\
\hline 5 & $7.09(\mathrm{~s}, 1 \mathrm{H})$ & $7.08(\mathrm{~s}, 1 \mathrm{H})$ & 107.4 & 107.4 \\
\hline 6 & & & 161.8 & 161.9 \\
\hline 7 & & & 101.7 & 101.8 \\
\hline 8 & & & 159.6 & 159.7 \\
\hline 9 & & & 107.0 & 107.1 \\
\hline 10 & & & 132.1 & 132.1 \\
\hline 11 & $3.35(\mathrm{~m}, 1 \mathrm{H})$ & $3.36-3.34(\mathrm{~m}, 1 \mathrm{H})$ & 30.3 & 30.4 \\
\hline 12 & $5.95(\mathrm{~d}, J=5.0 \mathrm{~Hz}, 1 \mathrm{H})$ & $5.95(\mathrm{~d}, J=5.0 \mathrm{~Hz}, 1 \mathrm{H})$ & 120.1 & 120.1 \\
\hline 13 & & & 135.3 & 135.4 \\
\hline 14 & $1.84(\mathrm{~m}, 2 \mathrm{H})$ & $2.04-1.84(\mathrm{~m}, 2 \mathrm{H})$ & 29.1 & 29.2 \\
\hline 15 & $\begin{array}{l}1.87(\mathrm{~m}, 1 \mathrm{H}), \\
1.18(\mathrm{~m}, 1 \mathrm{H})\end{array}$ & $\begin{array}{l}1.93-1.84(\mathrm{~m} 1 \mathrm{H}) \\
1.22-1.14(\mathrm{~m} 1 \mathrm{H})\end{array}$ & 19.7 & 19.7 \\
\hline 16 & $1.86(\mathrm{~m}, 1 \mathrm{H})$ & $1.93-1.84(\mathrm{~m}, 1 \mathrm{H})$ & 36.6 & 36.6 \\
\hline 17 & & & 82.4 & 82.4 \\
\hline 18 & $1.54(\mathrm{~m}, 2 \mathrm{H})$ & $1.59-1.54(\mathrm{~m}, 2 \mathrm{H})$ & 35.9 & 36.0 \\
\hline 19 & $1.43(\mathrm{~s}, 3 \mathrm{H})$ & $1.42(\mathrm{~s}, 3 \mathrm{H})$ & 21.6 & 21.6 \\
\hline 20 & $1.61(\mathrm{~s}, 3 \mathrm{H})$ & $1.61(\mathrm{~s}, 3 \mathrm{H})$ & 23.3 & 23.4 \\
\hline 21 & $1.94(\mathrm{~m}, 2 \mathrm{H})$ & $2.04-1.84(\mathrm{~m}, 2 \mathrm{H})$ & 22.0 & 22.0 \\
\hline 22 & $5.04(\mathrm{bt}, J=6.8 \mathrm{~Hz}, 1 \mathrm{H})$ & $5.03(\mathrm{bt}, J=6.5 \mathrm{~Hz}, 1 \mathrm{H})$ & 123.8 & 123.8 \\
\hline 23 & & & 131.1 & 131.1 \\
\hline 24 & $1.55(\mathrm{~s}, 3 \mathrm{H})$ & $1.55(\mathrm{~s}, 3 \mathrm{H})$ & 25.3 & 25.3 \\
\hline 25 & $1.50(\mathrm{~s}, 3 \mathrm{H})$ & $1.49(\mathrm{~s}, 3 \mathrm{H})$ & 17.3 & 17.3 \\
\hline $6-\mathrm{OH}$ & $12.11(\mathrm{~s}, 1 \mathrm{H})$ & $12.09(\mathrm{~s}, 1 \mathrm{H})$ & & \\
\hline $8-\mathrm{OH}$ & $12.54(\mathrm{~s}, 1 \mathrm{H})$ & $12.54(\mathrm{~s}, 1 \mathrm{H})$ & & \\
\hline
\end{tabular}

${ }^{8}$ Hardt, I. H.; Jensen, P. R.; Fenical, W. Tetrahedron Lett. 2000, 41, 2073. 
Table of ${ }^{1} \mathrm{H}$ and ${ }^{13} \mathrm{C}$ NMR data of natural naphthgeranine $\mathrm{C}^{9}(\mathbf{6})$ and naphthoquinone $\mathrm{C}^{10}(\mathbf{2 5})$.
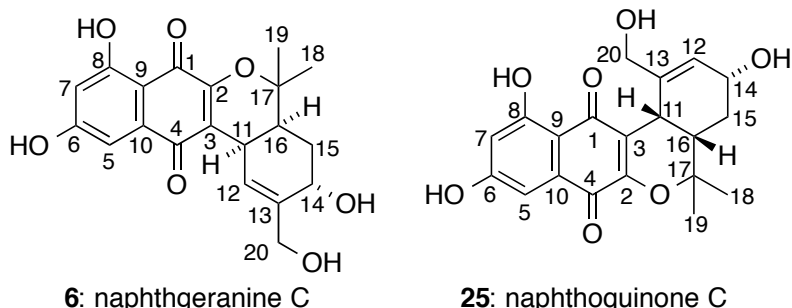

6: naphthgeranine $\mathrm{C}$

25: naphthoquinone $\mathrm{C}$

\begin{tabular}{|c|c|c|c|c|c|c|}
\hline Assignment & $\begin{array}{c}\text { Naphthgeranine } \mathrm{C}, \\
{ }^{1} \mathrm{H} \mathrm{NMR}, \mathrm{CD}_{3} \mathrm{OD}, \\
200 \mathrm{MHz}\end{array}$ & $\begin{array}{c}\text { Naphthoquinone C, } \\
{ }^{1} \mathrm{H} \text { NMR, } \mathrm{CD}_{3} \mathrm{OD}, \\
500 \mathrm{MHz}\end{array}$ & $\begin{array}{l}\text { Naphthgeranine } \mathrm{C}, \\
{ }^{13} \mathrm{C} \mathrm{NMR}, \mathrm{CDCl}_{3-} \\
\mathrm{CD}_{3} \mathrm{OD}, 125 \mathrm{MHz}\end{array}$ & $\begin{array}{c}\text { Naphthoquinone } \mathrm{C}, \\
{ }^{13} \mathrm{C} \mathrm{NMR,} \mathrm{CD}_{3} \mathrm{OD}, \\
125 \mathrm{MHz}\end{array}$ & $\begin{array}{c}\text { Naphthgeranine } \mathrm{C}, \\
\text { Calculated }{ }^{13} \mathrm{C} \text { NMR } \\
\text { SMD: } \mathrm{CD}_{3} \mathrm{OD},\end{array}$ & $\begin{array}{l}\text { Naphthoquinone } \mathrm{C}, \\
\text { Calculated }{ }^{13} \mathrm{C} \text { NMR } \\
\text { SMD: } \mathrm{CD}_{3} \mathrm{OD}\end{array}$ \\
\hline 1 & & & 182.3 & 183.1 & 181.9 & 188.7 \\
\hline 2 & & & 154.1 & 155.0 & 156.0 & 155.1 \\
\hline 3 & & & 121.3 & 122.8 & 122.8 & 125.0 \\
\hline 4 & & & 184.1 & 184.3 & 184.0 & 180.1 \\
\hline 5 & $6.95(\mathrm{~d}, J=2)$ & $6.94(\mathrm{~d}, J=1.3)$ & 109.0 & 110.3 & 108.8 & 107.6 \\
\hline 6 & & & 164.3 & 165.8 & 164.1 & 161.8 \\
\hline 7 & $6.42(\mathrm{~d}, J=2)$ & $6.39(\mathrm{~d}, J=1.3)$ & 106.8 & 107.1 & 106.6 & 109.6 \\
\hline 8 & & & 165.4 & 167.9 & 163.9 & 162.8 \\
\hline 9 & & & 107.7 & 108.3 & 108.0 & 108.7 \\
\hline 10 & & & 134.4 & 136.1 & 134.8 & 131.7 \\
\hline 11 & $\begin{array}{c}3.56(\mathrm{t}, J=6) \\
\text { calc: }{ }^{3} \mathrm{~J}_{11-12}=5.9 \\
{ }^{3} \mathrm{~J}_{11-16}=6.4\end{array}$ & $\begin{array}{c}3.57(\mathrm{~m}) \\
\text { calc: }{ }^{3} \mathrm{~J}_{11-16}=5.0\end{array}$ & 30.9 & 32.5 & 34.2 & 37.4 \\
\hline 12 & & & 126.0 & 126.3 & 131.7 & 131.1 \\
\hline 13 & & & 138.6 & 140.9 & 142.2 & 147.5 \\
\hline 14 & $\begin{array}{c}4.21(\mathrm{dd}, J=4,2) \\
\text { calc: }{ }^{3} \mathrm{~J}_{14-15}=2.4 \\
{ }^{3} \mathrm{~J}_{14-15}=3.2\end{array}$ & $\begin{array}{c}4.20(\mathrm{~m}) \\
\text { calc: }{ }^{3} \mathrm{~J}_{12-14}=3.7 \\
{ }^{3} \mathrm{~J}_{14-15}=7.0 \\
{ }^{3} \mathrm{~J}_{14-15}=9.5\end{array}$ & 64.0 & 63.8 & 62.3 & 67.9 \\
\hline 15 & $\begin{array}{l}1.40(\mathrm{dt}, J=4,13) \\
2.05(\mathrm{dt}, J=13,2)\end{array}$ & $\begin{array}{l}1.40(\mathrm{~m}) \\
2.05(\mathrm{~m})\end{array}$ & 28.5 & 30.4 & 30.2 & 28.0 \\
\hline 16 & $\begin{array}{c}2.19(\mathrm{ddd}, J=13,6 \\
2)\end{array}$ & $2.22(\mathrm{~m})$ & 34.4 & 35.9 & 35.5 & 42.5 \\
\hline 17 & & & 80.7 & 81.7 & 82.1 & 81.5 \\
\hline 18 & $1.36(\mathrm{~s})$ & $1.39, \mathrm{~s}$ & 24.7 & 25.1 & 23.3 & 23.7 \\
\hline 19 & $1.53(\mathrm{~s})$ & $1.55, \mathrm{~s}$ & 25.4 & 25.8 & 24.0 & 23.2 \\
\hline 20 & $\begin{array}{l}4.06(\mathrm{~d}, J=13) \\
4.16(\mathrm{~d}, J=13)\end{array}$ & $\begin{array}{l}4.13 \text { (d, no } J \\
\text { value given) }\end{array}$ & 65.6 & 64.7 & 64.2 & 68.7 \\
\hline $8-\mathrm{OH}$ & 12.01 & N. D. & & & & \\
\hline
\end{tabular}

${ }^{9}$ Wessels, P.; Gohrt, A.; Zeeck, A. J. Antibiot. 1991, 44, 1013.

${ }^{10}$ Li, X.; Xheng, Y.; Sattler, I.; Lin, W. Arch. Pharm. Res. 2006, $29,942$. 


\section{Molecular modelling and NMR calculations}

Calculations of molecular geometries and energies were carried out with density functional theory (DFT) using Gaussian 16 (revision A.03). ${ }^{11}$ Geometries optimisations, frequency analyses and single point energy calculations were run in the gas phase at the B3LYP/6-31G(d) level. Stable ground states were confirmed by the absence of imaginary vibrational frequencies. Calculations of NMR homonuclear ${ }^{1} \mathrm{H}$ spin-spin coupling constants were performed using the DU4 method. ${ }^{12}$ Selected vicinal Fermi contact (FC) values were scaled according to $J=1.196 * \mathrm{FC}-0.05$.

${ }^{13} \mathrm{C}$ NMR chemical shift calculations for naphthgeranine $\mathrm{C}(\mathbf{6})$ and naphthoquinone $\mathrm{C}(\mathbf{2 5})$ were run using the GIAO method at the mPW1PW91/6-311+G(2d,p) level in methanol solvent using the SMD continuum solvent model. ${ }^{13}$ Chemical shifts $(\boldsymbol{\delta})$ were calculated by scaling the isotropic magnetic shielding tensor values $(\boldsymbol{\sigma})$ according to $\boldsymbol{\delta}=($ intercept $-\boldsymbol{\sigma}) /($-slope $)$, where $\quad$ slope $=-1.0399$, intercept $=186.5993 .{ }^{14}$

Comparison of selected ${ }^{1} \mathrm{H}$ spin-spin coupling constants of compounds $\mathbf{1 9}$ and $\mathbf{2 0}$ with experimental data:
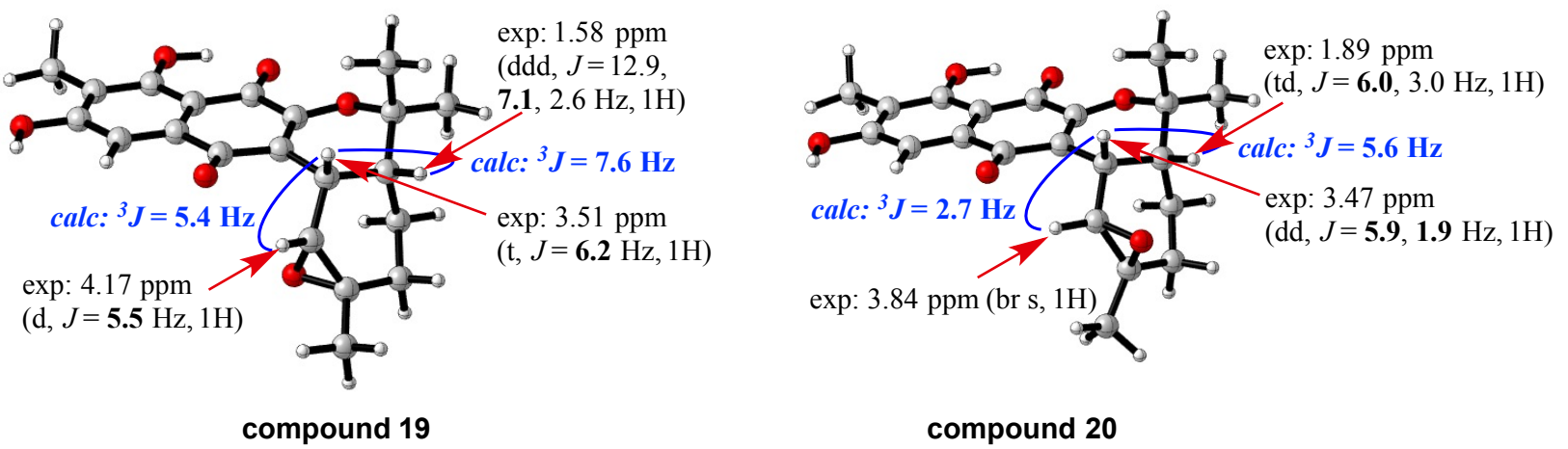

compound 20

\footnotetext{
${ }^{11}$ Frisch, M. J.; Trucks, G. W.; Schlegel, H. B.; Scuseria, G. E.; Robb, M. A.; Cheeseman, J. R.; Scalmani, G.; Barone, V.; Petersson, G. A.; Nakatsuji, H.; Li, X.; Caricato, M.; Marenich, A. V.; Bloino, J.; Janesko, B. G.; Gomperts, R.; Mennucci, B.; Hratchian, H. P.; Ortiz, J. V.; Izmaylov, A. F.; Sonnenberg, J. L.; Williams-Young, D.; Ding, F.; Lipparini, F.; Egidi, F.; Goings, J.; Peng, B.; Petrone, A.; Henderson, T.; Ranasinghe, D.; Zakrzewski, V. G.; Gao, J.; Rega, N.; Zheng, G.; Liang, W.; Hada, M.; Ehara, M.; Toyota, K.; Fukuda, R.; Hasegawa, J.; Ishida, M.; Nakajima, T.; Honda, Y.; Kitao, O.; Nakai, H.; Vreven, T.; Throssell, K.; Montgomery, J. A., Jr.; Peralta, J. E.; Ogliaro, F.; Bearpark, M. J.; Heyd, J. J.; Brothers, E. N.; Kudin, K. N.; Staroverov, V. N.; Keith, T. A.; Kobayashi, R.; Normand, J.; Raghavachari, K.; Rendell, A. P.; Burant, J. C.; Iyengar, S. S.; Tomasi, J.; Cossi, M.; Millam, J. M.; Klene, M.; Adamo, C.; Cammi, R.; Ochterski, J. W.; Martin, R. L.; Morokuma, K.; Farkas, O.; Foresman, J. B.; Fox, D. J. Gaussian, Inc., Wallingford CT, 2016.

12 Kutateladze, A. G.; Mukhina, O. A. J. Org. Chem. 2015, 80 (10), 5218-5225.

${ }^{13}$ Marenich, A. V.; Cramer, C. J.; Truhlar, D. G.; J. Phys. Chem. B 2009, 113, 6378.

${ }^{14}$ Pierens, G. K. J. Comput. Chem. 2014, 35 (18), 1388-1394.
} 
Molecular Structures and Energies

\section{Epoxide 19}

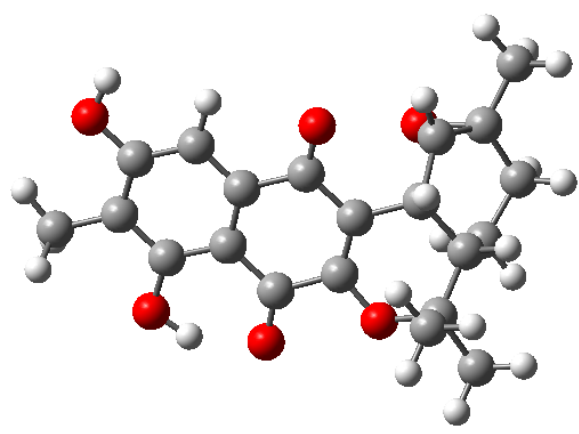

C $0.30505900-1.16563800 \quad 0.09326300$

$\begin{array}{llll}0 & 1.16643000 & -2.18025600 & -0.07223200\end{array}$

C $2.46234100-2.07147300 \quad 0.59901100$

C $3.28730900-3.20842800-0.00621000$

H $3.29803900-3.15918400-1.09688500$

H $2.85608100-4.17243300 \quad 0.28011300$

$\mathrm{H} \quad 4.31891800 \quad-3.16852800 \quad 0.36013700$

$\begin{array}{llll}\text { C } 2.24378700 & -2.33802800 & 2.09751300\end{array}$

$\mathrm{H} \quad 1.58174200-1.60152800 \quad 2.55957200$

H $3.20316800 \quad-2.31405600 \quad 2.62612200$

H $1.79870100 \quad-3.32787800 \quad 2.23640000$

C $3.05413000-0.66315300 \quad 0.34059300$

H $3.97546400-0.60050600 \quad 0.93741300$

C $3.44398300-0.41362500-1.12917600$

$\mathrm{H} 4.08108600-1.22369000-1.49528000$

H $2.54865900-0.39545200-1.75938200$

C $4.20595500 \quad 0.90716000-1.27926000$

H $5.18223200 \quad 0.83526700-0.77739700$

H $4.414339001 .10536000-2.33942300$

C $3.44731200 \quad 2.08242100-0.69558100$

C $2.41083800 \quad 1.82992300 \quad 0.32426700$

C $2.08193000 \quad 0.43237300 \quad 0.86674000$

H $2.19756900 \quad 0.51325000 \quad 1.95655500$

C $0.63682500 \quad 0.06345200 \quad 0.57357800$

o $2.04619200 \quad 2.10176900-1.04147000$

C $-1.08903300-1.52759200-0.33016400$

C $-0.42913400 \quad 1.02818700 \quad 0.89313700$

C $-1.82861100 \quad 0.70649400 \quad 0.47074900$

C $-4.49455000 \quad 0.13836200-0.31665400$

C $-2.12994200-0.53585600-0.12753300$

C $-2.82627400 \quad 1.63967500 \quad 0.67775000$

C $-4.14723100 \quad 1.35367200 \quad 0.28169700$

C $-3.46768500-0.80696000-0.51473700$

$\mathrm{H}-2.56927400 \quad 2.58690900 \quad 1.14444700$

$0-1.30322100-2.64244200-0.83383400$

$\begin{array}{llll}0 & -0.18784800 & 2.06518400 & 1.51213600\end{array}$

$\begin{array}{lllll}0 & -3.81015700 & -1.97358600 & -1.08124200\end{array}$

$\mathrm{H}-2.97971800-2.51715500-1.14109400$

$\begin{array}{llll}0 & -5.14128900 & 2.26584100 & 0.47378000\end{array}$

$\mathrm{H}-4.76419500 \quad 3.05842700 \quad 0.88690300$

C $-5.90168900-0.18456800-0.74291900$

$\mathrm{H}-6.27573800-1.06769700-0.21158500$

$\mathrm{H}-5.93705000-0.42556200-1.81155500$

$\mathrm{H}-6.57293200 \quad 0.65263900-0.54773400$

$\mathrm{H} 2.15042800 \quad 2.63757600 \quad 1.00368300$

C $4.13433600 \quad 3.42910500-0.76204200$

H $3.49544600 \quad 4.21066300-0.34068400$

$\mathrm{H} \quad 4.35706200 \quad 3.69396600-1.80285700$

$\mathrm{H} 5.081159003 .41452200-0.20870400$

Electronic Energy $(E E)=-1264.8172$ Hartree

Zero-point Energy Correction $=0.398147$ Hartree 


\section{Epoxide 20}

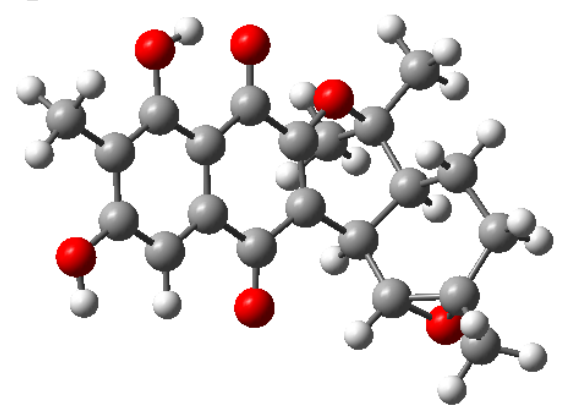

C $0.27343100-1.16350300 \quad 0.10381400$

$\begin{array}{llll}0 & 1.10974300 & -2.19841300 & -0.03757700\end{array}$

C $2.44869900-2.08178900 \quad 0.55057500$

C $3.23567000-3.22808600-0.08688200$

$\mathrm{H} 3.19876800-3.18699900-1.17720100$

H $2.81276800 \quad-4.18782700 \quad 0.22500300$

$\mathrm{H} \quad 4.28267200 \quad-3.19085400 \quad 0.23267000$

C $2.31759000-2.329139002 .06157900$

$\mathrm{H} 1.65047200-1.61076200 \quad 2.54405300$

$\begin{array}{llll}\mathrm{H} \quad 3.30055900 & -2.25665100 & 2.53987800\end{array}$

H $1.91921800 \quad-3.33238400 \quad 2.24038300$

C $3.01203200-0.67865600 \quad 0.22712300$

$\mathrm{H} \quad 3.98939100 \quad-0.59451400 \quad 0.71614100$

C $3.19760800-0.44014600-1.28547600$

H $3.78123500-1.25061000-1.73149800$

$\mathrm{H} \quad 2.21537100-0.46157900-1.77370200$

C $3.89379500 \quad 0.89126300-1.60486100$

$\mathrm{H} \quad 4.97659700 \quad 0.80425700-1.44364000$

$\mathrm{H} \quad 3.75634700 \quad 1.12094800 \quad-2.66876900$

C $3.400800002 .05861700-0.76236300$

$\begin{array}{lllll}0 & 3.93252600 & 2.05981600 & 0.58513400\end{array}$

C $2.527867001 .80837600 \quad 0.40165000$

H $1.88520000 \quad 2.61070800 \quad 0.75157800$

C $2.08563300 \quad 0.40968100 \quad 0.83430300$

H $2.20687400 \quad 0.41235300 \quad 1.92772200$

C $0.62798400 \quad 0.08557200 \quad 0.52884100$

C $3.39889500 \quad 3.41833600-1.42717200$

$\mathrm{H} 3.10238100 \quad 4.19550100-0.71678100$

H $2.70636000 \quad 3.43734400-2.27722600$

$\mathrm{H} 4.40018100 \quad 3.66242700-1.80348600$

C $-1.12925200-1.52986600-0.29585700$

C $-0.43632000 \quad 1.06206800 \quad 0.82626600$

C $-1.84433800 \quad 0.73004300 \quad 0.43603700$

C $-4.52556200 \quad 0.15157700 \quad-0.29419300$

C $-2.16239400-0.52870600-0.11512000$

C $-2.83433600 \quad 1.67539400 \quad 0.62617300$

C $-4.16296800 \quad 1.38295500 \quad 0.26122500$

C $-3.50693900-0.80577700 \quad-0.47463700$

$\mathrm{H}-2.56657400 \quad 2.63531500 \quad 1.05951100$

$\begin{array}{llll}0 & -1.35338700 & -2.65854500 & -0.76234200\end{array}$

$\begin{array}{llll}0 & -0.19776900 & 2.12805500 & 1.39715500\end{array}$

$0-3.86209400-1.98890500-0.99651000$

$\mathrm{H}-3.03518700-2.53841700-1.04973500$

$\begin{array}{llll}0 & -5.14990400 & 2.30465800 & 0.44068400\end{array}$

$\mathrm{H}-4.76492800 \quad 3.10698000 \quad 0.82697600$

C $-5.94102100-0.17619600-0.68785500$

$\mathrm{H}-6.34102000-0.98653000 \quad-0.06619900$

H $-5.98157400-0.52879400-1.72425600$

$\mathrm{H}-6.58928000 \quad 0.69465200 \quad-0.58278500$

Electronic Energy $(\mathrm{EE})=-\mathbf{1 2 6 4 . 8 1 5 8}$ Hartree Zero-point Energy Correction $=0.398173$ Hartree 


\section{Naphthgeranine C (6)}

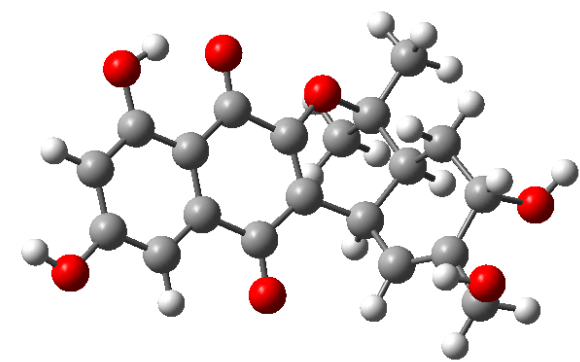

C $-0.29178700-1.27152000 \quad 0.02624500$

O $0.49832300-2.33241400-0.18088400$

C $1.76810000-2.38514100 \quad 0.55221500$

C $2.54971200-3.50177400-0.14095200$

$\mathrm{H} 2.63101400-3.32821700-1.21599600$

H $2.04011300-4.45899600 \quad 0.00507500$

$\mathrm{H} \quad 3.55725700 \quad-3.57711200 \quad 0.28197100$

C $1.45638800-2.79551100 \quad 1.99983600$

$\mathrm{H} 0.80470300 \quad-2.07723100 \quad 2.50374400$

$\mathrm{H} \quad 2.38582400 \quad-2.87396600 \quad 2.57441300$

H $0.95926600 \quad-3.77022300 \quad 2.01172300$

C $2.45171800-1.00239400 \quad 0.47131100$

H $3.36687300-1.05045500 \quad 1.07398300$

C $2.87212100-0.59953100-0.95291900$

H $3.47962200-1.38318700-1.41783500$

$\mathrm{H} 1.98843700-0.46180900-1.58943600$

C $3.69207100 \quad 0.69379100-0.93528200$

C $3.04174300 \quad 1.76284500-0.09348300$

C $2.06210800 \quad 1.48478600 \quad 0.77394300$

C $1.53796600 \quad 0.092104001 .08116100$

$\mathrm{H} \quad 1.55862700 \quad 0.00560400 \quad 2.17807700$

C $0.09065900 \quad-0.11491700 \quad 0.63982300$

C $-1.67243700-1.48486500-0.52420000$

C $-0.92930900 \quad 0.89203600 \quad 0.98117000$

C $-2.32062900 \quad 0.71685900 \quad 0.44211600$

C $-4.92212300 \quad 0.42314900-0.55691100$

C $-2.65561900-0.44316700 \quad-0.29494200$

C $-3.25644900 \quad 1.70508700 \quad 0.68090200$

C $-4.56278900 \quad 1.55208000 \quad 0.17352400$

C $-3.97569500-0.58404800 \quad-0.79576000$

$\mathrm{H}-2.98898100 \quad 2.58645900 \quad 1.25133800$

$\begin{array}{llll}0 & -1.92637200 & -2.52677300 & -1.15079000\end{array}$

$0-0.66747300 \quad 1.85242700 \quad 1.70502300$

o $-4.35942700-1.65842700-1.49519800$

$\mathrm{H}-3.56698500 \quad-2.25708200-1.55401000$

$\begin{array}{llll}0 & -5.43648700 & 2.55718200 & 0.43600800\end{array}$

$\mathrm{H}-6.29892500 \quad 2.34695500 \quad 0.04321500$

$\mathrm{H}-5.92702600 \quad 0.29379000-0.94977300$

C $3.56467400 \quad 3.16542200-0.28241900$

H $4.64843200 \quad 3.18073100-0.12925000$

$03.37323800 \quad 3.64129600-1.61945100$

$\mathrm{H} 2.41539000 \quad 3.65818600-1.77549700$

$\mathrm{H} \quad 1.60308700 \quad 2.28564100 \quad 1.34486000$

$\mathrm{H} \quad 3.10672600 \quad 3.84466900 \quad 0.45183700$

$05.00572500 \quad 0.47443900-0.38505100$

H $5.54478900 \quad 0.06185500-1.07812700$

$\mathrm{H} 3.789001001 .08080600-1.95982300$

Electronic Energy $(E E)=-1300.7072$ Hartree

Zero-point Energy Correction $=0.374891$ Hartree 


\section{Naphthoquinone C (25)}

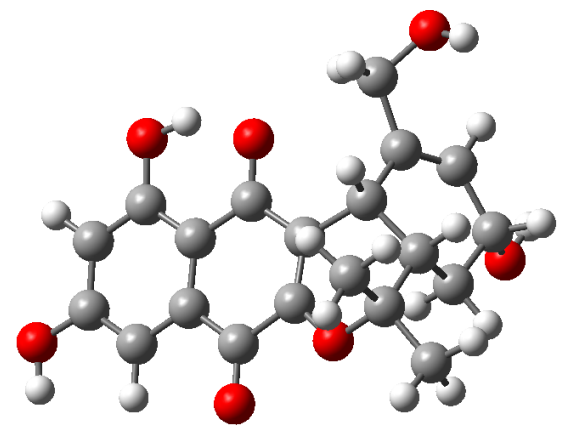

C $0.08793600-1.36794400-0.25377800$

$\begin{array}{lllll}0 & -0.74063400 & -2.41692900 & -0.30896000\end{array}$

C $-2.07860500-2.19830400-0.85666500$

C $-2.87525600-3.42674100-0.41324800$

$\mathrm{H}-2.84339500 \quad-3.56134200 \quad 0.66941700$

$\mathrm{H}-2.45524500-4.32594700 \quad-0.87383100$

$\mathrm{H}-3.92053000-3.33370700-0.72734200$

C $-1.96240400-2.18914900-2.38901500$

$\mathrm{H}-1.27943900-1.41588500-2.74931400$

$\mathrm{H}-2.94563600-2.01615600-2.84078400$

$\mathrm{H}-1.58847100-3.15620900-2.73811800$

C $-2.62010100-0.86669400-0.29015900$

$\mathrm{H}-3.61697500-0.71096800 \quad-0.72438400$

C $-2.74199100-0.87903100 \quad 1.24604400$

$\mathrm{H}-3.30450100-1.746753001 .59922900$

$\mathrm{H}-1.74878000 \quad-0.93499300 \quad 1.70343400$

C $-3.41508400 \quad 0.38578800 \quad 1.76594700$

C $-2.99237600 \quad 1.610106001 .00470100$

C $-2.24203000 \quad 1.62426300-0.10523100$

C $-1.71978100 \quad 0.32296300-0.73459900$

$\mathrm{H}-1.82538700 \quad 0.45962300-1.82074600$

C $-0.25751800-0.06319300-0.46838700$

C $1.47983400-1.79900500 \quad 0.13003000$

C $0.82342600 \quad 0.93023800-0.57186300$

C $2.19823800 \quad 0.57135700-0.20817300$

$\begin{array}{llll}\text { C } 4.82317600 & -0.11829400 & 0.52778000\end{array}$

C $2.52841400-0.74751400 \quad 0.16122100$

C $3.23301500 \quad 1.54527500-0.22631500$

C $4.53556400 \quad 1.19052700 \quad 0.15348800$

C $3.81850100-1.10179800 \quad 0.52610700$

$\begin{array}{llll}0 & 1.70962200 & -2.96694300 & 0.40181300\end{array}$

$0 \quad 0.569096002 .07948900-0.99254200$

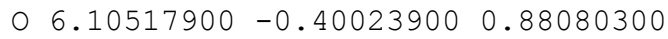

$\begin{array}{llll}0 & 3.01504800 & 2.81428700 & -0.59230800\end{array}$

C $-2.077649002 .94289000-0.85820700$

$\mathrm{H}-1.94458400 \quad 2.73575700-1.93175600$

$0-3.15115700 \quad 3.85335400-0.63910700$

$\mathrm{H}-3.97155700 \quad 3.39532100-0.88267900$

$\mathrm{H}-1.18262100 \quad 3.47297700-0.53408400$

$\mathrm{H}-3.40120900 \quad 2.55873400 \quad 1.35334500$

H $5.315734001 .94309500 \quad 0.14606200$

H $2.05557100 \quad 2.86968900 \quad-0.84727700$

$\mathrm{H} \quad 4.02675600-2.13351400 \quad 0.79537600$

H $6.17127600-1.33843800 \quad 1.11954000$

$\mathrm{H}-4.51045700 \quad 0.28299300 \quad 1.64732800$

$\begin{array}{lllll}0 & -3.10673600 & 0.47122900 & 3.16219500\end{array}$

$\mathrm{H}-3.50576200 \quad 1.28727800 \quad 3.50336000$

Electronic Energy (EE) $=-1300.6966$ Hartree

Zero-point Energy Correction $=0.374473$ Hartree 


\section{Single crystal X-ray data}

A single crystal of $\mathbf{1 9}$ was mounted in paratone-N oil on a Mitigen crystal mount and X-ray diffraction data were collected at 150(2) K on an Oxford X-calibur single crystal diffractometer using Mo Ka radiation. The data set was corrected for absorption using a multi-scan method, and the structures solved by direct methods using SHELXS-2008 and refined by full-matrix least squares on F2 by SHELXL-2015, ${ }^{15}$ interfaced through the programs X-Seed ${ }^{16}$ and Olex2 (Tables). ${ }^{17}$ Unless otherwise stated, non-hydrogen atoms were refined anisotropically and hydrogen atoms were included as invariants at geometrically estimated positions. Full data for the structure determination have been deposited with the Cambridge Crystallographic Data Centre as CCDC 1946257. Copies of this information may be obtained free of charge from The Director, CCDC, 12 Union Street, Cambridge CB2 1EZ, U.K. (fax, +44-1223-336-033; e-mail, deposit@ccdc.cam.ac.uk). The table below provides the crystal data and structure refinement details for compound $\mathbf{1 9 .}$

Compound 19 crystallises in the triclinic space group $P-1$ with four complete molecules and four dichloroethane solvate molecules in the asymmetric unit; these comprise two crystallographically independent pairs of hydrogen bonded dimers of 19 in which each dimer comprises a pair of molecules of the same stereochemistry (the dimers of 19 within the asymmetric unit are enantiomers consistent with the compound being a racemic mixture). The figures below show a representation of the asymmetric unit and one selected molecule (thermal ellipsoids shown at the $50 \%$ probability level), a view of one of the hydrogen bonded dimers with the intramolecular and intermolecular hydrogen bonds shown and finally a packing diagram of the structure viewed down the $a$-axis.

15 (a) Sheldrick, G., SHELXS-2014 and SHELXL-2014. Program for X-ray Crystal Structure Determination, Göttingen University, Göttingen, Germany 2014. (b) Sheldrick, G. M. A short history of SHELX Acta Crystallographica Section A: Foundations of Crystallography 2008, 64, 112. (c) Sheldrick, G. M. Crystal structure refinement with SHELXL Acta Crystallographica Section C: Structural Chemistry 2015, 71, 3.

${ }_{16}$ Barbour, L. J. X-Seed - A Software Tool for Supramolecular Crystallography Journal of Supramolecular Chemistry 2001, 1, 189-191.

${ }^{17}$ Dolomanov, O. V.; Bourhis, L. J.; Gildea, R. J.; Howard, J. A.; Puschmann, H. OLEX2: a complete structure solution, refinement and analysis program Journal of Applied Crystallography 2009, 42, 339. 


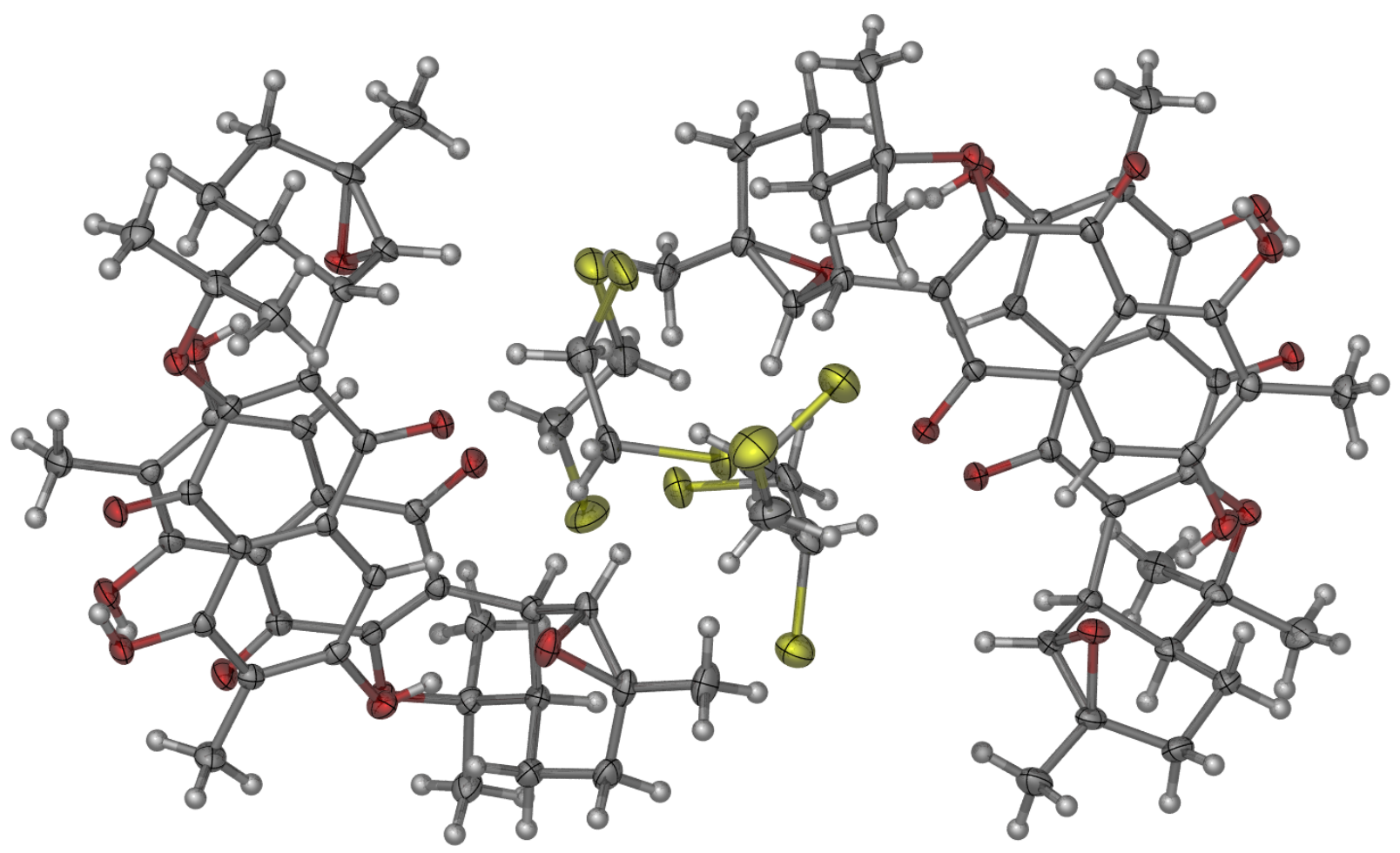

(a)

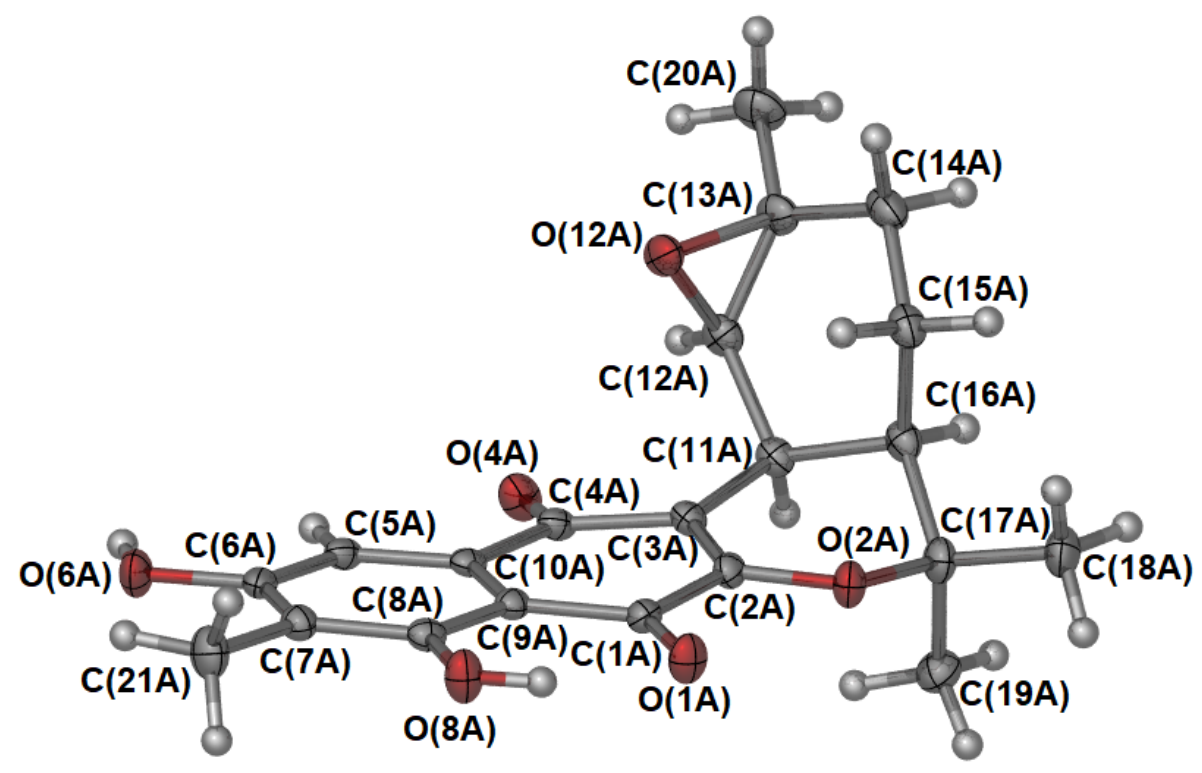

(b)

Figure S6.1. (a) A perspective view of the asymmetric unit of 19 with thermal ellipsoids shown at the $50 \%$ probability level. Carbon - grey; hydrogen - white; oxygen - red; chlorine - yellow. (b) One selected molecule of $\mathbf{1 9}$ is shown to more clearly represent the molecular structure. 


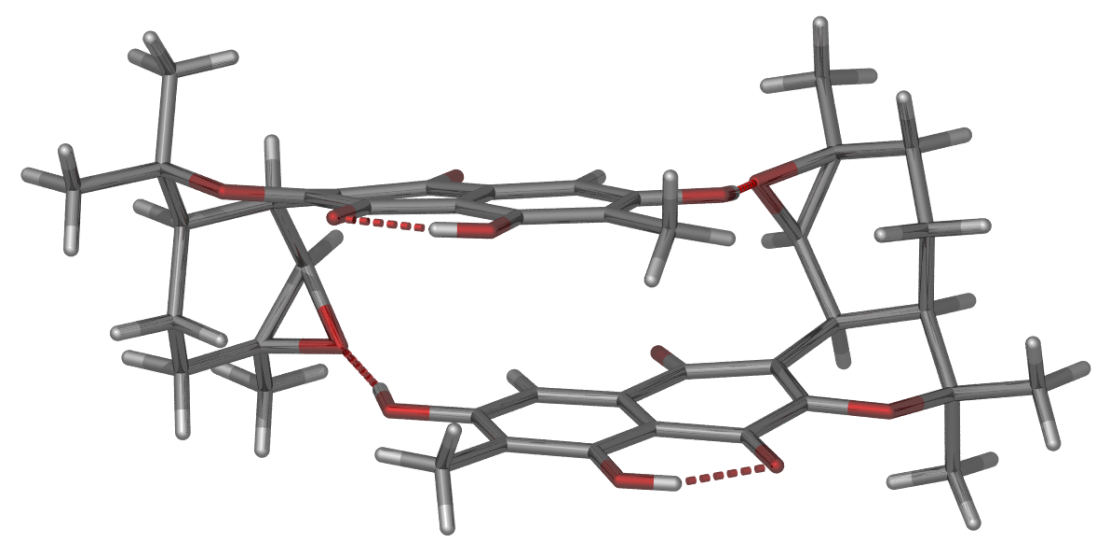

Figure S6.2. A perspective view of one of the hydrogen bonded dimers within the structure of 19. The hydrogen bonds are shown with the dashed lines.

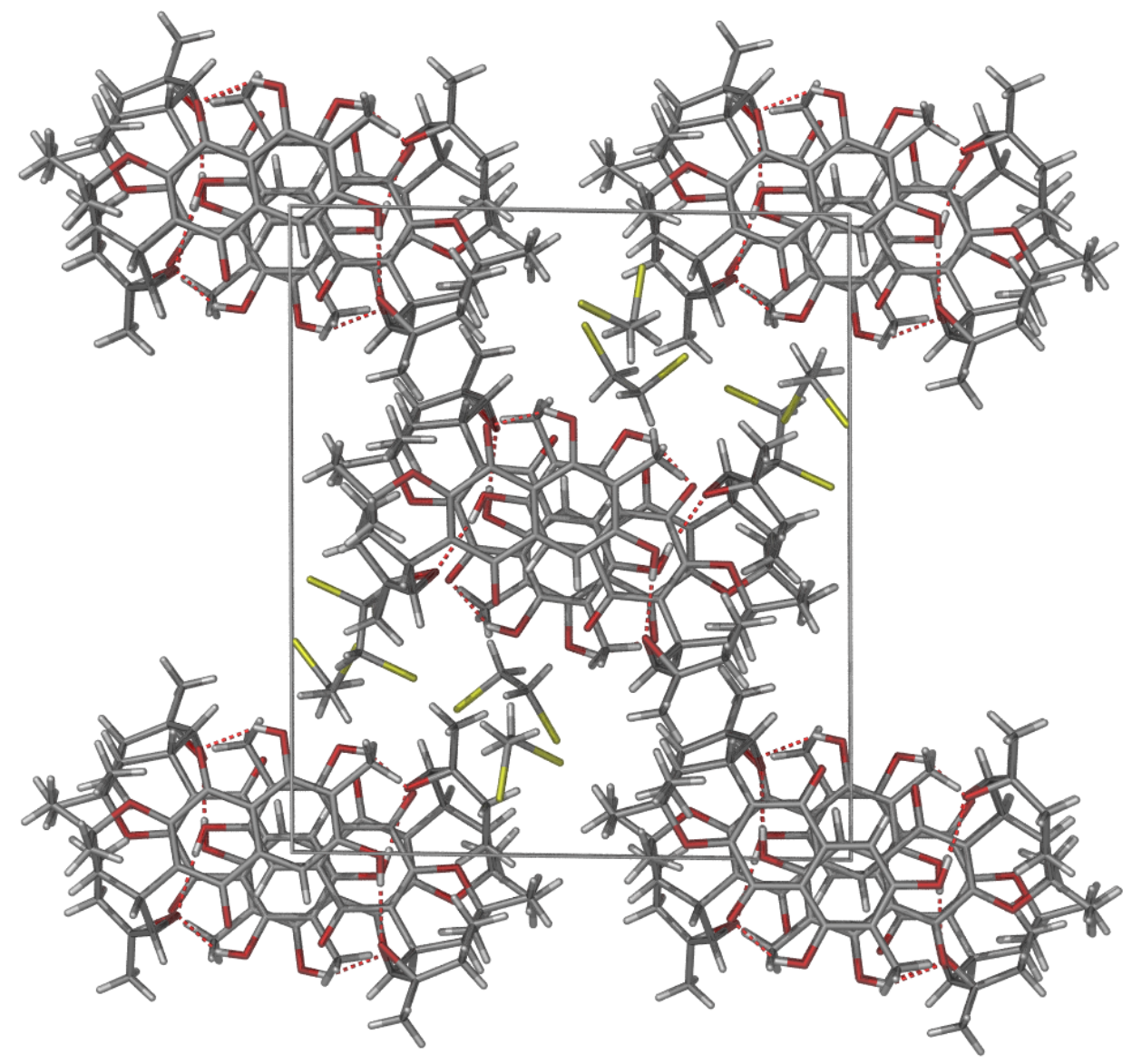

Figure S6.3. A view down the $a$-axis of the packing of compound 19 . 
Table 6.1. Crystal data and structure refinement for 19.

\begin{tabular}{|c|c|}
\hline Identification code & LMX1 \\
\hline CCDC number & 1946257 \\
\hline Empirical formula & $\mathrm{C}_{92} \mathrm{H}_{104} \mathrm{NO}_{24} \mathrm{Cl}_{8}$ \\
\hline Formula weight & 1877.35 \\
\hline Temperature/K & $150.1(2)$ \\
\hline Crystal system & triclinic \\
\hline Space group & $\mathrm{P}-1$ \\
\hline $\mathrm{a} / \AA$ & $13.4564(3)$ \\
\hline $\mathrm{b} / \AA$ & $17.9080(3)$ \\
\hline $\mathrm{c} / \AA$ & $19.7291(3)$ \\
\hline$\alpha /^{\circ}$ & $85.6940(10)$ \\
\hline$\beta /^{\circ}$ & $80.149(2)$ \\
\hline$\gamma /{ }^{\circ}$ & $70.114(2)$ \\
\hline Volume $/ \AA^{3}$ & $4404.13(15)$ \\
\hline $\mathrm{Z}$ & 2 \\
\hline$\rho_{\text {calc }} \mathrm{g} / \mathrm{cm}^{3}$ & 1.416 \\
\hline$\mu / \mathrm{mm}^{-1}$ & 0.333 \\
\hline $\mathrm{F}(000)$ & 1968.0 \\
\hline Crystal size $/ \mathrm{mm}^{3}$ & $0.72 \times 0.22 \times 0.12$ \\
\hline Radiation & $\operatorname{MoK} \alpha(\lambda=0.71073)$ \\
\hline $2 \theta$ range for data collection $/{ }^{\circ}$ & 6.698 to 58.926 \\
\hline Index ranges & $-18 \leq \mathrm{h} \leq 18,-24 \leq \mathrm{k} \leq 24,-27 \leq 1 \leq 25$ \\
\hline Reflections collected & 97503 \\
\hline Independent reflections & $21565\left[\mathrm{R}_{\text {int }}=0.0435, \mathrm{R}_{\text {sigma }}=0.0395\right]$ \\
\hline Data/restraints/parameters & $21565 / 0 / 1141$ \\
\hline Goodness-of-fit on $\mathrm{F}^{2}$ & 1.018 \\
\hline Final $\mathrm{R}$ indexes $[\mathrm{I}>=2 \sigma(\mathrm{I})]$ & $\mathrm{R}_{1}=0.0472, \mathrm{wR}_{2}=0.1064$ \\
\hline Final R indexes [all data] & $\mathrm{R}_{1}=0.0766, \mathrm{wR}_{2}=0.1220$ \\
\hline Largest diff. peak/hole / e $\AA^{-3}$ & $0.65 /-0.54$ \\
\hline
\end{tabular}

